\title{
SIMPLY CONNECTED SURFACES OF GENERAL TYPE IN POSITIVE CHARACTERISTIC VIA DEFORMATION THEORY
}

\author{
YONGNAM LEE AND NOBORU NAKAYAMA
}

\begin{abstract}
Algebraically simply connected surfaces of general type with $p_{g}=q=0$ and $1 \leq K^{2} \leq 4$ in positive characteristic (with one exception in $K^{2}=4$ ) are presented by using a $\mathbb{Q}$-Gorenstein smoothing of two-dimensional toric singularities, a generalization of Lee-Park's construction [33] to the positive characteristic case, and Grothendieck's specialization theorem for the fundamental group.
\end{abstract}

\section{Contents}

1. Introduction

2. Linear chains of rational curves as exceptional loci

3. Toric singularity of class $\mathrm{T}$

4. Review of deformation theory

5. Deformations of certain projective surfaces with toric singularities of class $\mathrm{T}$

6. Simply connected surfaces of general type with $p_{g}=q=0$

7. Proof of Main Theorem

References

\section{INTRODUCTION}

One of the interesting problems in the classification of algebraic surfaces is to find a new family of simply connected surfaces of general type with geometric genus $p_{g}=0$. Surfaces with $p_{g}=0$ are interesting in view of Castelnuovo's criterion: An irrational surface with irregularity $q=0$ must have bigenus $P_{2} \geq 1$. Simply connected surfaces of general type with $p_{g}=0$ are little known and the classification is still open.

2000 Mathematics Subject Classification. Primary 14J29; Secondary 14B07, 14J17.

Key words and phrases. surface of general type, $\mathbb{Q}$-Gorenstein smoothing, two-dimensional toric singularity, algebraic fundamental group.

The first named author is partly supported by the WCU Grant funded by the Korean Government (R33-2008-000-10101-0), and by the National Research Foundation of Korea(NRF) grant funded by the Korea government(MEST) (No. 2010-0008752). The second named author is partly supported by the Grant-in-Aid for Scientific Research (C), Japan Society for the Promotion of Science. 
When a surface is defined over the field $\mathbb{C}$ of complex numbers, the only known simply connected minimal surfaces of general type with $p_{g}=0$ were Barlow surfaces [7] until 2006. The canonical divisor of Barlow surfaces satisfies $K^{2}=1$. Recently, the first named author and J. Park [33] have constructed a simply connected minimal surface of general type with $p_{g}=0$ and $K^{2}=2$ by using a $\mathbb{Q}$-Gorenstein smoothing and Milnor fiber of a smoothing (or rational blow-down surgery). When a surface is defined over a field of positive characteristic, the existence of algebraically simply connected minimal surface of general type with $p_{g}=0$ is known only for some special characteristics. Lang [28] has constructed surfaces of general type with $p_{g}=0$ and $K^{2}=1$ in characteristic 5. Ekedahl [13] has given examples of surfaces of general type with $p_{g}=0$ and with $1 \leq K^{2} \leq 9$ in characteristic two. The inequality $1 \leq K_{S}^{2} \leq 9$ holds by results of Shepherd-Barron [50], [51] when $S$ is a minimal surface of general type with $p_{g}=0$. This is shown in Liedtke's lecture notes [35] on algebraic surfaces in positive characteristic.

We shall construct such a surface of general type defined over an algebraically closed field of any characteristic applying the Lee-Park construction given in [33]. The following is our main result:

Main Theorem. For any algebraically closed field $\mathbb{k}$ and for any integer $1 \leq K^{2} \leq 4$, there exists an algebraically simply connected minimal surface $\mathbb{S}$ of general type over $\mathbb{k}$ with $p_{g}(\mathbb{S})=q(\mathbb{S})=\operatorname{dim} \mathrm{H}^{2}\left(\mathbb{S}, \Theta_{\mathbb{S} / \mathbb{k}}\right)=0$ and $K_{\mathbb{S}}^{2}=K^{2}$ except $\left(\operatorname{char}(\mathbb{k}), K^{2}\right)=(2,4)$, where $\Theta_{\mathbb{S} / \mathbb{k}}$ denotes the tangent sheaf. Moreover, one can find such a surface with ample canonical divisor when $1 \leq K^{2} \leq 4$, except $\left(\operatorname{char}(\mathbb{k}), K^{2}\right)=(2,1),(2,2)$, and $(2,4)$.

Remark. (1) The surface $\mathbb{S}$ in Main Theorem is liftable to characteristic zero since $\mathrm{H}^{2}\left(\mathbb{S}, \Theta_{\mathbb{S} / \mathbb{k}}\right)=\mathrm{H}^{2}\left(\mathbb{S}, \mathcal{O}_{\mathbb{S}}\right)=0$.

(2) In our method of constructing $\mathbb{S}$, the bound $K^{2} \leq 4$ is necessary (cf. Remark 6.1 below).

(3) The ampleness property in Main Theorem is known in characteristic zero by 44, Section 2] and the proof of [43, Theorem 4.1] in the case of $K^{2}=3$ and 4 .

(4) We have the exceptional cases of $\left(\operatorname{char}(\mathbb{k}), K^{2}\right)$ by the lack of certain examples of rational surfaces $X$ in Section 6. This is a technical reason, and the existence of such a surface $\mathbb{S}$ in the exceptional cases is an open problem.

The Lee-Park construction is as follows in the case of $K^{2}=2$ (cf. [33, Section 3]): First, we consider a special pencil of cubics in $\mathbb{P}^{2}$ and blow up many times to get a projective surface $M(\tilde{Z}$ in [33]) which contains a disjoint union of five linear chains of smooth rational curves representing the resolution graphs of special quotient singularities called of class T (cf. Definition 3.2 below). Then, we contract these linear chains of rational curves from the surface $M$ to produce a projective surface $X$ with five special 
quotient singularities of class $\mathrm{T}$ and with $K_{X}^{2}=2$. We can prove the existence of a global $\mathbb{Q}$-Gorenstein smoothing of the singular surface $X$ (cf. [33, Theorem 4]), in which a general fiber $X_{t}$ of the $\mathbb{Q}$-Gorenstein smoothing is a simply connected minimal surface of general type with $p_{g}=0$ and $K^{2}=2$ (cf. [33, Proposition 8]). The method of Lee-Park construction works to other types of rational elliptic surfaces, which are used to construct a simply connected minimal surface of general type with $p_{g}=0$ and with $K^{2}=1$, 3 , or 4 ([33, Section 7], [42], [43]). We shall show that the Lee-Park construction of singular surfaces also works in positive characteristic, but several key parts in the proof to show the existence of a global $\mathbb{Q}$-Gorenstein smoothing should be modified.

Over the field $\mathbb{C}$ of complex numbers, the existence of a local $\mathbb{Q}$-Gorenstein smoothing of a singularity of class $\mathrm{T}$ is given by the index-one cover (cf. [37, Proposition 5.9], [26, Proposition 3.10]). The key idea in [33] to show the vanishing of the obstruction space for a global $\mathbb{Q}$-Gorenstein smoothing is to use the lifting property of derivations of normal surface to its minimal resolutions, the tautness of the quotient singularities, and the special configurations of resolution graphs of singular points.

In characteristic 0, the tautness holds for quotient singularities (cf. [9, Satz 2.10]), i.e, the minimal resolution graph of a quotient singularity determines the type of singularity. It is known in characteristic 0 that the tautness is equivalent to $H^{1}\left(\Theta_{D}\right)=0$ for any "sufficiently large" effective divisor $D$ supported on the exceptional divisors on its minimal resolution (cf. [30, Theorem 3.10], [31, Section 2]). However, tautness does not hold in characteristic $p>0$ in general. Indeed, we have some examples of rational double points when $p=2,3$, and 5, by Artin's classification in [6]. The lifting property of derivations from a normal surface to its minimal resolution exists in characteristic 0 ([10, Proposition 1.2]), but this is not true in characteristic $p>0$ (cf. [5, Example, pp. 345] and Proposition 2.11,(4) below). In [53], the proof of Theorem $\mathrm{C}$ illustrates why the lifting property is guaranteed to hold only in characteristic 0 , and also provides sufficient conditions for its truth in characteristic $p>0$.

However, in our constructions, we have only two-dimensional toric singularities by contracting linear chains of smooth rational curves. In Section 2 below, we prove that the singularity obtained by contracting a linear chain of smooth rational curves is a two-dimensional toric singularity and it is taut (cf. Theorem 2.7 below). Moreover, it turns out that the lifting property of derivations mentioned above is not so important for proving the vanishing of the obstruction space (cf. Corollary 2.12 below).

In Section 3, we introduce the notion of toric surface singularity of class T (cf. Definition 3.2), explain some properties with Tables 1 and 2 of related numbers for some special cases, and construct a so-called $\mathbb{Q}$-Gorenstein smoothing explicitly by using toric description in Theorem 3.8 , 
We recall several basics on the deformation theory of schemes in Section 4 including Schlessinger's theory of functors on Artinian rings. As an exercise, using cotangent complexes and obstruction theory, we shall prove in Theorem 4.6 that if $\mathrm{H}^{2}\left(X, \Theta_{X / \mathbb{k}}\right)=0$ for an algebraic $\mathbb{k}$-variety $X$ with only isolated singularities, then the morphism $\operatorname{Def}_{X} \rightarrow \operatorname{Def}_{X}^{(\text {loc })}$ between the global and local the deformation functors of $X$ is smooth in the sense of Schlessinger (cf. [55, Proposition 6.4]). An algebraization result (Theorem 4.7) is added, which plays an important role when we construct an algebraic deformation. The proof uses Artin's theory of algebraization (cf. [3]).

In Section 5, we shall construct a deformation of a normal projective surface $X$ with toric singularities of class $\mathrm{T}$ assuming some extra conditions. As a consequence, we have a so-called $\mathbb{Q}$-Gorenstein smoothing not only over the base field $\mathbb{k}$ but also over a complete discrete valuation ring with the residue field $\mathbb{k}$ (cf. Theorems 5.2 and 5.4). As a byproduct, in Corollary 5.3, we can give a correct version of the existence of $\mathbb{Q}$-Gorenstein smoothing of any log del Pezzo surfaces of index two in positive characteristic (cf. [40, Theorem 5.16]). By the smoothing over the discrete valuation ring and the Grothendieck specialization theorem (cf. [SGA1, Exp. X, Corollaire 2.4, Théorème 3.8]), the algebraic simply connectedness of the smooth fiber is reduced to that of a smooth fiber of a $\mathbb{Q}$ Gorenstein smoothing of a reduction of $X$ of our construction to the complex number field $\mathbb{C}$. Note that the simply connectedness in case $\mathbb{k}=\mathbb{C}$ for the known Lee-Park constructions has been proved in [33], [42], and [43] by using Milnor fiber (or rational blow-down) and by applying van-Kampen's theorem on the minimal resolution of $X$.

Our plan of the proof of the Main Theorem is as follows: We first construct a normal rational surface $X$ with only toric singularities of class $\mathrm{T}$ satisfying extra conditions by Lee-Park's method. This $X$ is constructed from a suitable cubic pencil $\Phi$ on $\mathbb{P}^{2}$, a special construction of a birational morphism $M \rightarrow \mathbb{P}^{2}$, and a blowdown $M \rightarrow X$ of a union of linear chains of rational curves defining toric singularities of class T. Second, we apply the results in Section 5 to $X$ and obtain a so-called $\mathbb{Q}$-Gorenstein smoothing of $X$, in which a general smooth fiber $X_{t}$ is an expected surface satisfying the required conditions in Main Theorem.

In Section 6, we shall explain the outline of our proof giving sufficient conditions for the data needed in constructing $X$. During the discussion, from the conditions, we shall prove some key results such as the vanishing $\mathrm{H}^{2}\left(X, \Theta_{X / \mathbb{k}}\right)=0$, and the nef and bigness of $K_{X}$.

In Section 7, we shall give eight examples working in our proof. Most examples are taken from [33], 42], and [43], but Examples 7.6 7.8 are new which are presented by Heesang Park. These new examples are needed because of some problems in small characteristics. For example, rational surfaces admitting a minimal elliptic fibration with a 
configuration type $\left(\mathrm{I}_{8}, \mathrm{I}_{1}, \mathrm{I}_{1}, \mathrm{I}_{2}\right)$ of singular fibers are used in [42] and [43], but in characteristic 2, such a configuration does not exist [29]. Moreover, the vanishing $\mathrm{H}^{2}\left(X, \Theta_{X / \mathbb{k}}\right)=0$ does not hold in small characteristic in some cases. By checking the conditions of Section 6 for all the examples, we finally complete the proof of the Main Theorem.

Acknowledgements. The first named author would like to thank Research Institute for Mathematical Sciences (RIMS) in Kyoto University and Korea Institute for Advanced Study for their hospitality during his visit. Main part of the paper was worked out during his stay at RIMS in 2010. The authors express their gratitude to Professors Jonathan Wahl and Christian Liedtke for the invaluable comments. The authors are grateful to Dr. Takuzo Okada and Dr. Hisanori Ohashi for their careful reading of the draft versions, to Dr. Heesang Park for her providing Examples 7.6 17.8, and to Prof. Dongsoo Shin for his drawing figures. The authors thank to the referee for suggesting many improvements.

Notation and conventions. In this article, we fix an algebraically closed field $\mathbb{k}$ of characteristic $p \geq 0$.

- An algebraic scheme over a field $\mathbb{K}$ means a $\mathbb{K}$-scheme of finite type. This is called also an algebraic $\mathbb{K}$-scheme. An algebraic variety over a field $\mathbb{K}$ (or an algebraic $\mathbb{K}$-variety) is an integral separated algebraic scheme over $\mathbb{K}$.

- For an algebraic $\mathbb{k}$-scheme $X, \Omega_{X / \mathbb{k}}^{1}$ denotes the sheaf of one-forms and $\Theta_{X / \mathbb{k}}$ denotes the tangent sheaf, i.e., $\Theta_{X / \mathbb{k}}=\mathcal{H} o m_{\mathcal{O}_{X}}\left(\Omega_{X / \mathbb{k}}^{1}, \mathcal{O}_{X}\right)$. For a non-singular algebraic $\mathbb{k}$-variety $X$ and a normal crossing divisor $B, \Omega_{X / \mathbb{k}}^{1}(\log B)$ denotes the sheaf of logarithmic one-forms with poles along only $B$. Its dual $\mathcal{H} o m_{\mathcal{O}_{X}}\left(\Omega_{X / \mathbb{k}}^{1}(\log B), \mathcal{O}_{X}\right)$ is denoted by $\Theta_{X / \mathbb{k}}(-\log B)$, which is identified with the sheaf of derivations $\delta \in \Theta_{X / \mathbb{k}}$ such that $\delta\left(\mathcal{O}_{X}(-B)\right) \subset \mathcal{O}_{X}(-B)$.

- For a normal integral separated scheme $X$ and for a (Weil) divisor $D$, the reflexive sheaf $\mathcal{O}_{X}(D)$ of rank one is by definition the subsheaf of the sheaf of rational functions determined by the following property: A non-zero rational function $\varphi$ is contained in $\mathrm{H}^{0}\left(\mathcal{U}, \mathcal{O}_{X}(D)\right)$ for a non-empty open subset $\mathcal{U}$ if and only if $\left.\operatorname{div}(\varphi)\right|_{\mathcal{U}}+\left.D\right|_{\mathcal{U}} \geq 0$ for the principal divisor $\left.\operatorname{div}(\varphi)\right|_{\mathcal{U}}$ on $\mathcal{U}$.

- The maximal ideal of a local ring $A$ is denoted by $\mathfrak{m}_{A}$.

- A geometric point $t$ of a scheme $X$ is by definition a morphism $t: \operatorname{Spec} \mathbb{k}(t) \rightarrow X$ for an algebraically closed field $\mathbb{k}(t)$. For any scheme $Z$ over $X$, the geometric fiber $Z_{t}$ over the geometric point $t$ is defined to be the fiber product $Z \times_{X, t} \operatorname{Spec} \mathbb{k}(t)$.

- An étale neighborhood of a pair $(X, x)$ of a scheme $X$ and a point $x$ is by definition a pair $\left(X^{\prime}, x^{\prime}\right)$ of a scheme $X^{\prime}$ étale over $X$ and a point $x^{\prime}$ lying over $x$ such that the induced homomorphism between the residue fields at $x$ and $x^{\prime}$ is isomorphic (cf. [2, Section 2]). 
- In this article, the (étale) fundamental group of a scheme $X$ is called the algebraic fundamental group and is denoted by $\pi_{1}^{\text {alg }}(X)$ to avoid confusion with the topological fundamental group of a complex algebraic scheme (cf. [SGA1, Exp. XII, Corollaire 5.2]).

\section{Linear Chains of RATiOnal CURVES AS EXCEPTIONAL LOCI}

The main purpose of this section is to prove that any two-dimensional singularity having a linear chain of smooth rational curves as the exceptional locus of the minimal resolution is "toric." In other words, we prove the tautness (cf. [31]) of such singularities. In the case of characteristic zero, the singularity is just the cyclic quotient singularity of type $\frac{1}{n}(1, q)$ for some integers $n>q>0$ with $\operatorname{gcd}(n, q)=1$, and the tautness is known by [9, Satz 2.10].

In this section, let us fix a Noetherian ring $\Lambda$ and positive integers $n, q$ such that $n>q$ and $\operatorname{gcd}(n, q)=1$. We define integers $b_{1}, \ldots, b_{l}$ greater than one by the continued fraction:

$$
n / q=\left[b_{1}, \ldots, b_{l}\right]:=b_{1}-\frac{1}{b_{2}-\frac{1}{\ddots \cdot-\frac{1}{b_{l}}}}
$$

and introduce the following two conditions:

Definition 2.1 (Conditions $C(n, q)$ and $\left.C(n, q)^{\prime}\right)$. Let $Y$ be an affine algebraic flat $\Lambda$ scheme and let $\Sigma$ be a closed subscheme such that

- every geometric fiber of $Y \rightarrow \operatorname{Spec} \Lambda$ is a normal surface,

- $\Sigma$ is a section of $Y \rightarrow \operatorname{Spec} \Lambda$, and

- $Y \backslash \Sigma$ is smooth over $\operatorname{Spec} \Lambda$.

The pair $(Y, \Sigma)$ is said to satisfy condition $C(n, q)$ with a proper surjective morphism $\mu: M \rightarrow Y$ if the following conditions are satisfied:

(1) $M \rightarrow Y \rightarrow \operatorname{Spec} \Lambda$ is smooth and $\mu$ is an isomorphism over $Y \backslash \Sigma$.

(2) $\mu^{-1}(\Sigma)$ is a divisor $\sum_{i=1}^{l} E_{i}$ such that:

(a) Any $E_{i}$ is a Cartier divisor with $E_{i} \simeq \mathbb{P}_{\Lambda}^{1}$ and $\mathcal{O}_{E_{i}}\left(-E_{i}\right) \simeq \mathcal{O}\left(b_{i}\right)$.

(b) $E_{i} \cap E_{j}=\emptyset$ if $|i-j|>1$.

(c) The scheme-theoretic intersection $\Sigma_{i}:=E_{i-1} \cap E_{i}$ is a section over $\operatorname{Spec} \Lambda$ for all $2 \leq i \leq l$.

If, in addition, there exist divisors $B_{1}, B_{2}$ on $Y$ satisfying the conditions below, then $\left(Y, B_{1}, B_{2}\right)$ is said to satisfy condition $C(n, q)^{\prime}$ :

(3) The set-theoretic intersection $B_{1} \cap B_{2}$ is $\Sigma$. 
(4) There is a relative Cartier divisor $E_{0}\left(\right.$ resp. $\left.E_{l+1}\right)$ with respect to $M \rightarrow \operatorname{Spec} \Lambda$ such that:

(a) $E_{0} \cap E_{i}=\emptyset$ and $E_{j} \cap E_{l+1}=\emptyset$ for all $i>1$ and $j<l$.

(b) The scheme-theoretic intersections $\Sigma_{1}:=E_{0} \cap E_{1}$ and $\Sigma_{l+1}:=E_{l} \cap E_{l+1}$ are sections of $M \rightarrow \operatorname{Spec} \Lambda$.

(c) $B_{1}\left(\right.$ resp. $\left.B_{2}\right)$ is the image of $E_{l+1}$ (resp. $\left.E_{0}\right)$ by $\mu: M \rightarrow Y$.

Remark 2.1.1. In the situation above, the "dual graph" of $\sum_{i=0}^{l+1} E_{i}$ is written as

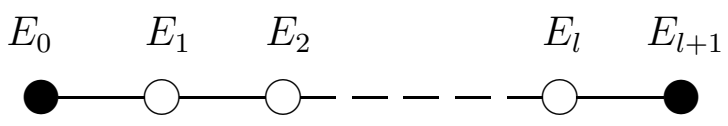

where the ends are distinguished, since these are not $\mu$-exceptional.

Remark 2.1.2. If $\Lambda$ is an algebraically closed field, then the condition $C(n, q)$ means that $Y$ is a normal affine surface with a singular point $\Sigma$ in which the exceptional locus of the minimal resolution is a linear chain of smooth rational curves. Moreover, in this case the condition $C(n, q)^{\prime}$ means that $B_{1}$ and $B_{2}$ are prime divisors on $Y$ with $B_{1} \cap B_{2}=\Sigma$ in which the dual graph of $\mu^{-1}\left(B_{1} \cup B_{2}\right)$ is also a linear chain and the end components correspond to the proper transforms of $B_{1}$ and $B_{2}$.

In this section we will do the following:

- Giving an explicit construction of a toric $\Lambda$-scheme $V$ with two boundary divisors $D_{1}, D_{2}$ such that $\left(V, D_{1}, D_{2}\right)$ satisfies $C(n, q)^{\prime}$.

- Showing that any $(Y, \Sigma)$ satisfying $C(n, q)$ is "étale equivalent to" $V$ along $\Sigma$ when $\Lambda$ is a field (cf. Theorem 2.6) ).

- Showing that any $\left(Y, B_{1}, B_{2}\right)$ satisfying $C(n, q)^{\prime}$ is "étale over" $\left(V, D_{1}, D_{2}\right)$ when $\Lambda$ is a local ring (cf. Theorem 2.7).

- As applications of Theorems 2.6] and2.7, giving some local properties of the surface singularity which has a linear chain of smooth rational curves as the exceptional locus of the minimal resolution.

Remark. When $\Lambda$ is an algebraically closed field, Theorem 2.6 corresponds to the "tautness" (cf. [31]) for two-dimensional normal singularities having linear chains of smooth rational curves as the exceptional locus of the minimal resolution. In positive characteristic, the tautness for linear chains of smooth rational curves seems to be well-known, but the authors could not find any reference. Recently, Hara has found another proof of Theorem 2.6 (cf. Case (1) in the proof of [17, Theorem 2.1]) using an argument in [8].

We begin with constructing $V$ by applying the theory of toric varieties or of torus embeddings. We refers the reader to [12, [24], [11], 411, etc. for more details of the theory. 
Let $\mathrm{N}_{0}$ be a free abelian group of rank two with a basis $\left(e_{1}, e_{2}\right)$, i.e., $\mathrm{N}_{0}=\mathbb{Z} e_{1}+\mathbb{Z} e_{2}$. For the fixed integers $n$ and $q$ above, we set

$$
v:=(1 / n)\left(e_{1}+q e_{2}\right) \in \mathrm{N}_{0, \mathbb{Q}}=\mathrm{N}_{0} \otimes \mathbb{Q} \quad \text { and } \quad \mathrm{N}:=\mathrm{N}_{0}+\mathbb{Z} v \subset \mathrm{N}_{0, \mathbb{Q}} .
$$

Now, we define $V$ to be the affine toric $\Lambda$-scheme $\mathbb{T}_{\mathrm{N}}(\boldsymbol{\sigma})$ associated with $\mathrm{N}$ and with the cone $\boldsymbol{\sigma}=\mathbb{R}_{\geq 0} e_{1}+\mathbb{R}_{\geq 0} e_{2} \subset \mathrm{N}_{\mathbb{R}}$. More precisely, $V:=\operatorname{Spec} \Lambda\left[\boldsymbol{\sigma}^{\vee} \cap \mathrm{M}\right]$ for the semi-group $\operatorname{ring} \Lambda\left[\boldsymbol{\sigma}^{\vee} \cap \mathrm{M}\right]$, where $\mathrm{M}=\operatorname{Hom}(\mathrm{N}, \mathbb{Z})$ and $\boldsymbol{\sigma}^{\vee}=\left\{m \in \mathrm{M}_{\mathbb{R}} \mid m \geq 0\right.$ on $\left.\boldsymbol{\sigma}\right\}$. Note that $\Lambda\left[\boldsymbol{\sigma}^{\vee} \cap \mathrm{M}_{0}\right]$ is a polynomial algebra $\Lambda\left[\mathrm{x}_{1}, \mathrm{x}_{2}\right]$ of two variables, where $\mathrm{M}_{0}=\operatorname{Hom}\left(\mathrm{N}_{0}, \mathbb{Z}\right)$ and that the subalgebra $\Lambda\left[\boldsymbol{\sigma}^{\vee} \cap \mathrm{M}\right]$ is generated by monomials $\mathrm{x}_{1}^{m_{1}} \mathrm{x}_{2}^{m_{2}}$ for integers $m_{1}$, $m_{2}$ such that $m_{1} \geq 0, m_{2} \geq 0$, and $m_{1}+q m_{2} \equiv 0 \bmod n$. For the group $\operatorname{ring} \Lambda[\mathrm{M}]$, the affine $\Lambda$-scheme $\mathbb{T}_{\mathrm{N}}=\operatorname{Spec} \Lambda[\mathrm{M}]$ is a group scheme isomorphic to $\mathbb{G}_{\mathrm{m}, \Lambda}^{2}$, the algebraic torus of relative dimension two. Since $\boldsymbol{\sigma}^{\vee} \cap \mathrm{M} \subset \mathrm{M}, V$ contains $\mathbb{T}_{\mathrm{N}}(\{0\})=\mathbb{T}_{\mathrm{N}}$ as an open subset. Moreover, the standard action of $\mathbb{T}_{\mathrm{N}}$ on $\mathbb{T}_{\mathrm{N}}$ extends to $V$, compatibly with the open immersion $\mathbb{T}_{\mathrm{N}} \subset V$.

Definition 2.2. The $\Lambda$-scheme $V$ is said to be the toric $\Lambda$-scheme of type $(n, q)$.

For an element $m \in \mathrm{M}$, let $\boldsymbol{e}(m)$ be the element in the group algebra $\Lambda[\mathrm{M}]$ corresponding to $m$. Then, $\boldsymbol{e}(m)$ can be regarded as a rational function on $V$, and it is regular when $m \in \boldsymbol{\sigma}^{\vee} \cap \mathrm{M}$. The cone $\boldsymbol{\sigma}$ has four faces: $\boldsymbol{\sigma}$ itself, two rays $\mathbb{R}_{\geq 0} e_{1}, \mathbb{R}_{\geq 0} e_{2}$, and the zero-dimensional cone $\{0\}$. For such a face $\boldsymbol{\tau}$, let $\boldsymbol{\tau}^{\perp}$ be the vector subspace $\left\{m \in \mathrm{M}_{\mathbb{R}} \mid\right.$ $m(\boldsymbol{\tau})=0\}$ and consider the ring homomorphism $\Lambda\left[\boldsymbol{\sigma}^{\vee} \cap \mathrm{M}\right] \rightarrow \Lambda\left[\boldsymbol{\tau}^{\perp} \cap \mathrm{M}\right]$ defined by

$$
\boldsymbol{e}(m) \mapsto \begin{cases}\boldsymbol{e}(m), & \text { if } m \in \boldsymbol{\tau}^{\perp} \\ 0, & \text { otherwise. }\end{cases}
$$

The kernel of the ring homomorphism defines a closed subscheme $Z(\boldsymbol{\tau})$, which is the closure of an orbit of $\mathbb{T}_{N}$ on $V$. We set $D_{i}:=Z\left(\mathbb{R}_{\geq 0} e_{i}\right)$ for $i=1,2$, and set $\boldsymbol{\Sigma}:=Z(\boldsymbol{\sigma})$. Then, the following properties are easily shown:

- $D_{1}$ and $D_{2}$ are prime divisors on $V$ when $\Lambda$ is integral.

- $\boldsymbol{\Sigma}$ is a section of $V$ over $\operatorname{Spec} \Lambda$.

- $\boldsymbol{\Sigma}$ is the scheme-theoretic intersection $D_{1} \cap D_{2}$ (cf. Remark 2.2.1 below).

- $V \backslash\left(D_{1} \cup D_{2}\right)$ is the "open torus" $\mathbb{T}_{\mathrm{N}}(\{0\})$.

- For $i=1,2, D_{i} \simeq \mathbb{A}_{\Lambda}^{1}$ and $D_{i} \backslash \Sigma \simeq \mathbb{G}_{\mathrm{m}, \Lambda}$.

- $\mathbb{T}_{\mathbf{N}}(\{0\}), D_{1} \backslash \Sigma, D_{2} \backslash \Sigma$, and $\boldsymbol{\Sigma}$ are the orbits of $\mathbb{T}_{\mathrm{N}}$ in $V$.

Note also that, for $i=1,2$, the order of zeros (or the minus of the order of poles) of the rational function $\boldsymbol{e}(m)$ for $m \in \mathrm{M}$ along the prime divisor $D_{i}$ equals $m\left(e_{i}\right)$ (cf. [24, Chapter I, Theorem 9],[11, §5], [41, Section 2.1]). Hence, the principal $\operatorname{divisor} \operatorname{div}(\boldsymbol{e}(m))$ 
has the following expression:

$$
\operatorname{div}(\boldsymbol{e}(m))=m\left(e_{1}\right) D_{1}+m\left(e_{2}\right) D_{2}
$$

Remark 2.2.1. Using ( $(\overline{I I-2})$, we can prove that $\boldsymbol{\Sigma}$ is just the scheme-theoretic intersection of $D_{1}$ and $D_{2}$ as follows. For $i=1,2$, the defining ideal $I_{i}$ of $D_{i}$ is generated by $\boldsymbol{e}(m)$ for $m \in \boldsymbol{\sigma}^{\vee} \cap \mathrm{M}$ with $m\left(e_{i}\right)>0$ by (II-2) . On the other hand, the defining ideal $I$ at $\boldsymbol{\Sigma}$ is generated by $\boldsymbol{e}(m)$ for $m \in \boldsymbol{\sigma}^{\vee} \cap \mathrm{M} \backslash\{0\}$. Thus, $I=I_{1}+I_{2}$, i.e., $\boldsymbol{\Sigma}$ is the scheme-theoretic intersection $D_{1} \cap D_{2}$.

Remark 2.2.2. If $\Lambda^{\prime}$ is a $\Lambda$-algebra, then $V \times_{\operatorname{Spec} \Lambda} \operatorname{Spec} \Lambda^{\prime}$ is also the toric $\Lambda^{\prime}$-scheme of type $(n, q)$. If $\Lambda$ is an algebraically closed field, then $V$ is an affine normal surface with unique singular point $\Sigma$.

In order to show that $\left(V, D_{1}, D_{2}\right)$ satisfies the condition $C(n, q)^{\prime}$, we shall construct the toric "minimal resolution of singularities" $\nu: U \rightarrow V$ which is essentially the same as the well-known Jung-Hirzebruch resolution (cf. [23], [19]). The following lemma follows from the property $n / q=\left[b_{1}, \ldots, b_{l}\right]$ : the proof is left to the reader.

Lemma 2.3. There exist vectors $v_{0}, v_{1}, \ldots, v_{l+1} \in \mathrm{N} \cap \boldsymbol{\sigma}$ satisfying the following conditions:

(1) Let $p_{i}, q_{i}$ be integers determined by $v_{i}=(1 / n)\left(p_{i} e_{1}+q_{i} e_{2}\right)$. Then,

$$
p_{0}=0<p_{1}=1<\cdots<p_{l+1}=n \quad \text { and } \quad q_{0}=n>q_{1}=q>\cdots>q_{l}=1>q_{l+1}=0 .
$$

(2) $v_{i-1}+v_{i+1}=b_{i} v_{i}$ for $1 \leq i \leq l$.

(3) $\mathrm{N}=\mathbb{Z} v_{i-1}+\mathbb{Z} v_{i}$ for $1 \leq i \leq l$.

(4) Let $\left(h_{1}, h_{2}\right)$ be the basis of $\mathrm{M}_{0}=\operatorname{Hom}\left(\mathrm{N}_{0}, \mathbb{Z}\right)$ dual to $\left(e_{1}, e_{2}\right)$, i.e., $h_{i}\left(e_{j}\right)=\delta_{i, j}$. Then, for all $1 \leq i \leq l$,

$$
\left(-q_{i} h_{1}+p_{i} h_{2}, q_{i-1} h_{1}-p_{i-1} h_{2}\right)
$$

is the basis of $\mathrm{M}$ dual to $\left(v_{i-1}, v_{i}\right)$.

(5) $p_{i} q_{j} \equiv p_{j} q_{i} \bmod n$ for all $1 \leq i, j \leq l$.

Note that $v_{0}=e_{2}, v_{1}=v$, and $v_{l+1}=e_{1}$. Note also that the last assertion (5) is derived from (44) since it is equivalent to: $\left(-q_{i} h_{1}+p_{i} h_{2}\right)\left(v_{j}\right) \in \mathbb{Z}$ for all $1 \leq i, j \leq l$.

The cones $\boldsymbol{\sigma}_{i}:=\mathbb{R}_{\geq 0} v_{i-1}+\mathbb{R}_{\geq 0} v_{i}$ for $1 \leq i \leq l$ and the rays $\mathbb{R}_{\geq 0} v_{j}$ for $0 \leq j \leq l+1$ together with the zero cone $\{0\}$ form a non-singular fan $\triangle$ of $N$ such that the support $|\triangle|$ coincides with $\boldsymbol{\sigma}$ (cf. [12, Section 4.2], [24, Chapter I, §2], [11, §5], [41, Chapter 1]). Let $U$ be the toric $\Lambda$-scheme $\mathbb{T}_{N}(\triangle)$ associated with the fan $\triangle$. Then, we have a canonical proper surjective morphism $\nu: U \rightarrow V$ which is an isomorphism at least on the open torus 
$\mathbb{T}_{\mathrm{N}}(\{0\})$. Now, $U$ is a union of the smooth affine toric $\Lambda$-schemes $U_{i}=\operatorname{Spec} \Lambda\left[\boldsymbol{\sigma}_{i}^{\vee} \cap \mathrm{M}\right] \simeq$ $\mathbb{A}_{\Lambda}^{2}$. In fact, by Lemma 2.3 , we see that $\Lambda\left[\boldsymbol{\sigma}_{i}^{\vee} \cap \mathrm{M}\right]$ is a polynomial $\Lambda$-algebra of two variables generated by

$$
\xi_{i}:=\boldsymbol{e}\left(-q_{i} h_{1}+p_{i} h_{2}\right)=\mathrm{x}_{1}^{-q_{i}} \mathrm{x}_{2}^{p_{i}} \quad \text { and } \quad \eta_{i}:=\boldsymbol{e}\left(q_{i-1} h_{1}-p_{i-1} h_{2}\right)=\mathrm{x}_{1}^{q_{i-1}} \mathrm{x}_{2}^{-p_{i-1}} .
$$

Here, $U_{i} \cap U_{j}$ equals the open torus $\mathbb{T}_{\mathrm{N}}(\{0\}) \simeq \mathbb{T}_{\mathrm{N}}$ of $U$ if $|i-j|>1$, since $\boldsymbol{\sigma}_{i} \cap \boldsymbol{\sigma}_{j}=\{0\}$. For $1 \leq i \leq l$, the intersection $U_{i}^{\star}:=U_{i} \cap U_{i+1}$ is isomorphic to the toric $\Lambda$-scheme $\mathbb{T}_{\mathrm{N}}\left(\mathbb{R}_{\geq 0} v_{i}\right) \simeq \mathbb{A}_{\Lambda}^{1} \times_{\text {Spec } \Lambda} \mathbb{G}_{\mathrm{m}, \Lambda}$, since $\boldsymbol{\sigma}_{i} \cap \boldsymbol{\sigma}_{i+1}=\mathbb{R}_{\geq 0} v_{i}$. For $0 \leq i \leq l+1$, let $G_{i}$ be the $\mathbb{T}_{\mathrm{N}}$-invariant prime divisor on $U=\mathbb{T}_{\mathrm{N}}(\triangle)$ corresponding to the ray $\mathbb{R}_{\geq 0} v_{i}$. In particular, $G_{0}$ (resp. $\left.G_{l+1}\right)$ is the proper transform of $D_{2}$ (resp. $D_{1}$ ).

Lemma 2.4. The triplet $\left(V, D_{1}, D_{2}\right)$ satisfies the condition $C(n, q)^{\prime}$ with the proper morphism $\nu: U \rightarrow V$.

Proof. For any $m \in \mathrm{M}$, we have

$$
\operatorname{div}(\boldsymbol{e}(m))=\sum_{i=0}^{l+1} m\left(v_{i}\right) G_{i}
$$

as in (II-2), where $\boldsymbol{e}(m)$ is regarded as a rational function on $U$. Now, $\left.G_{i}\right|_{U_{j}}=0$ for $j<i$ and $j>i+1$, since $v_{i} \notin \boldsymbol{\sigma}_{j}$. Furthermore, we have:

$$
\left.G_{i}\right|_{U_{i}}=\left.\operatorname{div}\left(\eta_{i}\right)\right|_{U_{i}} \quad \text { and }\left.\quad G_{i}\right|_{U_{i+1}}=\left.\operatorname{div}\left(\xi_{i+1}\right)\right|_{U_{i+1}},
$$

by the calculation

$$
\begin{aligned}
& \left.\operatorname{div}\left(\xi_{i}\right)\right|_{U_{i}}=\left.\sum_{j=i-1}^{i}\left(-q_{i} h_{1}+p_{i} h_{2}\right)\left(v_{j}\right) G_{j}\right|_{U_{i}}=\left.G_{i-1}\right|_{U_{i}}, \\
& \left.\operatorname{div}\left(\eta_{i}\right)\right|_{U_{i}}=\left.\sum_{j=i-1}^{i}\left(q_{i-1} h_{1}-p_{i-1} h_{2}\right)\left(v_{j}\right) G_{j}\right|_{U_{i}}=\left.G_{i}\right|_{U_{i}},
\end{aligned}
$$

using (II-3) and Lemma 2.3, As a consequence,

$$
U_{i}^{\star}=U_{i} \backslash G_{i-1}=U_{i} \cap\left\{\xi_{i} \neq 0\right\} \simeq \mathbb{A}_{\Lambda}^{1} \times \mathbb{G}_{\mathrm{m}, \Lambda} .
$$

The toric $\Lambda$-scheme $U$ is obtained by gluing the affine $\Lambda$-planes $U_{i} \simeq \mathbb{A}_{\Lambda}^{2}$ for $1 \leq i \leq l+1$ with the coordinate systems $\left(\xi_{i}, \eta_{i}\right)$. The transition relation is

$$
\left.\xi_{i+1}\right|_{U_{i}^{\star}}=\left.\xi_{i}^{b_{i}} \eta_{i}\right|_{U_{i}^{\star}}, \quad \text { and }\left.\quad \eta_{i+1}\right|_{U_{i}^{\star}}=\left.\xi_{i}^{-1}\right|_{U_{i}^{\star}}
$$

on $U_{i}^{\star}=U_{i} \cap U_{i+1}$. This is possible, since $\left(-q_{i+1}, p_{i+1}\right)-\left(q_{i-1},-p_{i-1}\right)=b_{i}\left(-q_{i}, p_{i}\right)$ (cf. Lemma 2.3). Furthermore, $G_{i} \simeq \mathbb{P}_{\Lambda}^{1}$ with $\mathcal{O}_{G_{i}}\left(G_{i}\right) \simeq \mathcal{O}\left(-b_{i}\right)$ for all $1 \leq i \leq l$ by (II-4) and (II-5) . On the other hand, $G_{0}$ and $G_{l+1}$ are isomorphic to $\mathbb{A}_{\Lambda}^{1}$. The scheme-theoretic intersection $\Sigma_{i}=G_{i-1} \cap G_{i}$ for $1 \leq i \leq l+1$ is just the orbit corresponding to the twodimensional cone $\boldsymbol{\sigma}_{i}$. Therefore, $\sum_{i=0}^{l+1} G_{i}$ is a (relative) simple normal crossing divisor (over Spec $\Lambda$ ) with the dual graph similar to (II-1), and hence, $\left(V, D_{1}, D_{2}\right)$ satisfies the condition $C(n, q)^{\prime}$. 
Remark 2.4.1. Let $p_{i}$ and $q_{i}$ for $0 \leq i \leq l+1$ be the integers defined by $(n, q)$ in Lemma 2.3 . If $a_{1}$ and $a_{2}$ are integers with $a_{1}+q a_{2} \equiv 0 \bmod n$, then, $p_{i} a_{1}+q_{i} a_{2} \equiv 0 \bmod n$ for all $0 \leq i \leq l+1$, and

$$
\mu^{*}\left(a_{1} D_{1}+a_{2} D_{2}\right)=\sum_{i=0}^{l+1} \frac{p_{i} a_{1}+q_{i} a_{2}}{n} G_{i}
$$

In fact, this is derived from (ㅍ-2) and (II-3) applied to $m=a_{1} h_{1}+a_{2} h_{2}$. As special cases, we have

$$
\mu^{*}\left(n D_{1}\right)=\sum_{i=1}^{l+1} p_{i} G_{i} \quad \text { and } \quad \mu^{*}\left(n D_{2}\right)=\sum_{i=0}^{l} q_{i} G_{i}
$$

We have another proof of $(\underline{\underline{I I}-6})$ in Corollary 2.8 .3 below.

Next, we consider an arbitrary affine $\Lambda$-scheme $Y$ and a closed subscheme $\Sigma$ satisfying $C(n, q)$. First we shall show:

Lemma 2.5. Let $Y$ be an affine $\Lambda$-scheme and $\Sigma$ a closed subscheme such that $(Y, \Sigma)$ satisfies $C(n, q)$ for a proper morphism $\mu: M \rightarrow Y$. Then, $\mathcal{O}_{Y} \simeq \mu_{*} \mathcal{O}_{M} \simeq j_{*} \mathcal{O}_{Y \backslash \Sigma}$, where $j: Y \backslash \Sigma \hookrightarrow Y$ denotes the open immersion.

Proof. We take an arbitrary point $y \in \Sigma$ and a point $x \in M$ lying over $y$. Let $t \in \operatorname{Spec} \Lambda$ be the image of $y$, and let $Y_{t}$ and $M_{t}$ be the fiber over $t$ of $Y \rightarrow \operatorname{Spec} \Lambda$ and $M \rightarrow \operatorname{Spec} \Lambda$, respectively. Then,

$$
\begin{aligned}
\operatorname{depth} \mathcal{O}_{Y, y} & =\operatorname{depth} \mathcal{O}_{Y_{t}, y}+\operatorname{depth} \mathcal{O}_{\text {Spec } \Lambda, t} \geq \operatorname{depth} \mathcal{O}_{Y_{t}, y}=2, \\
\operatorname{depth} \mathcal{O}_{M, x} & =\operatorname{depth} \mathcal{O}_{M_{t}, x}+\operatorname{depth} \mathcal{O}_{\text {Spec } \Lambda, t} \geq \operatorname{depth} \mathcal{O}_{M_{t}, x} \geq 1
\end{aligned}
$$

since $Y$ and $M$ are flat over $\Lambda$, and since all the fibers of $Y \rightarrow \operatorname{Spec} \Lambda$ and $M \rightarrow \operatorname{Spec} \Lambda$ are normal surfaces. Hence, $\mathcal{O}_{Y} \rightarrow j_{*} \mathcal{O}_{Y \backslash \Sigma}$ is an isomorphism and $\mathcal{O}_{M} \rightarrow j_{*}^{\prime} \mathcal{O}_{M \backslash \mu^{-1}(\Sigma)}$ is injective for the open immersion $j^{\prime}: M \backslash \mu^{-1}(\Sigma) \hookrightarrow M$ (cf. [EGA, IV, Théorème (5.10.5), Proposition (5.10.2)] or [SGA2, Exp. III, Proposition 3.3, Corollaire 3.5]). Since $M \backslash$ $\mu^{-1}(\Sigma) \simeq Y \backslash \Sigma$, we have isomorphisms $\mathcal{O}_{Y} \simeq \mu_{*} \mathcal{O}_{M} \simeq j_{*} \mathcal{O}_{Y \backslash \Sigma}$

The condition $C(n, q)$ characterizes the toric $\Lambda$-scheme of type $(n, q)$ up to étale morphism when $\Lambda$ is a field. Namely, we have:

Theorem 2.6 (tautness). Assume that $\Lambda$ is a field. Let $(Y, \Sigma)$ be a pair satisfying the condition $C(n, q)$ with a proper morphism $\mu: M \rightarrow Y$. Let $V$ be the toric $\Lambda$-scheme $V$ of type $(n, q)$ and $\nu: U \rightarrow V$ the minimal resolution constructed as above. Then, there exists an étale neighborhood $Y^{\circ}$ of $\Sigma$ in $Y$ with étale morphisms $\tau: Y^{\circ} \rightarrow V$ and 
$\Phi: M^{\circ}:=M \times_{Y} Y^{\circ} \rightarrow U$ which form a commutative Cartesian diagram:

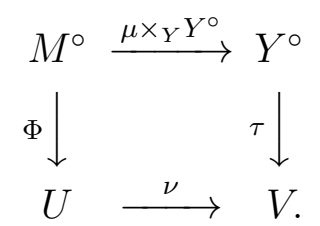

Remark 2.6.1. An étale neighborhood $Y^{\circ}$ of $\Sigma$ in $Y$ means an étale morphism $Y^{\circ} \rightarrow Y$ such that the image contains $\Sigma$ and that $\Sigma \times_{Y} Y^{\circ} \rightarrow \Sigma$ is an isomorphism (cf. Notation and conventions).

Similarly, the condition $C(n, q)^{\prime}$ characterizes the toric $\Lambda$-scheme of type $(n, q)$ up to étale morphism:

Theorem 2.7. Assume that $\Lambda$ is a Noetherian local ring. Let $\left(Y, B_{1}, B_{2}\right)$ be a collection satisfying the condition $C(n, q)^{\prime}$ with a proper morphism $\mu: M \rightarrow Y$. Let $V$ be the toric $\Lambda$-scheme $V$ of type $(n, q)$ and $\nu: U \rightarrow V$ the minimal resolution constructed as above. Then, there exists an open neighborhood $Y^{\circ}$ of $\Sigma$ in $Y$ with étale morphisms $\tau: Y^{\circ} \rightarrow V$ and $\Phi: M^{\circ}:=M \times_{Y} Y^{\circ} \rightarrow U$ which form the same commutative Cartesian diagram as in Theorem 2.6.

Before proving Theorems 2.6 and 2.7, we need some results on the "singularity" of $Y$ along $\Sigma$.

Lemma 2.8. In the situation of Theorem 2.6 or 2.7, if an invertible sheaf $\mathcal{L}$ on $M$ is $\mu$-nef, i.e., $\operatorname{deg}\left(\left.\mathcal{L}\right|_{C}\right) \geq 0$ for any fiber $C$ of $E_{i} \simeq \mathbb{P}_{\Lambda}^{1} \rightarrow \Sigma \simeq \operatorname{Spec} \Lambda$ for all $1 \leq i \leq l$, then $\mathrm{H}^{j}(M, \mathcal{L})=0$ for all $j>0$ and $\mathcal{L}$ is generated by global sections. If $\mathcal{L}$ is $\mu$-numerically trivial, i.e., $\operatorname{deg}\left(\left.\mathcal{L}\right|_{C}\right)=0$ for any $C$ above, then $\mu_{*} \mathcal{L}$ is an invertible sheaf on $Y$ and $\mu^{*}\left(\mu_{*} \mathcal{L}\right) \simeq \mathcal{L}$

Proof. For the vanishing $\mathrm{H}^{j}(M, \mathcal{L})=0$, it is enough to check only when $j=1$, since the dimension of the fibers of $\mu$ is at most one. Let $Z$ be the divisor $\sum_{i=1}^{l} E_{i}$ which is a relative Cartier divisor over $\operatorname{Spec} \Lambda$. Then, $\mathcal{O}_{M}(-Z)$ is $\mu$-nef by $b_{i} \geq 2$. It is enough to prove the following two assertions:

(1) $\mathrm{H}^{1}\left(Z,\left.\mathcal{L}\right|_{Z}\right)=0$.

(2) $\left.\mathcal{L}\right|_{Z}$ is generated by global sections.

In fact, $\mathrm{H}^{1}\left(Z,\left.\mathcal{L}\right|_{Z} \otimes \mathcal{O}_{Z}(-m Z)\right)=0$ for any $m \geq 0$ by (11), and it implies the vanishing $\mathrm{H}^{1}\left(M, \mathcal{O}_{m Z} \otimes \mathcal{L}\right)=0$ for all $m \geq 0$ by induction using the exact sequence

$$
0 \rightarrow \mathcal{O}_{Z}(-(m-1) Z) \otimes_{\mathcal{O}_{M}} \mathcal{L} \rightarrow \mathcal{O}_{m Z} \otimes_{\mathcal{O}_{M}} \mathcal{L} \rightarrow \mathcal{O}_{(m-1) Z} \otimes_{\mathcal{O}_{M}} \mathcal{L} \rightarrow 0 .
$$

Hence, $\mathrm{H}^{1}(M, \mathcal{L})=0$, since the module $\mathrm{H}^{1}(M, \mathcal{L})$ is supported on $\Sigma$ and the formal completion $\mathrm{H}^{1}(M, \mathcal{L})^{\wedge}$ along $\Sigma$ is isomorphic to the projective limit $\varliminf_{m} \mathrm{H}^{1}\left(\mathcal{O}_{m Z} \otimes \mathcal{L}\right)$ 
(cf. [EGA, III, Théorème (4.1.5)]). We also have $\mathrm{H}^{1}\left(M, \mathcal{O}_{M}(-Z) \otimes \mathcal{L}\right)=0$, since $\mathcal{O}_{M}(-Z)$ is nef, and as a consequence, the restriction map $\mathrm{H}^{0}(M, \mathcal{L}) \rightarrow \mathrm{H}^{0}\left(Z,\left.\mathcal{L}\right|_{Z}\right)$ is surjective. Thus, $\mathcal{L}$ is generated by global sections by (21).

For integers $1 \leq a \leq b \leq l$, we set $Z_{a, b}:=\sum_{i=a}^{b} E_{i}$. We shall show (11) and (2) by proving the following two assertions for any such pair $(a, b)$ of integers:

(3) $\mathrm{H}^{1}\left(Z_{a, b},\left.\mathcal{L}\right|_{Z_{a, b}}\right)=0$.

(4) $\left.\mathcal{L}\right|_{Z_{a, b}}$ is generated by global sections.

We shall prove them by induction on $b-a$. These are true when $a=b$. In fact, $Z_{a, a}=E_{a}$, $E_{a} \simeq \mathbb{P}_{\Lambda}^{1}$, and $\left.\mathcal{L}\right|_{E_{a}} \simeq \mathcal{O}_{\mathbb{P}_{\Lambda}^{1}}\left(d_{a}\right)$ for some $d_{a} \geq 0$. Assume that $b>a$. Then, we have two exact sequences

$$
\begin{aligned}
& 0 \rightarrow \mathcal{O}_{E_{a}}\left(-Z_{a+1, b}\right) \simeq \mathcal{O}_{\mathbb{P}_{\Lambda}^{1}}(-1) \rightarrow \mathcal{O}_{Z_{a, b}} \rightarrow \mathcal{O}_{Z_{a+1, b}} \rightarrow 0 \text { and } \\
& 0 \rightarrow \mathcal{O}_{E_{b}}\left(-Z_{a, b-1}\right) \simeq \mathcal{O}_{\mathbb{P}_{\Lambda}^{1}}(-1) \rightarrow \mathcal{O}_{Z_{a, b}} \rightarrow \mathcal{O}_{Z_{a, b-1}} \rightarrow 0 .
\end{aligned}
$$

Since $\left.\mathcal{L}\right|_{E_{a}} \simeq \mathcal{O}_{\mathbb{P}_{\Lambda}^{1}}\left(d_{a}\right)$ and $\left.\mathcal{L}\right|_{E_{b}} \simeq \mathcal{O}_{\mathbb{P}_{\Lambda}^{1}}\left(d_{b}\right)$ with $d_{a}, d_{b} \geq 0$, we have

$$
\mathrm{H}^{1}\left(E_{a},\left.\mathcal{L}\right|_{E_{a}} \otimes \mathcal{O}_{E_{a}}\left(-Z_{a+1, b}\right)\right)=\mathrm{H}^{1}\left(E_{b},\left.\mathcal{L}\right|_{E_{b}} \otimes \mathcal{O}_{E_{b}}\left(-Z_{a, b-1}\right)\right)=0
$$

Therefore, we have surjections $\mathrm{H}^{0}\left(\left.\mathcal{L}\right|_{Z_{a, b}}\right) \rightarrow \mathrm{H}^{0}\left(\left.\mathcal{L}\right|_{Z_{a+1, b}}\right)$ and $\mathrm{H}^{0}\left(\left.\mathcal{L}\right|_{Z_{a, b}}\right) \rightarrow \mathrm{H}^{0}\left(\left.\mathcal{L}\right|_{Z_{a, b-1}}\right)$, and isomorphisms $\mathrm{H}^{1}\left(\left.\mathcal{L}\right|_{Z_{a, b}}\right) \simeq \mathrm{H}^{1}\left(\left.\mathcal{L}\right|_{Z_{a+1, b}}\right) \simeq \mathrm{H}^{1}\left(\left.\mathcal{L}\right|_{Z_{a, b-1}}\right)$. Thus, (3) and (41) for $(a, b)$ follow from those for $(a+1, b)$ and for $(a, b-1)$. By induction on $b-a$, (3) and (44) hold for any $(a, b)$, and hence, (11) and (2) hold. Thus, we are done.

Remark. If one knows the vanishing $\mathrm{H}^{1}\left(M, \mathcal{O}_{M}\right)=0$, then Lemma 2.8 is a special case of [36, Theorem (12.1)], which generalizes [1, Lemma 5] stated over an algebraically closed field.

Corollary 2.8.1. Let $Y$ be an affine normal algebraic surface defined over an algebraically closed field $\mathbb{k}$ with a unique singular point $P$. If the exceptional locus for the minimal resolution of a singularity is a linear chain of smooth rational curves, then $(Y, P)$ is a rational singularity.

Remark 2.8.2. In Corollary 2.8.1, the divisor $Z$ in the proof of Lemma 2.8 is nothing but the fundamental cycle. Thus, the rationality of $(Y, P)$ is also derived from $p_{a}(Z)=0$ by [1, Theorem 3].

Corollary 2.8.3. Let $\mu: M \rightarrow Y, B_{1}, B_{2}$, and $\left\{E_{i}\right\}_{i=0}^{l+1}$ be as in the situation of Theorem 2.7. Let $a_{1}, a_{2}$ be integers such that $a_{1}+q a_{2} \equiv 0 \bmod n$. Then, $p_{i} a_{1}+q_{i} a_{2} \equiv 0 \bmod n$ for all $0 \leq i \leq l+1$ for integers $p_{i}$ and $q_{i}$ defined in Lemma 2.3 , $a_{1} B_{1}+a_{2} B_{2}$ is Cartier, and the equality

$$
\mu^{*}\left(a_{1} B_{1}+a_{2} B_{2}\right)=\sum_{i=0}^{l+1} \frac{p_{i} a_{1}+q_{i} a_{2}}{n} E_{i}
$$


of Cartier divisors hold on $M$.

Proof. By Lemma 2.3, we have $p_{i} a_{1}+q_{i} a_{2} \equiv 0 \bmod n$, and we see that the right hand side of (II-8) is $\mu$-numerically trivial. Hence by Lemma 2.8 , the associated invertible sheaf to the divisor of the right hand side is just the pullback of an invertible sheaf on $Y$. Thus, (II-8) holds, since $a_{1} B_{1}+a_{2} B_{2}$ is the push-forward of the right hand side.

Proof of Theorems 2.6 and 2.7. First, we shall prove Theorem 2.6 assuming Theorem 2.7 to be true. In Theorem 2.6, $\Lambda$ is a field, say $\mathbb{K}$. Thus, $\Sigma$ is a $\mathbb{K}$-rational point. Let $R$ be the henselization of the local ring $\mathcal{O}_{Y, \Sigma}$ and let $\mu^{\sim}: M^{\sim} \rightarrow$ Spec $R$ be the base change of $\mu: M \rightarrow Y$ by $\operatorname{Spec} R \rightarrow Y$. Then,

$$
\operatorname{Pic}\left(M^{\sim}\right) \rightarrow \operatorname{Pic}\left(\bigcup_{i=1}^{l} E_{i}\right)
$$

is surjective by [36, Lemma (14.3)] or [EGA, IV, Corollaire (21.9.12)]. Hence, by replacing $Y$ with an étale neighborhood $Y^{\circ} \rightarrow Y$ of $\Sigma$ in $Y$, we may assume that $\operatorname{Pic}(M) \rightarrow$ $\operatorname{Pic}\left(\bigcup_{i=1}^{l} E_{i}\right)$ is surjective. Then, we can find invertible sheaves $\mathcal{L}, \mathcal{L}^{\prime}$ on $M$ such that

$$
\left.\operatorname{deg} \mathcal{L}\right|_{E_{1}}=\left.\operatorname{deg} \mathcal{L}^{\prime}\right|_{E_{l}}=1, \quad \text { and }\left.\quad \operatorname{deg} \mathcal{L}\right|_{E_{i}}=\left.\operatorname{deg} \mathcal{L}^{\prime}\right|_{E_{j}}=0
$$

for $i>1$ and $j<l$. Applying Lemma 2.8 to $\mathcal{L}$ and $\mathcal{L}^{\prime}$, we have two affine prime divisors $E_{0}, E_{l+1}$ on $M$ such that

- $\mathcal{L} \simeq \mathcal{O}_{M}\left(E_{0}\right)$ and $\mathcal{L}^{\prime} \simeq \mathcal{O}_{M}\left(E_{l+1}\right)$,

- $E_{0} \cap E_{1}$ and $E_{l} \cap E_{l+1}$ are sections of $M \rightarrow \operatorname{Spec} \Lambda$, and

- $E_{0} \cap \bigcup_{i=2}^{l+1} E_{i}=E_{l+1} \cap \bigcup_{i=0}^{l-1} E_{i}=\emptyset$.

Hence, the dual graph of $\sum_{i=0}^{l+1} E_{i}$ is the same as (II-1). We set $B_{1}:=\mu_{*}\left(E_{l+1}\right)$ and $B_{2}:=\mu_{*}\left(E_{0}\right)$. Then, $B_{1}$ and $B_{2}$ are prime divisors on $Y$ with $B_{1} \cap B_{2}=\Sigma$, settheoretically. Furthermore, $E_{l+1}$ and $E_{0}$ are the proper transforms of $B_{1}$ and $B_{2}$, respectively. Thus, $\left(Y, B_{1}, B_{2}\right)$ satisfies the condition $C(n, q)^{\prime}$. Hence, Theorem [2.6] is derived from Theorem 2.7 .

In the rest of the proof, we shall prove Theorem 2.7. If $a_{1}$ and $a_{2}$ are integers with $a_{1}+q a_{2} \equiv 0 \bmod n$, then $a_{1} B_{1}+a_{2} B_{2}$ is Cartier by Corollary 2.8.3. We may assume that these Cartier divisors $a_{1} B_{1}+a_{2} B_{2}$ are all linearly equivalent to zero by replacing $Y$ with an open neighborhood of $\Sigma$, since $\Lambda$ is local.

For $0 \leq i \leq l+1$, let $\epsilon_{i}$ be a global section of $\mathcal{O}_{M}\left(E_{i}\right)$ such that the divisor $\left(\epsilon_{i}\right)_{0}$ of zeros equals $E_{i}$; in other words, $\epsilon_{i}: \mathcal{O}_{M} \rightarrow \mathcal{O}_{M}\left(E_{i}\right)$ is dual to the natural injection $\mathcal{O}_{M}\left(-E_{i}\right) \subset \mathcal{O}_{M}$. Let $a_{1}, a_{2}$ be integers with $a_{1}+q a_{2} \equiv 0 \bmod n$. Then, by (II-8), there is a rational function $\phi_{a_{1}, a_{2}}$ on $Y$ such that

$$
\mu^{*}\left(\phi_{a_{1}, a_{2}}\right)=\prod_{i=0}^{l+1} \epsilon_{i}^{\left(p_{i} a_{1}+q_{i} a_{2}\right) / n} .
$$


For $1 \leq i \leq l+1$, let $M_{i}$ be the complement of $\bigcup_{k \neq i-1, i} E_{k}$ in $M$. Then, $M_{i}$ is a neighborhood of the intersection $E_{i-1} \cap E_{i}$. We define

$$
s_{i}:=\left.\mu^{*}\left(\phi_{-q_{i}, p_{i}}\right)\right|_{M_{i}} \quad \text { and } \quad t_{i}:=\left.\mu^{*}\left(\phi_{q_{i-1},-p_{i-1}}\right)\right|_{M_{i}}
$$

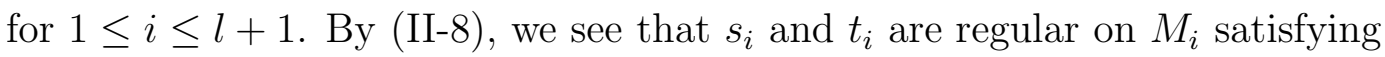

$$
\left.\operatorname{div}\left(s_{i}\right)\right|_{M_{i}}=\left.E_{i-1}\right|_{M_{i}} \quad \text { and }\left.\quad \operatorname{div}\left(t_{i}\right)\right|_{M_{i}}=\left.E_{i}\right|_{M_{i}} .
$$

In particular, $\left(s_{i}, t_{i}\right)$ is a local coordinate system of $M_{i}$ along the intersection $E_{i-1} \cap E_{i}$. For $1 \leq i \leq l$, let $M_{i}^{\star}$ be the intersection $M_{i} \cap M_{i+1}=M \backslash \bigcup_{k \neq i} E_{k}$. Then, we have

$$
\left.s_{i+1}\right|_{M_{i}^{\star}}=\left.s_{i}^{b_{i}} t_{i}\right|_{M_{i}^{\star}},\left.\quad t_{i+1}\right|_{M_{i}^{\star}}=\left.s_{i}^{-1}\right|_{M_{i}^{\star}},
$$

similar to ([I-5). For $1 \leq i \leq l+1$, let $\Phi_{i}: M_{i} \rightarrow U_{i}=\operatorname{Spec} \mathbb{k}\left[\boldsymbol{\sigma}_{i}^{\vee} \cap \mathrm{M}\right] \simeq \mathbb{A}_{\mathrm{k}}^{2}$ be the morphism defined by

$$
\Phi_{i}^{*}\left(\xi_{i}\right)=s_{i} \quad \text { and } \quad \Phi_{i}^{*}\left(\eta_{i}\right)=t_{i},
$$

which is étale along $\left(E_{i-1} \cup E_{i}\right) \cap M_{i}$. By (II-5) and (II-9), the morphisms $\Phi_{i}$ for $1 \leq i \leq$ $l+1$ are glued to a morphism $\Phi: M=\bigcup M_{i} \rightarrow U=\bigcup U_{i}$, which is étale along $\bigcup_{i=0}^{l+1} E_{i}$, and which induces $\Phi^{*}\left(G_{i}\right)=E_{i}$ for all $0 \leq i \leq l+1$. Since $\mu \circ \Phi: M \rightarrow U \rightarrow V$ contracts the divisor $E=\sum_{i=1}^{l} E_{i}$ to the section $\Sigma$, we have a morphism $\tau: Y \rightarrow V$ such that $\nu \circ \Phi=\tau \circ \mu$, i.e., the diagram

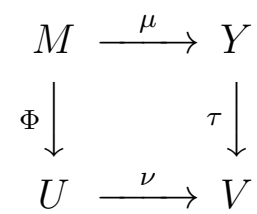

is commutative. For the proof of Theorem 2.7, it is enough to prove that $\tau$ is étale along $\Sigma$. Applying [EGA, III, Théorème (4.1.5)], we have isomorphisms

$$
\mathcal{O}_{Y}^{\wedge} \simeq \lim _{m} \mu_{*}\left(\mathcal{O}_{M} / \mathcal{O}_{M}(-m E)\right) \quad \text { and } \quad \mathcal{O}_{V}^{\wedge} \simeq \lim _{m} \nu_{*}\left(\mathcal{O}_{U} / \mathcal{O}_{U}(-m G)\right)
$$

where $E=\sum_{i=1}^{l} E_{i}, G=\sum_{i=1}^{l} G_{i}$, and $\mathcal{O}_{Y}^{\wedge}$ (resp. $\mathcal{O}_{V}^{\wedge}$ ) is the formal completion of $\mathcal{O}_{Y} \simeq \mu_{*} \mathcal{O}_{M}\left(\operatorname{resp} . \mathcal{O}_{V} \simeq \nu_{*} \mathcal{O}_{U}\right)$ along $\Sigma(\operatorname{resp} . \boldsymbol{\Sigma})$. On the other hand, $\Phi$ induces an isomorphism

$$
\mathrm{H}^{0}\left(U, \mathcal{O}_{U} / \mathcal{O}_{U}(-m G)\right) \simeq \mathrm{H}^{0}\left(M, \mathcal{O}_{M} / \mathcal{O}_{M}(-m E)\right)
$$

of $\Lambda$-algebras for all $m>0$, since $\Phi^{*}(G)=E$ and $\left.\Phi\right|_{E}: E \rightarrow G$ is an isomorphism. Thus, $\tau$ induces an isomorphism $\mathcal{O}_{U}^{\wedge} \simeq \mathcal{O}_{Y}^{\wedge}$. Hence, the morphism $\tau_{t}: Y_{t} \rightarrow V_{t}$ between the fibers of $V$ and $U$ over any point $t \in \operatorname{Spec} \Lambda$ induced by $\tau$ is étale at the point $\Sigma \cap Y_{t}$ by [EGA, IV, Théorème (17.6.1) or Proposition (17.6.3)]. Thus, $\tau$ is étale along $\Sigma$ by [EGA, IV, Proposition (17.8.2)], and this completes the proof. 
In the rest of Section 2, we shall show some local properties of surface singularities that have a linear chain of smooth rational curves as the exceptional divisor for the minimal resolution.

Lemma 2.9. Let $V$ be the toric surface $\mathbb{T}_{\mathrm{N}}(\boldsymbol{\sigma})$ of type $(n, q)$ defined over $\mathbb{k}$ and let $D_{1}$, $D_{2}, \nu: U \rightarrow V, G_{i}$ be the same as above, except that the singular point of $V$ is denoted by $\mathbf{0}$ instead of $\boldsymbol{\Sigma}$. Then, there are natural isomorphisms:

$$
\begin{aligned}
\nu_{*} \mathcal{O}_{U}\left(G_{0}\right) & \simeq \mathcal{O}_{V}\left(D_{2}\right), & \nu_{*} \mathcal{O}_{G_{0}}\left(G_{0}\right) & \simeq \mathcal{E} x t_{\mathcal{O}_{V}}^{1}\left(\mathcal{O}_{D_{2}}, \mathcal{O}_{V}\right) \\
\nu_{*} \mathcal{O}_{U}\left(G_{l+1}\right) & \simeq \mathcal{O}_{V}\left(D_{1}\right), & \nu_{*} \mathcal{O}_{G_{l+1}}\left(G_{l+1}\right) & \simeq \mathcal{E} x t_{\mathcal{O}_{V}}^{1}\left(\mathcal{O}_{D_{1}}, \mathcal{O}_{V}\right)
\end{aligned}
$$

Proof. Let $\mathbb{k}(V)=\mathbb{k}(U)$ be the rational function field of $V$ and $U$. For a non-zero rational function $\varphi \in \mathbb{k}(V)$, if $\operatorname{div}(\varphi)_{V}+D_{2} \geq 0$ for the principal $\operatorname{divisor} \operatorname{div}(\varphi)_{V}$ on $V$, then we have

$$
n \operatorname{div}(\varphi)_{U}+n G_{0}=\nu^{*}\left(\operatorname{div}\left(\varphi^{n}\right)_{V}+n D_{2}\right)-\sum_{i=1}^{l} q_{i} G_{i} \geq-\sum_{i=1}^{l} q_{i} G_{i}
$$

for the principal divisor $\operatorname{div}(\varphi)_{U}$ on $U$ by applying (II-7) in Remark 2.4.1. Thus, $\operatorname{div}(\varphi)_{U}+$ $G_{0} \geq 0$, since $q_{i}<n$ for all $1 \leq i \leq l$. On the other hand, if a non-zero rational function $\psi \in \mathbb{k}(U)$ satisfies $\operatorname{div}(\psi)_{U}+G_{0} \geq 0$, then

$$
\operatorname{div}(\psi)_{V}+D_{2}=\nu_{*}\left(\operatorname{div}(\psi)_{U}+G_{0}\right) \geq 0 .
$$

Therefore, $\mathrm{H}^{0}\left(V, \mathcal{O}_{V}\left(D_{2}\right)\right)=\mathrm{H}^{0}\left(U, \mathcal{O}_{U}\left(G_{0}\right)\right)$, and we have an isomorphism $\nu_{*} \mathcal{O}_{U}\left(G_{0}\right) \simeq$ $\mathcal{O}_{V}\left(D_{2}\right)$. By applying $\nu_{*}$ to the exact sequence $0 \rightarrow \mathcal{O}_{U} \rightarrow \mathcal{O}_{U}\left(G_{0}\right) \rightarrow \mathcal{O}_{G_{0}}\left(G_{0}\right) \rightarrow 0$, we have another exact sequence

$$
0 \rightarrow \mathcal{O}_{V} \rightarrow \mathcal{O}_{V}\left(D_{2}\right) \rightarrow \nu_{*} \mathcal{O}_{G_{0}}\left(G_{0}\right) \rightarrow 0
$$

since $\mathrm{R}^{1} \nu_{*} \mathcal{O}_{U}=0$ by the rationality of the toric singularity or by Corollary 2.8.1. Hence, $\nu_{*} \mathcal{O}_{G_{0}}\left(G_{0}\right) \simeq \mathcal{E} x t_{\mathcal{O}_{V}}^{1}\left(\mathcal{O}_{D_{2}}, \mathcal{O}_{V}\right)$ is derived from $0 \rightarrow \mathcal{O}_{V}\left(-D_{2}\right) \rightarrow \mathcal{O}_{V} \rightarrow \mathcal{O}_{D_{2}} \rightarrow 0$ by applying $\mathcal{E} x t_{\mathcal{O}_{V}}^{i}\left(\bullet, \mathcal{O}_{V}\right)$. The other isomorphisms concerning $G_{l+1}$ and $D_{1}$ are obtained by a similar way.

Before going to Lemma 2.10, we recall some basics on the "sheaf of logarithmic oneforms" on the toric surface $V=\mathbb{T}_{\mathrm{N}}(\boldsymbol{\sigma})$ and the residue homomorphism. The sheaf $\Omega_{\mathbb{T}_{\mathrm{N}} / \mathbb{k}}^{1}$ of one-forms on the torus $\mathbb{T}_{N}=\operatorname{Spec} \mathbb{k}[\mathrm{M}]$ is trivial by the isomorphism

$$
\mathrm{M} \otimes_{\mathbb{Z}} \mathcal{O}_{\mathbb{T}_{\mathrm{N}}} \stackrel{\simeq}{\rightarrow} \Omega_{\mathbb{T}_{\mathrm{N}} / \mathrm{k}}^{1}
$$

which maps $m \otimes 1$ to $\boldsymbol{e}(m)^{-1} \mathrm{~d} \boldsymbol{e}(m)$ for any $m \in \mathrm{M}$. Regarding $\mathbb{T}_{\mathrm{N}}$ as the open subset $\mathbb{T}_{\mathrm{N}}(\{0\})=V \backslash D$ of $V=\mathbb{T}_{\mathrm{N}}(\boldsymbol{\sigma})$, where $D=D_{1}+D_{2}$, we can extend the isomorphism to

$$
\theta: \mathrm{M} \otimes_{\mathbb{Z}} \mathcal{O}_{V} \stackrel{\simeq}{\rightarrow} \widetilde{\Omega}_{V / \mathbb{k}}^{1}(\log D):=j_{*} \Omega_{V^{\circ} / \mathbb{k}}^{1}\left(\log D^{\circ}\right),
$$


where $j$ is the open immersion $V^{\circ}:=V \backslash\{\mathbf{0}\} \subset V$, and $D^{\circ}$ is the divisor $\left.D\right|_{V^{\circ}}$ (cf. [21, (1.12), Proposition], [15, $\S 4.3$, Proposition]). Note that $V^{\circ}$ and $D^{\circ}$ are non-singular. The logarithmic tangent sheaf

$$
\Theta_{V / \mathbb{k}}(-\log D):=\mathcal{H o m}_{\mathcal{O}_{V}}\left(\widetilde{\Omega}_{V / \mathbb{k}}^{1}(\log D), \mathcal{O}_{V}\right)
$$

is isomorphic to $\mathbf{N} \otimes_{\mathbb{Z}} \mathcal{O}_{V}$ by the dual of $\theta$. The double-dual $\left(\Omega_{V / \mathbb{k}}^{1}\right)^{\vee \vee}$ of the sheaf $\Omega_{V / \mathbb{k}}^{1}$ of relative one-forms is just the kernel of the residue homomorphism

$$
\operatorname{Res}: \widetilde{\Omega}_{V / \mathbb{k}}^{1}(\log D) \rightarrow \mathcal{O}_{D_{1}} \oplus \mathcal{O}_{D_{2}}
$$

This residue homomorphism is given by the evaluation map

$$
\text { ev : } \mathrm{M} \ni m \mapsto\left(m\left(e_{1}\right), m\left(e_{2}\right)\right) \in \mathbb{Z} \oplus \mathbb{Z}
$$

in the sense that there is a commutative diagram

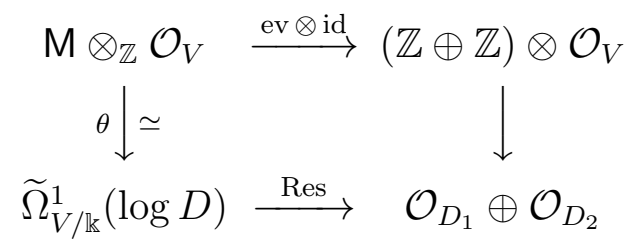

where the right vertical arrow is the direct sum of the natural homomorphisms $\mathcal{O}_{V} \rightarrow \mathcal{O}_{D_{1}}$ and $\mathcal{O}_{V} \rightarrow \mathcal{O}_{D_{2}}$.

Lemma 2.10. The residue homomorphism Res is surjective if and only if $p \nmid n$, where $p=$ char $\mathbb{k}$. If $p \mid n$, then the cokernel of Res is the skyscraper sheaf at $\mathbf{0}$ corresponding to the residue field $\mathbb{k}(\mathbf{0})$ of $\mathcal{O}_{V, \mathbf{0}}$, and the image of Res is isomorphic to $\mathcal{O}_{D}$.

Proof. The subgroup $\mathrm{M}$ of $\mathrm{M}_{0}=\mathbb{Z} h_{1}+\mathbb{Z} h_{2}$ is generated by elements $a_{1} h_{1}+a_{2} h_{2}$ such that $a_{1}+q a_{2} \equiv 0 \bmod n$. Here, Res $\circ \theta$ maps $\left(a_{1} h_{1}+a_{2} h_{2}\right) \otimes 1$ to $\left(a_{1}, a_{2}\right) \in \mathrm{H}^{0}\left(D_{1}, \mathcal{O}_{D_{1}}\right) \oplus$ $\mathrm{H}^{0}\left(D_{2}, \mathcal{O}_{D_{2}}\right)$. We shall show that the cokernel of Res is isomorphic to $\mathbb{k}(\mathbf{0}) / n \mathbb{k}(\mathbf{0})$ by the homomorphism

$$
\phi: \mathcal{O}_{D_{1}} \oplus \mathcal{O}_{D_{2}} \ni\left(\alpha_{1}, \alpha_{2}\right) \mapsto\left(\left.\alpha_{1}\right|_{\mathbf{0}}+\left.q \alpha_{2}\right|_{\mathbf{0}}\right) \bmod n \in \mathbb{k}(\mathbf{0}) / n \mathbb{k}(\mathbf{0}) .
$$

The homomorphism $\phi$ is in the commutative diagram

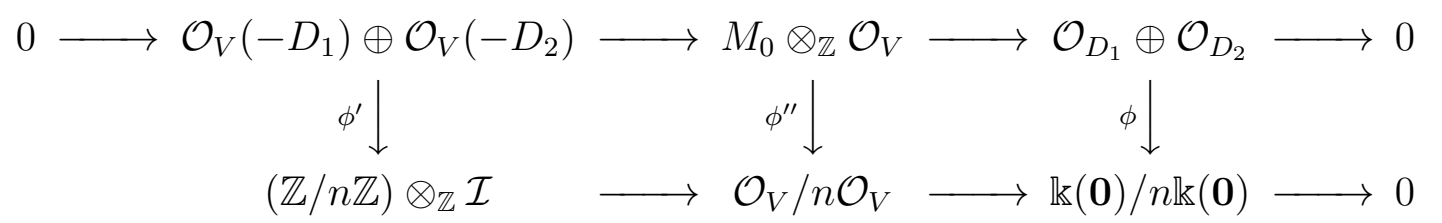

of exact sequences, where $\mathcal{I}$ is the defining ideal of $\mathbf{0}$, and the homomorphisms $\phi^{\prime}$ and $\phi^{\prime \prime}$ are also induced by

$$
\mathcal{O}_{V} \oplus \mathcal{O}_{V} \ni\left(\beta_{1}, \beta_{2}\right) \mapsto \beta_{1}+q \beta_{2} \in \mathcal{O}_{V}
$$


Here, $\phi^{\prime}$ is surjective, since $\mathcal{I}=\mathcal{O}_{V}\left(-D_{1}\right)+\mathcal{O}\left(-D_{2}\right)$ (cf. Remark 2.2.1) and since $\operatorname{gcd}(n, q)=1$. The kernel of $\phi^{\prime \prime}$ is just the image of the natural homomorphism $M \otimes_{\mathbb{Z}}$ $\mathcal{O}_{V} \rightarrow M_{0} \otimes \mathcal{O}_{V}$. Thus, the image of Res: $M \otimes_{\mathbb{Z}} \mathcal{O}_{V} \rightarrow \mathcal{O}_{D_{1}} \oplus \mathcal{O}_{D_{2}}$ is just the kernel of $\phi$. In particular, Res is surjective if and only if $p \nmid n$.

Assume that $p \mid n$. Then, $p \nmid q$ by $\operatorname{gcd}(n, q)=1$. Hence, the image of Res is also the kernel of

$$
\left.\mathcal{O}_{D_{1}} \oplus \mathcal{O}_{D_{2}} \ni\left(\alpha_{1}, \alpha_{2}\right) \mapsto \alpha_{1}\right|_{\mathbf{0}}+\left.\alpha_{2}\right|_{\mathbf{0}} \in \mathbb{k}(\mathbf{0}) .
$$

Thus, the image of Res is just $\mathcal{O}_{D}$, since we have a natural exact sequence $0 \rightarrow \mathcal{O}_{D} \rightarrow$ $\mathcal{O}_{D_{1}} \oplus \mathcal{O}_{D_{2}} \rightarrow \mathbb{k}(\mathbf{0}) \rightarrow 0$.

Proposition 2.11. Let $Y$ be a normal algebraic surface over an algebraically closed field $\mathbb{k}$ and let $\mu: M \rightarrow Y$ be the minimal resolution of singularities. Assume that any connected component of the $\mu$-exceptional locus $E$ is a linear chain of rational curves. Then, the following hold:

(1) The natural injection $\mu_{*} \Theta_{M / \mathbb{k}}(-\log E) \rightarrow \mu_{*} \Theta_{M / \mathbb{k}}$ is an isomorphism.

(2) $\mathrm{R}^{i} \mu_{*} \Theta_{M / \mathbb{k}}(-\log E)=0$ for all $i>0$.

(3) The direct image sheaf $\mu_{*} \Omega_{M / \mathbb{k}}^{1}$ is reflexive. In other words, $\left(\Omega_{Y / \mathbb{k}}^{1}\right)^{\vee \vee} \simeq \mu_{*} \Omega_{M / \mathbb{k}}^{1}$.

(4) The natural injection $\mu_{*} \Theta_{M / \mathbb{k}} \hookrightarrow \Theta_{Y / \mathbb{k}}$ is not an isomorphism if and only if $p \mid n$ and $q=n-1$.

Proof. Since the assertions are étale local on $Y$, we may assume that $Y$ is the toric surface $V=\mathbb{T}_{\mathrm{N}}(\boldsymbol{\sigma})$ by Theorem 2.6. Let $D_{1}, D_{2}, \mathbf{0}, \nu: U \rightarrow V$, and $G_{i}$ be as before. Then, the minimal resolution $M \rightarrow Y$ is just $U \rightarrow V$ and $E=G=\sum_{i=1}^{l} G_{i}$. We set $\widehat{G}=\sum_{i=0}^{l+1} G_{i}=G+G_{0}+G_{l+1}$. Since $G$ and $\widehat{G}$ are simple normal crossing divisors, we have a commutative diagram

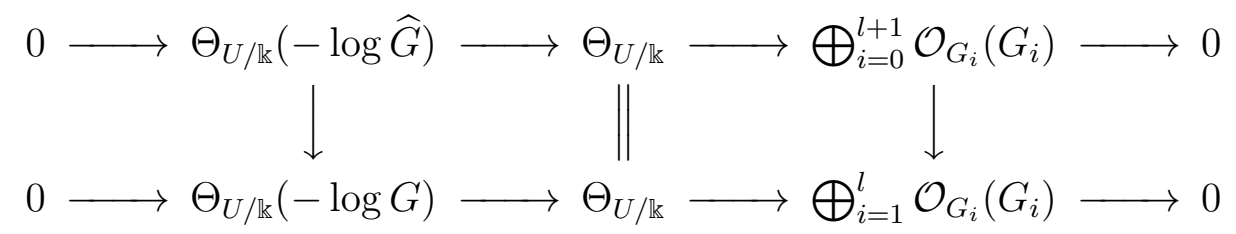

of exact sequences. The assertion (11) is derived from the bottom sequence by taking $\nu_{*}$, since $\nu_{*} \mathcal{O}_{G_{i}}\left(G_{i}\right)=\mathrm{H}^{0}\left(\mathbb{P}^{1}, \mathcal{O}\left(-b_{i}\right)\right)=0$ for $1 \leq i \leq l$. The commutative diagram above induces an exact sequence

$$
0 \rightarrow \Theta_{U / \mathbb{k}}(-\log \widehat{G}) \rightarrow \Theta_{U / \mathbb{k}}(-\log G) \rightarrow \mathcal{O}_{G_{0}}\left(G_{0}\right) \oplus \mathcal{O}_{G_{l+1}}\left(G_{l+1}\right) \rightarrow 0
$$

Here, $\Theta_{U / \mathbb{k}}(-\log \widehat{G}) \simeq \mathrm{N} \otimes_{\mathbb{Z}} \mathcal{O}_{U}$, since $\widehat{G}$ is the complement of the torus $\mathbb{T}_{\mathrm{N}}$ in $U=\mathbb{T}_{\mathrm{N}}(\triangle)$. Now, $\mathrm{R}^{i} \nu_{*} \mathcal{O}_{U}=0$ for $i>0$, since $V$ has only rational singularities (cf. Corollary 2.8.1). 
We have also $\mathrm{R}^{i} \nu_{*} \mathcal{O}_{G_{0}}\left(G_{0}\right)=\mathrm{R}^{i} \nu_{*} \mathcal{O}_{G_{l+1}}\left(G_{l+1}\right)=0$ for $i>0$, since $\nu$ induces isomorphisms $G_{0} \rightarrow D_{2}$ and $G_{l+1} \rightarrow D_{1}$. Thus, the assertion (2) is obtained by applying $\mathrm{R}^{i} \nu_{*}$ to the exact sequence ([I-10).

For the assertion (3), we consider the commutative diagram

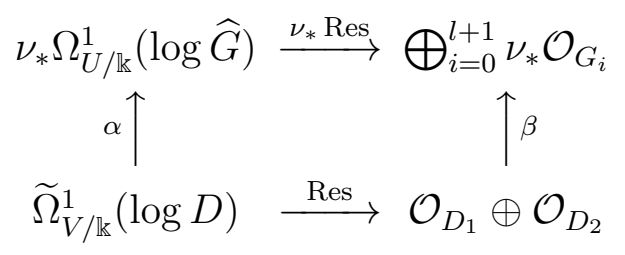

obtained by comparing the residue homomorphisms on $V$ and $U$. Here, $\alpha$ is an isomorphism, since $\widetilde{\Omega}_{V}^{1}(\log D) \simeq \mathrm{M} \otimes_{\mathbb{Z}} \mathcal{O}_{V}$ and $\Omega_{U}^{1}(\log \widehat{G}) \simeq \mathrm{M} \otimes_{\mathbb{Z}} \mathcal{O}_{U}$. The map $\beta$ is an isomorphism to $\nu_{*} \mathcal{O}_{E_{l+1}} \oplus \nu_{*} \mathcal{O}_{E_{0}}$. Thus, we have a homomorphism

$$
\gamma:\left(\Omega_{V / \mathbb{k}}^{1}\right)^{\vee \vee} \rightarrow \nu_{*} \Omega_{U / \mathbb{k}}^{1}
$$

as the induced homomorphism between the kernels of the top and bottom homomorphisms. Then, $\gamma$ is an isomorphism, since it is an isomorphism on $V \backslash\{\mathbf{0}\}$, the source is reflexive, and the target is torsion free. Hence, we have (33).

It remains to prove (44). Let $\mathcal{F}$ be the image of Res: $\widetilde{\Omega}_{V / \mathbb{k}}^{1}(\log D) \rightarrow \mathcal{O}_{D_{1}} \oplus \mathcal{O}_{D_{2}}$. Then, by (ㅍ-10) and by Lemma 2.9 , we have a commutative diagram

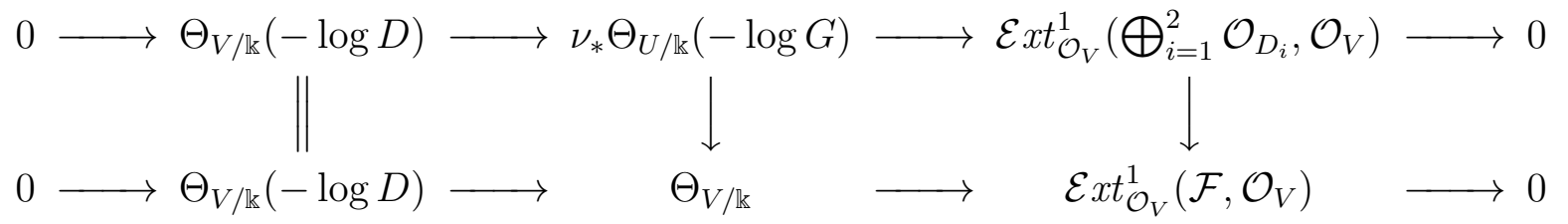

of exact sequences, in which the bottom one is obtained from

$$
0 \rightarrow\left(\Omega_{V / \mathbb{k}}^{1}\right)^{\vee \vee} \rightarrow \widetilde{\Omega}_{V / \mathbb{k}}^{1}(\log D) \rightarrow \mathcal{F} \rightarrow 0
$$

by taking $\mathcal{E} x t_{\mathcal{O}_{V}}^{i}\left(\bullet, \mathcal{O}_{V}\right)$. Hence, if $p \nmid n$, then $\mathcal{F}=\mathcal{O}_{D_{1}} \oplus \mathcal{O}_{D_{2}}$ by Lemma 2.10 and $\Theta_{V / \mathbb{k}} \simeq \nu_{*} \Theta_{U / \mathbb{k}}(-\log G)$ by the commutative diagram above. Thus, we may assume that $p \mid n$. Then, by Lemma $2.10, \mathcal{F}=\mathcal{O}_{D}$, and we obtain an exact sequence

$$
\begin{aligned}
\cdots \rightarrow \mathcal{E} x t_{\mathcal{O}_{V}}^{i}\left(\mathbb{k}(\mathbf{0}), \mathcal{O}_{V}\right) \rightarrow \mathcal{E} x t_{\mathcal{O}_{V}}^{i}\left(\mathcal{O}_{D_{1}}\right. & \left.\oplus \mathcal{O}_{D_{2}}, \mathcal{O}_{V}\right) \\
& \rightarrow \mathcal{E}^{i} t_{\mathcal{O}_{V}}^{i}\left(\mathcal{O}_{D}, \mathcal{O}_{V}\right) \rightarrow \mathcal{E} x t_{\mathcal{O}_{V}}^{i+1}\left(\mathbb{k}(\mathbf{0}), \mathcal{O}_{V}\right) \rightarrow \cdots
\end{aligned}
$$

It is enough to determine when the cokernel of

$$
\mathcal{E} x t_{\mathcal{O}_{V}}^{1}\left(\mathcal{O}_{D_{1}} \oplus \mathcal{O}_{D_{2}}, \mathcal{O}_{V}\right) \rightarrow \mathcal{E} x t_{\mathcal{O}_{V}}^{1}\left(\mathcal{O}_{D}, \mathcal{O}_{V}\right)
$$

is not zero. By a standard argument, we see that the cokernel is isomorphic to that of the homomorphism $\mathcal{O}_{V}\left(D_{1}\right) \oplus \mathcal{O}_{V}\left(D_{2}\right) \rightarrow \mathcal{O}_{V}(D)$ induced from the natural inclusions $\mathcal{O}_{V}\left(D_{1}\right) \hookrightarrow \mathcal{O}_{V}(D)$ and $\mathcal{O}_{V}\left(D_{2}\right) \hookrightarrow \mathcal{O}_{V}(D)$. Now, $\mathrm{H}^{0}\left(V, \mathcal{O}_{V}(D)\right)$ is generated by the 
rational functions $\varphi$ on $V$ such that $\operatorname{div}(\varphi)+D \geq 0$. Hence, $\mathrm{H}^{0}\left(V, \mathcal{O}_{V}(D)\right)$ is generated by the following monomials $\mathrm{x}_{1}^{a_{1}} \mathrm{x}_{2}^{a_{2}}$ as an $\mathrm{H}^{0}\left(V, \mathcal{O}_{V}\right)$-submodule of $\mathbb{k}[\mathrm{M}]=\mathrm{H}^{0}\left(\mathbb{T}_{\mathrm{N}}, \mathcal{O}\right)$ :

- $a_{1}$ and $a_{2}$ are integers with $a_{1}+q a_{2} \equiv 0 \bmod n$.

- $a_{1} \geq-1$ and $a_{2} \geq-1$ (cf. (프-2 $)$ ).

Similarly, $\mathrm{H}^{0}\left(V, \mathcal{O}_{V}\left(D_{1}\right)\right)+\mathrm{H}^{0}\left(V, \mathcal{O}_{V}\left(D_{2}\right)\right)$ is generated by the following monomials $\mathrm{x}_{1}^{b_{1}} \mathbf{x}_{2}^{b_{2}}$ as an $\mathrm{H}^{0}\left(V, \mathcal{O}_{V}\right)$-submodule of $\mathbb{k}[\mathrm{M}]$ :

- $b_{1}$ and $b_{2}$ are integers with $b_{1}+q b_{2} \equiv 0 \bmod n$.

- $b_{1} \geq-1$ and $b_{2} \geq-1$, but $\max \left\{b_{1}, b_{2}\right\} \geq 0$.

Therefore, the cokernel is not zero if and only if the monomial $\mathrm{x}_{1}^{-1} \mathrm{x}_{2}^{-1}$ is contained in $\mathrm{H}^{0}\left(V, \mathcal{O}_{V}(D)\right)$. This is just the case where $q=n-1$. Thus (4) is proved, and we have finished the proof of Proposition 2.11,

Remark 2.11.1. If the injection of the assertion (4) is an isomorphism, then $\mu$ is an "equivariant resolution of singularities" in the sense of Hironaka. Hence, for toric singularities of type $(n, q=n-1)$, equivariant resolutions do exist if and only if $p \nmid n$ (cf. [53, Theorem] and an example of characteristic two in [5, page 345]).

Corollary 2.12. In the situation of Proposition 2.11, one has an isomorphism

$$
\mathrm{H}^{2}\left(M, \Theta_{M / \mathbb{k}}(-\log E)\right) \stackrel{\simeq}{\rightarrow} \mathrm{H}^{2}\left(Y, \Theta_{Y / \mathbb{k}}\right) .
$$

Proof. Since $\Theta_{Y / \mathbb{k}}$ is the double-dual of $\mu_{*} \Theta_{M / \mathbb{k}}(-\log E)$, there is an exact sequence

$$
0 \rightarrow \mu_{*} \Theta_{M / \mathbb{k}}(-\log E) \rightarrow \Theta_{Y / \mathbb{k}} \rightarrow \mathcal{G} \rightarrow 0
$$

for a coherent sheaf $\mathcal{G}$ with $\operatorname{dim} \operatorname{Supp} \mathcal{G} \leq 0$, which induces an isomorphism

$$
\iota_{1}: \mathrm{H}^{2}\left(Y, \mu_{*} \Theta_{M / \mathbb{k}}(-\log E)\right) \simeq \mathrm{H}^{2}\left(Y, \Theta_{Y / \mathbb{k}}\right) .
$$

On the other hand, since $\mathrm{R}^{i} \mu_{*} \Theta_{M / \mathbb{k}}(-\log E)=0$ for all $i>0$ by Proposition 2.11,(2), the Leray spectral sequence for $\mu$ induces an isomorphism

$$
i_{2}: \mathrm{H}^{2}\left(X, \mu_{*} \Theta_{M / \mathbb{k}}(-\log E)\right) \simeq \mathrm{H}^{2}\left(M, \Theta_{M / \mathbb{k}}(-\log E)\right) .
$$

Therefore, we obtain the claimed isomorphism as $i_{1} \circ i_{2}^{-1}$.

\section{TORIC SINGULARITY OF CLASS T}

In this section, we introduce the notion of toric singularity of class $\mathrm{T}$ and study its properties. We first discuss some invariants arising from toric singularities of class $\mathrm{T}$ : The results here are already known in papers such as [54], [37], [26], [52], [38], [32], but we shall give a self-contained proof. Tables 1 and 2 obtained here are used in some calculations in Sections 6 and 7 . Second, in Theorem 3.8 below, we shall construct a special smoothing 
(deformation) of toric singularities of class $\mathrm{T}$, which plays an important role in producing new surfaces.

Definition 3.1. Let $X$ be a normal algebraic surface defined over $\mathbb{k}$ and $x$ a closed point. The germ $(X, x)$ (in the étale topology) is said to be a "toric singularity of type $(n, q)$ " if the formal completion of $\mathcal{O}_{X, x}$ is isomorphic to the formal completion of $\mathcal{O}_{V, \mathbf{0}}$ for an affine toric surface $V$ of type $(n, q)$ (cf. Definition 2.2 ) over $\mathbb{k}$ and the zero-dimensional orbit $\mathbf{0 .}$

Remark 3.1.1. Note that by [2, Corollary 2.6], the condition in Definition 3.1] is equivalent to the existence of a common étale neighborhood of $(X, x)$ and $(V, \mathbf{0})$.

Remark 3.1.2. By Theorem 2.7, $(X, x)$ is a toric singularity if and only if the exceptional locus of the minimal resolution is a linear chain of smooth rational curves.

Definition 3.2. Let $\mathcal{T}_{\text {DNA }}$ be the set of triples $(d, n, a)$ of positive integers with $n>a$ and $\operatorname{gcd}(n, a)=1$. A two-dimensional surface singularity is said to be of type $T(d, n, a)$ for a triplet $(d, n, a) \in \mathcal{T}_{\text {DNA }}$ if it is a toric singularity of type $\left(d n^{2}, d n a-1\right)$. The singularities of "class $\mathrm{T}$ " are the singularities of type $T(d, n, a)$ for all $(d, n, a) \in \mathcal{T}_{\mathrm{DNA}}$ (cf. [26, Proposition 3.10], [38, §4]).

Remark 3.2.1. The definition of class $\mathrm{T}$ in [26] is different from ours. Our definition of class $\mathrm{T}$ corresponds to that of non-Gorenstein class $\mathrm{T}$ in [26].

Before going to the study of toric singularities of class $\mathrm{T}$, we prepare some invariants arising from each element of $\mathcal{T}_{\text {DNA }}$. Let $(d, n, a)$ be a triplet in $\mathcal{T}_{\text {DNA }}$. We can define positive integers $l, b_{1}, \ldots, b_{l}$ by the property that $l \geq 1, b_{i} \geq 2$ for all $1 \leq i \leq l$, and $d n^{2} /(d n a-1)=\left[b_{1}, \ldots, b_{l}\right]$. Then, $\left(b_{1}, \ldots, b_{l}\right) \neq(2,2, \ldots, 2)$; for otherwise, $d n^{2}=d n a$ contradicting $n>a$. By Lemma 2.3 , we can define also non-negative integers $p_{i}$ and $q_{i}$ for $0 \leq i \leq l+1$ by the following properties:

- $p_{0}=0<p_{1}=1<p_{2}<\cdots<p_{l}<p_{l+1}=d n^{2}$.

- $q_{0}=d n^{2}>q_{1}=d n a-1>q_{2}>\cdots>q_{l}=1>q_{l+1}=0$

- $p_{i-1}+p_{i+1}=b_{i} p_{i}$ and $q_{i-1}+q_{i+1}=b_{i} q_{i}$ for all $1 \leq i \leq l$.

We set $r_{i}:=\left(p_{i}+q_{i}\right) /(d n)$ for $0 \leq i \leq l+1$. Then, $r_{0}=r_{l+1}=n$ and $r_{1}=a$. Moreover, we have:

Lemma 3.3. (1) $r_{i}$ is a positive integer with $1 \leq r_{i}<n$ for all $1 \leq i \leq l$.

(2) $r_{i} \equiv a p_{i} \equiv-a q_{i} \bmod n$ for all $1 \leq i \leq l$.

(3) $r_{l}=n-a$, equivalently, $p_{l}=d n(n-a)-1$. 
Proof. The assertions (11) and (2) follow from the convexity $r_{i-1}+r_{i+1}=b_{i} r_{i}$ and $p_{i} q_{1} \equiv q_{i}$ $\bmod d n^{2}$ for $1 \leq i \leq l$ (cf. Lemma 2.3.(5) ). The last assertion (3) is a consequence of the previous two assertions.

Definition 3.4. For $(d, n, a) \in \mathcal{T}_{\mathrm{DNA}}$, we define:

$$
\begin{gathered}
B(d, n, a):=\left(b_{1}, b_{2}, \ldots, b_{l}\right), \quad P(d, n, a):=\left(p_{1}, p_{2}, \ldots, p_{l}\right), \quad Q(d, n, a):=\left(q_{1}, q_{2}, \ldots, q_{l}\right), \\
R(d, n, a):=\left(r_{1}, r_{2}, \ldots, r_{l}\right), \quad C(d, n, a):=\left(c_{1}, c_{2}, \ldots, c_{l}\right) \quad \text { where } \quad c_{i}:=1-r_{i} / n, \\
\delta(d, n, a):=\sum_{i=1}^{l} b_{i}-(2 l+1), \quad \text { and } \quad l(d, n, a):=l .
\end{gathered}
$$

The following characterization of toric singularities of class $\mathrm{T}$ is well-known:

Lemma 3.5 ([37, Proposition 5.9]). Let $(X, x)$ be a normal surface singularity such that the exceptional locus of the minimal resolution $\mu: M \rightarrow(X, x)$ of singularity is a linear chain of smooth rational curves. Then, the following two conditions are mutually equivalent:

(1) $\Delta^{2}$ is a negative integer for the effective $\mathbb{Q}$-divisor $\Delta=\mu^{*}\left(K_{X}\right)-K_{M}$.

(2) $(X, x)$ is a toric surface singularity of class $T$.

Moreover, if $(X, x)$ is a singularity of type $T(d, n, a)$, then $\Delta^{2}=-\delta(d, n, a)$, and $\Delta=$ $\sum_{i=1}^{l} c_{i} E_{i}$ for the linear chain $E_{1}+\cdots+E_{l}$ of smooth rational curves and for $C(d, n, a)=$ $\left(c_{1}, \ldots, c_{l}\right)$.

Proof. By Theorem 2.7, we may assume that $X$ is a toric surface $V$ of type $(n, q)$ for some positive integers $n, q$ with $n>q$ and $\operatorname{gcd}(n, q)=1$. Thus, we can use the description of the minimal resolution $\nu: U \rightarrow V$ of the toric surface given in Section 2. Note that $\nu^{*}\left(K_{V}+D_{1}+D_{2}\right)=K_{U}+\sum_{i=0}^{l+1} G_{i} \sim 0$. Thus, we have

$$
\Delta=\nu^{*}\left(K_{V}\right)-K_{U}=\sum_{i=0}^{l+1} G_{i}-\nu^{*}\left(D_{1}+D_{2}\right)=\sum_{i=1}^{l}\left(1-\frac{p_{i}+q_{i}}{n}\right) G_{i}
$$

for integers $p_{i}, q_{i}$ in Lemma 2.3, by (II-6) in Remark 2.4.1. In particular, if $(n, q)=$ $\left(d m^{2}, d m a-1\right)$ for a triplet $(d, m, a) \in \mathcal{T}_{\mathrm{DNA}}$, then we have $\Delta=\sum_{i=1}^{l} c_{i} G_{i}$ for $C(d, m, a)=$ $\left(c_{1}, \ldots, c_{m}\right)$. On the other hand,

$$
\Delta G_{i}=-K_{U} G_{i}=2+G_{i}^{2}=2-b_{i}
$$

for all $1 \leq i \leq l$ by adjunction, where $n / q=\left[b_{1}, \ldots, b_{l}\right]$. Hence,

$$
\Delta^{2}=\sum_{i=1}^{l}\left(1-\frac{p_{i}+q_{i}}{n}\right)\left(2-b_{i}\right)
$$

and it is an integer if and only if

$$
\sum_{i=1}^{l}\left(p_{i}+q_{i}\right)\left(2-b_{i}\right) \equiv 0 \bmod n .
$$


Since $b_{i}\left(p_{i}, q_{i}\right)=\left(p_{i-1}, q_{i-1}\right)+\left(p_{i+1}, q_{i+1}\right)$ (cf. Lemma 2.3), we have

$$
\begin{aligned}
& \sum_{i=1}^{l}\left(p_{i}+q_{i}\right)\left(2-b_{i}\right)=2 \sum_{i=1}^{l}\left(p_{i}+q_{i}\right)-\sum_{i=1}^{l}\left(p_{i-1}+q_{i-1}\right)-\sum_{i=1}^{l}\left(p_{i+1}+q_{i+1}\right) \\
& =p_{1}+p_{l}+q_{1}+q_{l}-\left(p_{0}+p_{l+1}+q_{0}+q_{l+1}\right)=q+q^{\prime}+2-2 n,
\end{aligned}
$$

where $0<q^{\prime}<n$ with $q q^{\prime} \equiv 1 \bmod n$ (cf. Lemma 2.3.(5) ). Thus, $\Delta^{2} \in \mathbb{Z}$ if and only if $q+q^{\prime}+2 \equiv 0 \bmod n$. Since $\operatorname{gcd}(n, q)=1$, this condition is equivalent to

$$
(q+1)^{2}=q^{2}+2 q+1 \equiv q\left(q+2+q^{\prime}\right) \equiv 0 \bmod n .
$$

By considering the prime factorization, we see that this is also equivalent to either

(i) $n=q+1$, or

(ii) $n=d m^{2}$ and $q+1=d m a$ for some $(d, m, a) \in \mathcal{T}_{\text {DNA }}$.

In case (ii),$\left(b_{1}, \ldots, b_{l}\right)=(2,2, \ldots, 2)$ and $\Delta^{2}=0$. In case (iii), we have $q+q^{\prime}+2=d m^{2}$ by Lemma 3.3 , (3) , and

$$
\Delta^{2}=\sum_{i=1}^{l}\left(2-b_{i}\right)-\frac{1}{d m^{2}}\left(q+q^{\prime}+2-2 d m^{2}\right)=2 l+1-\sum_{i=1}^{l} b_{i}=-\delta(d, m, a)<0 .
$$

Thus, we are done.

Corollary 3.5.1. Let $X$ be a normal projective surface whose non-Gorenstein singularities are toric singularities of class $T$. Then, $K_{X}^{2}$ is an integer.

Lemma 3.6 (cf. [26, Proposition 3.11]). For a triplet $(d, n, a) \in \mathcal{T}_{\mathrm{DNA}}$, either $b_{1} \geq 3$ or $b_{l} \geq 3$ holds for $B(d, n, a)=\left(b_{1}, \ldots, b_{l}\right)$. Assume that $b_{1} \geq 3$ and $b_{l} \geq 3$. Then, $(d, n, a)=$ $(l, 2,1)$. Here, $B(1,2,1)=(4), B(2,2,1)=(3,3)$, and $B(l, 2,1)=(3,2, \ldots, 2,3)$ for $l \geq 3$. Moreover, for all $1 \leq i \leq l$,

$$
\left(p_{i}, q_{i}, r_{i}, c_{i}\right)=(2 i-1,2(l-i)+1,1,1 / 2) .
$$

Proof. Assume first that $l=1$. Then $d n^{2}=b_{1}(d n a-1)$ and $\operatorname{gcd}\left(d n^{2}, d n a-1\right)=1$ imply that $d n^{2}=b_{1}$ and $d n a=2$. Hence $(d, n, a)=(1,2,1)$ and $b_{1}=4$. In this case, $\left(p_{1}, q_{1}, r_{1}, c_{1}\right)=(1,1,1,1 / 2)$.

Thus, we may assume that $l \geq 2$. Since $p_{l}=d n(n-a)-1$ by Lemma 3.3, we have

$$
\frac{n}{b_{1}}+\frac{1}{d n} \leq a<\frac{n}{b_{1}-1}+\frac{1}{d n} \text { and } \quad \frac{n}{b_{l}}+\frac{1}{d n} \leq n-a<\frac{n}{b_{l}-1}+\frac{1}{d n}
$$

from $0 \leq q_{2}=b_{1} q_{1}-q_{0}<q_{1}$ and $0 \leq p_{l-1}=b_{l} p_{l}-p_{l+1}<p_{l}$. In particular,

$$
\frac{1}{b_{1}}+\frac{1}{b_{l}}+\frac{2}{d n^{2}} \leq 1
$$

and hence, $b_{1} \geq 3$ or $b_{l} \geq 3$ holds. Assume that $b_{1} \geq 3$ and $b_{l} \geq 3$. Then,

$$
\frac{n}{2}-\frac{1}{d n} \leq n\left(1-\frac{1}{b_{l}-1}\right)-\frac{1}{d n}<a<\frac{n}{b_{1}-1}+\frac{1}{d n} \leq \frac{n}{2}+\frac{1}{d n}
$$


by (III-1). Since $n \geq 2$ and $a \in \mathbb{Z}$, we have $a=n / 2$. Thus, $(n, a)=(2,1)$, since $\operatorname{gcd}(n, a)=1$. Therefore, $q_{0}=p_{l+1}=4 d, q_{1}=p_{l}=2 d-1$, and hence, $r_{0}=r_{l+1}=2$. For $1 \leq i \leq l$, we have $r_{i}=1$, since $0<r_{i}<n=2$ by Lemma 3.3.(1). Thus, $c_{i}=1 / 2$ and $p_{i}+q_{i}=2 d$ for all $1 \leq i \leq l$. As a consequence,

$$
b_{i}=\frac{r_{i-1}+r_{i+1}}{r_{i}}= \begin{cases}2, & \text { if } 1<i<l \\ 3, & \text { if } i=1 \text { or } i=l .\end{cases}
$$

The equalities $\left(p_{i}, q_{i}\right)=(2 i-1,2(d-i)+1)$ are shown by induction using $p_{i-1}+p_{i+1}=b_{i} p_{i}$ and $q_{i-1}+q_{i+1}=b_{i} q_{i}$ with the initial values $p_{1}=q_{l}=1$ and $p_{0}=q_{l+1}=0$.

If $(d, n, a) \in \mathcal{T}_{\text {DNA }}$, then $(d, n, n-a),(d, 2 n-a, n),(d, n+a, a) \in \mathcal{T}_{\text {DNA }}$. Thus, we have three maps $\boldsymbol{i}, \boldsymbol{t}_{L}, \boldsymbol{t}_{R}: \mathcal{T}_{\mathrm{DNA}} \rightarrow \mathcal{T}_{\mathrm{DNA}}$ defined by

$$
\boldsymbol{i}(d, n, a)=(d, n, n-a), \quad \boldsymbol{t}_{L}(d, n, a)=(d, 2 n-a, n), \quad \boldsymbol{t}_{R}(d, n, a)=(d, n+a, a) .
$$

Thus, $\boldsymbol{i}$ is an involution, and $\boldsymbol{t}_{L}=\boldsymbol{i} \circ \boldsymbol{t}_{R} \circ \boldsymbol{i}$. Concerning these maps, we have:

Lemma 3.7 (cf. [54, (2.8.2)], [26, Proposition 3.11], [52, Lemma 3.4], [38, Theorem 17], [32, Theorem 15]). Let $(d, n, a)$ be a triplet in $\mathcal{T}_{\mathrm{DNA}}$ with $B(d, n, a)=\left(b_{1}, b_{2}, \ldots, b_{l}\right)$ and $R(d, n, a)=\left(r_{1}, r_{2}, \ldots, r_{l}\right)$. Then, the following hold:

$$
\begin{aligned}
B(d, 2 n-a, n) & =\left(2, b_{1}, \ldots, b_{l-1}, b_{l}+1\right), & & \delta(d, 2 n-a, n)=\delta(d, n, a)+1, \\
R(d, 2 n-a, n) & =\left(n=r_{1}+r_{l}, r_{1}, \ldots, r_{l}\right), & l(d, 2 n-a, n) & =l(d, n, a)+1, \\
B(d, n+a, a) & =\left(b_{1}+1, b_{2}, \ldots, b_{l}, 2\right), & & \delta(d, n+a, a)=\delta(d, n, a)+1, \\
R(d, n+a, a) & =\left(r_{1}, \ldots, r_{l}, n=r_{1}+r_{l}\right), & l(d, n+a, a) & =l(d, n, a)+1 .
\end{aligned}
$$

Proof. We set

$$
P(d, n, a)=\left(p_{1}, \ldots, p_{l}\right), \quad Q(d, n, a)=\left(q_{1}, \ldots, q_{l}\right), \quad \text { and } \quad C(d, n, a)=\left(c_{1}, \ldots, c_{l}\right) .
$$

Concerning the map $\boldsymbol{i}:(d, n, a) \mapsto(d, n, n-a)$, we have

$$
\begin{array}{rlrl}
B(d, n, n-a) & =\left(b_{l}, b_{l-1}, \ldots, b_{1}\right), & & \delta(d, n, n-a)=\delta(d, n, a), \\
P(d, n, n-a)=\left(p_{l}, p_{l-1}, \ldots, p_{1}\right), & & Q(d, n, n-a)=\left(q_{l}, q_{l-1}, \ldots, q_{1}\right), \\
R(d, n, n-a)=\left(r_{l}, r_{l-1}, \ldots, r_{1}\right), & & C(d, n, n-a)=\left(c_{l}, c_{l-1}, \ldots, c_{1}\right),
\end{array}
$$

by $p_{l}=d n(n-a)-1$ (cf. Lemma 3.3.(3) $)$. Hence, the equalities for $\boldsymbol{t}_{L}(d, n, a)=$ $(d, 2 n-a, n)$ are derived from those for $\boldsymbol{t}_{R}(d, n, a)=(d, n+a, a)$ by $\boldsymbol{t}_{L}=\boldsymbol{i} \circ \boldsymbol{t}_{R} \circ \boldsymbol{i}$. Thus, it is enough to prove the equalities for $(d, n+a, a)$. We set

$$
\left(p_{0}^{\prime}, q_{0}^{\prime}\right):=\left(0, d(n+a)^{2}\right) \quad \text { and } \quad\left(p_{l+2}^{\prime}, q_{l+2}^{\prime}\right):=\left(d(n+a)^{2}, 0\right)
$$




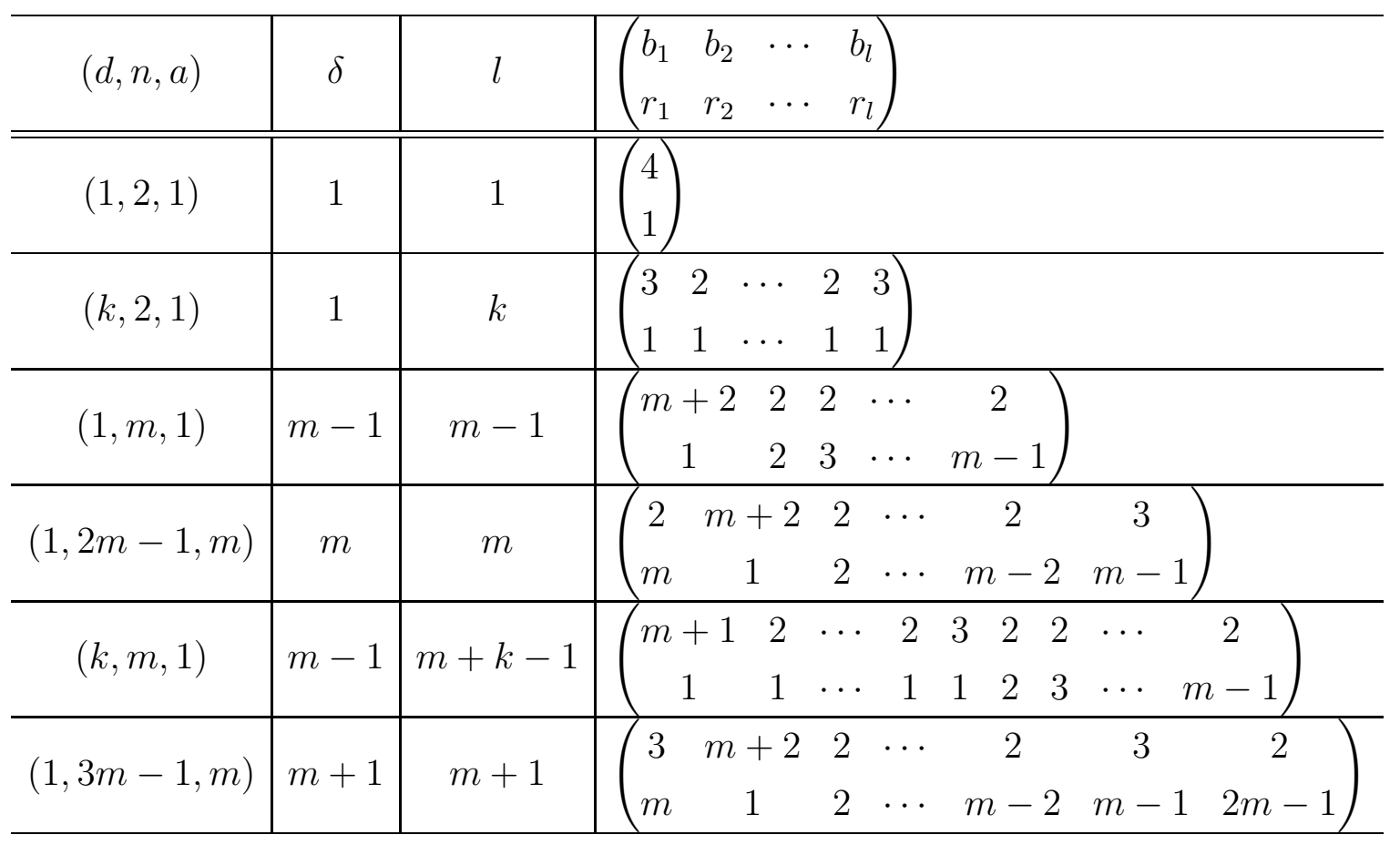

(Here, $k \geq 2$ and $m \geq 3$ )

TABLE 1. Invariants related to singularities of class T, Part I

and for $1 \leq i \leq l+1$, we set

$$
\left(p_{i}^{\prime}, q_{i}^{\prime}\right):=\frac{1}{d n^{2}}\left(p_{i}, q_{i}\right)\left(\begin{array}{cc}
d n(n+a)-1 & 1 \\
-1 & d n(n+a)+1
\end{array}\right) .
$$

Then, $p_{i}^{\prime}$ and $q_{i}^{\prime}$ are positive integers by Lemma 3.3.(2), and we have

$$
\left(p_{i-1}^{\prime}, q_{i-1}^{\prime}\right)+\left(p_{i+1}^{\prime}, q_{i+1}^{\prime}\right)= \begin{cases}\left(b_{1}+1\right)\left(p_{1}^{\prime}, q_{1}^{\prime}\right), & \text { for } i=1, \\ b_{i}\left(p_{i}^{\prime}, q_{i}^{\prime}\right), & \text { for } 2 \leq i \leq l, \\ 2\left(p_{l+1}^{\prime}, q_{l+1}^{\prime}\right), & \text { for } i=l+1,\end{cases}
$$

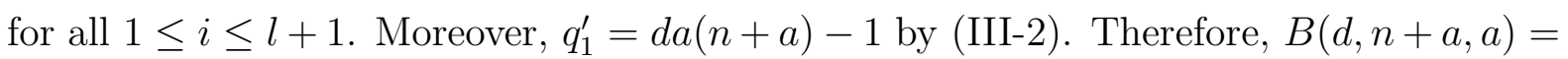
$\left(b_{1}+1, b_{2}, \ldots, b_{l}, 2\right), P(d, n+a, a)=\left(p_{1}^{\prime}, p_{2}^{\prime}, \ldots, p_{l+1}^{\prime}\right)$, and $Q(d, n+a, a)=\left(q_{1}^{\prime}, q_{2}^{\prime}, \ldots, q_{l+1}^{\prime}\right)$. In particular,

$$
\delta(d, n+a, a)=3+\sum_{i=1}^{l} b_{i}-(2(l+1)+1)=\delta(d, n+a, a)+1 .
$$

By (III-2), we have

$$
\frac{p_{i}^{\prime}+q_{i}^{\prime}}{d(n+a)}=\frac{d n(n+a)\left(p_{i}+q_{i}\right)}{d(n+a) \cdot d n^{2}}=\frac{p_{i}+q_{i}}{d n}=r_{i}
$$

for all $1 \leq i \leq l+1$. Hence, $R(d, n+a, a)=\left(r_{1}, \ldots, r_{l}, n=r_{1}+r_{l}\right)$. Thus, we are done. 


\begin{tabular}{|c|c|c|c|}
\hline$(d, n, a)$ & $\delta$ & $l$ & $\left(\begin{array}{llll}b_{1} & b_{2} & \cdots & b_{l} \\
r_{1} & r_{2} & \cdots & r_{l}\end{array}\right)$ \\
\hline$(1,11,3)$ & 5 & 5 & $\left(\begin{array}{lllll}4 & 5 & 3 & 2 & 2 \\
3 & 1 & 2 & 5 & 8\end{array}\right)$ \\
\hline$(1,19,5)$ & 7 & 7 & $\left(\begin{array}{ccccccc}4 & 7 & 2 & 2 & 3 & 2 & 2 \\
5 & 1 & 2 & 3 & 4 & 9 & 14\end{array}\right)$ \\
\hline$(1,19,13)$ & 8 & 8 & $\left(\begin{array}{cccccccc}2 & 2 & 9 & 2 & 2 & 2 & 2 & 4 \\
13 & 7 & 1 & 2 & 3 & 4 & 5 & 6\end{array}\right)$ \\
\hline$(3,23,4)$ & 8 & 10 & $\left(\begin{array}{cccccccccc}6 & 5 & 2 & 3 & 2 & 3 & 2 & 2 & 2 & 2 \\
4 & 1 & 1 & 1 & 2 & 3 & 7 & 11 & 15 & 19\end{array}\right)$ \\
\hline$(1,25,17)$ & 10 & 10 & $\left(\begin{array}{cccccccccc}2 & 2 & 11 & 2 & 2 & 2 & 2 & 2 & 2 & 4 \\
17 & 9 & 1 & 2 & 3 & 4 & 5 & 6 & 7 & 8\end{array}\right)$ \\
\hline$(1,35,6)$ & 10 & 10 & $\left(\begin{array}{cccccccccc}6 & 8 & 2 & 2 & 2 & 3 & 2 & 2 & 2 & 2 \\
6 & 1 & 2 & 3 & 4 & 5 & 11 & 17 & 23 & 29\end{array}\right)$ \\
\hline$(1,63,34)$ & 11 & 11 & $\left(\begin{array}{ccccccccccc}2 & 7 & 7 & 2 & 2 & 3 & 2 & 2 & 2 & 2 & 3 \\
34 & 5 & 1 & 2 & 3 & 4 & 9 & 14 & 19 & 24 & 29\end{array}\right)$ \\
\hline$(1,252,145)$ & 13 & 13 & $\left(\begin{array}{ccccccccccccc}2 & 4 & 6 & 2 & 6 & 2 & 4 & 2 & 2 & 2 & 3 & 2 & 3 \\
145 & 38 & 7 & 4 & 1 & 2 & 3 & 10 & 17 & 24 & 31 & 69 & 107\end{array}\right.$ \\
\hline
\end{tabular}

TABLE 2. Invariants related to singularities of class T, Part II

Corollary 3.7.1 (cf. [26, Proposition 3.11], [38, Theorem 17], [32, Proposition 20]). Any element $(d, n, a)$ of $\mathcal{T}_{\mathrm{DNA}}$ is obtained from $(d, 2,1)$ by a successive compositions of maps $\boldsymbol{t}_{L}$ and $\boldsymbol{t}_{R}$. The number of the compositions equals $\delta(d, n, a)-1=l(d, n, a)-d$. In particular, $\sum_{i=1}^{l} b_{i}=3 l+2-d$.

Proof. If $b_{1} \geq 3$ and $b_{l} \geq 3$, then $(d, n, a)=(d, 2,1), l(d, 2,1)=d$, and $\delta(d, 2,1)=1$ by Lemma 3.6. If $b_{1}=2$, then $b_{l} \geq 3$ by Lemma 3.6, and $(d, n, a)=\boldsymbol{t}_{L}(d, a, n-2 a)$ with $l(d, n, a)=l(d, a, n-2 a)+1$ and $\delta(d, n, a)=\delta(d, a, n-2 a)+1$ by Lemma 3.7. Similarly, if $b_{l}=2$, then $b_{1} \geq 3$, and $(d, n, a)=\boldsymbol{t}_{R}(d, n-a, a)$ with $l(d, n, a)=l(d, n-a, a)+1$ and $\delta(d, n, a)=\delta(d, n-a, a)+1$. Hence, we are done by induction on $l(d, n, a)$.

In Tables 1 and 2, we list $\delta=\delta(d, n, a), l=l(d, n, a), B(d, n, a)$, and $R(d, n, a)$ for typical elements $(d, n, a) \in \mathcal{T}_{\text {DNA }}$, some of which are used later. For the numbers $c_{i}$, we have: 
Corollary 3.7.2 (cf. [32, Corollary 17]). Let $(d, n, a)$ be a triplet in $\mathcal{T}_{\mathrm{DNA}}$ with $B(d, n, a)=$ $\left(b_{1}, \ldots, b_{l}\right), R(d, n, a)=\left(r_{1}, \ldots, r_{l}\right)$, and $C(d, n, a)=\left(c_{1}, \ldots, c_{l}\right)$. If $c_{i} \leq 1 / 2$ (or equivalently, $\left.r_{i} / n \geq 1 / 2\right)$ for some $i$ and if $n>2$, then either $b_{j}=2$ for all $j \leq i$ or $b_{k}=2$ for all $k \leq i$.

Proof. Assume the contrary. Then, $b_{j} \geq 3$ and $b_{k} \geq 3$ for some $j \leq i \leq k$. By Lemma 3.6, we have $b_{1}=2$ or $b_{l}=2$. Thus, we may assume that $b_{1} \geq 3$ and $b_{l}=2$. In particular, we may put $j=1$. Then, $(d, n, a)=\boldsymbol{t}_{R}(d, n-a, a)$, and

$$
B(d, n-a, a)=\left(b_{1}-1, b_{2}, \ldots, b_{l-1}\right) \quad \text { and } \quad R(d, n-a, a)=\left(r_{1}, r_{2}, \ldots, r_{l-1}\right)
$$

by Lemma 3.7. In particular,

$$
c_{i}^{\prime}=1-r_{i}^{\prime} /(n-a)<1-r_{i} / n=c_{i} \leq 1 / 2,
$$

where $C(d, n-a, a)=\left(c_{1}^{\prime}, \ldots, c_{l-1}^{\prime}\right)$. Hence, in order to derive a contradiction, we may assume that $k=l-1$.

If $b_{1}-1 \geq 3$, then $n-a=2$ and $r_{i}=1$ by Lemma 3.6, this is a contradiction, since $r_{i} / n \leq 1 / 3$. Thus, $b_{1}=3$ and $(d, n-a, a)=\boldsymbol{t}_{L}(d, a, 3 a-n)$ by Lemma 3.7. In particular, $2 a<n<3 a$. Moreover,

$$
B(d, a, 3 a-n)=\left(b_{2}, \ldots, b_{l-2}, b_{l-1}-1\right) \quad \text { and } \quad R(d, a, 3 a-n)=\left(r_{2}, \ldots, r_{l-1}\right) .
$$

Thus, $r_{i}<a$ by Lemma 3.3 applied to $(d, a, 3 a-n)$, and hence, $r_{i} / n<a / n<1 / 2$; this is a contradiction.

Finally in Section [3, we shall prove:

Theorem 3.8. Let $\Lambda$ be a complete discrete valuation ring or a field. Let $V$ be a toric $\Lambda$-scheme of type $\left(d n^{2}, d n a-1\right)$ (cf. Definition 2.2) for some positive integers $d, n$, a with $n>a$ and $\operatorname{gcd}(n, a)=1$. Then, there exist a flat family $\mathcal{V} \rightarrow T$ of normal affine surfaces over an open subset $T$ of the affine line $\mathbb{A}_{\Lambda}^{1}$ and a section $\sigma$ : Spec $\Lambda \rightarrow T$ satisfying the following conditions:

(1) $\mathcal{V} \times_{T, \sigma} \operatorname{Spec} \Lambda \simeq V$.

$(2) \mathcal{V} \rightarrow T$ is smooth over $T \backslash \sigma(\operatorname{Spec} \Lambda)$.

$(3) \mathcal{V}$ is normal, and $r K_{\mathcal{V}}$ is Cartier with $\left.\mathcal{O}_{\mathcal{V}}\left(r K_{\mathcal{V}}\right)\right|_{V} \simeq \mathcal{O}_{V}\left(r K_{V}\right)$ for any integer $r$ divisible by $n$.

Remark 3.8.1. Our idea of the proof of Theorem 3.8 is taken from the proof of [40, Proposition 4.19], which treats a special case. However, the proof of Proposition 4.19 contains an error when $p \mid n$. The error is corrected by the present proof. 
Remark 3.8.2. Roughly speaking, when $\Lambda$ is a field, Theorem 3.8 asserts that a toric singularity of class T has a "Q্Q-Gorenstein smoothing" (cf. [26]). The proof of Theorem 3.8 is essentially the same as in the proof of [26, Proposition 3.10] when $\Lambda$ is a field of characteristic zero.

We recall the construction of $V$ in Section 2: We have a free abelian group $\mathrm{N}_{0}=$ $\mathbb{Z} e_{1}+\mathbb{Z} e_{2}$ with the base $\left(e_{1}, e_{2}\right)$ and the cone $\boldsymbol{\sigma}=$ Cone $\left(e_{1}, e_{2}\right) \subset \mathrm{N}_{0} \otimes \mathbb{R}$ such that $V=\mathbb{T}_{\mathrm{N}}(\boldsymbol{\sigma})$ for a free abelian group $\mathrm{N}=\mathrm{N}_{0}+\mathbb{Z} v$ defined by the vector

$$
v=\frac{1}{d n^{2}}\left(e_{1}+(d n a-1) e_{2}\right) \text {. }
$$

Let $\mathrm{M}$ and $\mathrm{M}_{0}$ be the dual abelian groups of $\mathrm{N}$ and $\mathrm{N}_{0}$, respectively. Then, $R_{0}=\Lambda\left[\boldsymbol{\sigma}^{\vee} \cap \mathrm{M}_{0}\right]$ is a polynomial $\Lambda$-algebra generated by two-variables $\mathrm{x}_{1}, \mathrm{x}_{2}$ which correspond to the dual basis of $\left(e_{1}, e_{2}\right)$. In particular, $V_{0}=\mathbb{T}_{\mathrm{N}_{0}}(\boldsymbol{\sigma}) \simeq \mathbb{A}_{\Lambda}^{2}$. The affine coordinate ring $R:=\mathrm{H}^{0}\left(V, \mathcal{O}_{V}\right)=\Lambda\left[\boldsymbol{\sigma}^{\vee} \cap \mathrm{M}\right]$ is a $\Lambda$-subalgebra of $\Lambda\left[\mathrm{x}_{1}, \mathrm{x}_{2}\right]$ generated by the monomials $\mathrm{x}_{1}^{k_{1}} \mathrm{x}_{2}^{k_{2}}$ satisfying $k_{1}+(d n a-1) k_{2} \equiv 0 \bmod d n^{2}$.

In this situation, we define a subgroup $\mathrm{N}_{1}$ of $\mathrm{N}$ by

$$
\mathrm{N}_{1}:=\mathrm{N}_{0}+\mathbb{Z} n v=\mathrm{N}_{0}+\mathbb{Z} \frac{1}{d n}\left(e_{1}+(d n-1) e_{2}\right) .
$$

Then, we have a toric $\Lambda$-scheme $V_{1}=\mathbb{T}_{\mathrm{N}_{1}}(\boldsymbol{\sigma})$, and finite surjective morphisms $\tau_{0}: V_{0} \rightarrow V_{1}$ and $\tau: V_{1} \rightarrow V$ associated with the inclusions $\mathrm{N}_{0} \subset \mathrm{N}_{1}$ and $\mathrm{N}_{1} \subset \mathrm{N}$. The affine coordinate ring $R_{1}:=\mathrm{H}^{0}\left(V_{1}, \mathcal{O}_{V_{1}}\right)=\Lambda\left[\boldsymbol{\sigma}^{\vee} \cap \mathrm{M}_{1}\right]$, where $\mathrm{M}_{1}$ is the dual abelian group of $\mathrm{N}_{1}$, is a $\Lambda$-subalgebra of $\Lambda\left[\mathrm{x}_{1}, \mathrm{x}_{2}\right]$ generated by three monomials $\mathrm{x}_{1}^{d n}, \mathrm{x}_{2}^{d n}, \mathrm{x}_{1} \mathrm{x}_{2}$. Thus,

$$
R_{1} \simeq \Lambda\left[\mathrm{u}_{1}, \mathrm{u}_{2}, \mathrm{z}\right] /\left(\mathrm{u}_{1} \mathrm{u}_{2}-\mathrm{z}^{d n}\right)
$$

for three variables $\mathrm{u}_{1}, \mathrm{u}_{2}, \mathrm{z}$, where the isomorphism above is defined by $\mathrm{u}_{1}=\mathrm{x}_{1}^{d n}, \mathrm{u}_{2}=\mathrm{x}_{2}^{d n}$, and $\mathbf{z}=\mathrm{x}_{1} \mathrm{x}_{2}$. Since $n$ is the index of $\mathrm{N} / \mathrm{N}_{1}$, the group subscheme $\boldsymbol{\mu}_{n}=\operatorname{Ker}\left(\mathbb{T}_{\mathrm{N}_{1}} \rightarrow \mathbb{T}_{\mathrm{N}}\right)$ of $\mathbb{T}_{\mathrm{N}_{1}}$ acts on $V_{1}$ and its quotient scheme is just $V$. Here, the action of $\boldsymbol{\mu}_{n}$ on $V_{1}=\operatorname{Spec} R_{1}$ is given by

$$
\left(\mathrm{u}_{1}, \mathrm{u}_{2}, \mathrm{z}\right) \mapsto\left(\mathrm{u}_{1} \otimes \mathrm{t}, \mathrm{u}_{2} \otimes \mathrm{t}^{-1}, \mathrm{z} \otimes \mathrm{t}^{a}\right),
$$

where $\boldsymbol{\mu}_{n}$ is regarded as $\operatorname{Spec} \Lambda\left[\mathrm{t}, \mathrm{t}^{-1}\right] /\left(\mathrm{t}^{n}-1\right)$. The action of $\boldsymbol{\mu}_{n}$ on $V_{1}$ is induced from that on $\operatorname{Spec} \Lambda\left[\mathrm{u}_{1}, \mathrm{u}_{2}, \mathbf{z}\right]$ given by (III-3). Then, the $\boldsymbol{\mu}_{n}$-invariant part $R^{\sim}$ of $\Lambda\left[\mathrm{u}_{1}, \mathrm{u}_{2}, \mathbf{z}\right]$ is generated by monomials $u_{1}^{m_{1}} u_{2}^{m_{2}} z^{m_{3}}$ such that $m_{i} \geq 0$ for all $1 \leq i \leq 3$ and $m_{1}-m_{2}+$ $a m_{3} \equiv 0 \bmod n$. We see that $\operatorname{Spec} R^{\sim}$ is isomorphic to $\mathbb{T}_{\mathrm{N} \sim}\left(\boldsymbol{\sigma}^{\sim}\right)$ for the affine toric $\Lambda$ scheme $\mathbb{T}_{\mathrm{N}^{\sim}}\left(\boldsymbol{\sigma}^{\sim}\right)$ of relative dimension three defined as follows: Let $\mathrm{N}_{1}^{\sim}$ be a free abelian group $\bigoplus_{i=1}^{3} \mathbb{Z} e_{i}^{\sim}$ of rank three and let $\boldsymbol{\sigma}^{\sim}$ be the cone $\sum_{i=1}^{3} \mathbb{R}_{\geq 0} e_{i}^{\sim}$. The abelian group $\mathrm{N}^{\sim}$ is a subgroup of $\mathbf{N}_{1}^{\sim} \otimes \mathbb{Q}$ defined by

$$
\mathbf{N}^{\sim}:=\mathbf{N}_{1}^{\sim}+\mathbb{Z} \frac{1}{n}\left(e_{1}^{\sim}-e_{2}^{\sim}+a e_{3}^{\sim}\right) .
$$


Then, $V=\operatorname{Spec} R$ is a hypersurface (or a Cartier divisor) of $V^{\sim}:=\operatorname{Spec} R^{\sim}$ defined by the principal ideal $\left(\mathrm{u}_{1} \mathrm{u}_{2}-\mathrm{z}^{d n}\right)$.

Lemma 3.9. The smallest integer $r$ such that $r K_{V}$ (resp. $\left.r K_{V \sim}\right)$ is Cartier, is n. Thus, $n$ is equal to the Gorenstein index of $V\left(\right.$ resp. $\left.V^{\sim}\right)$.

Proof. Let $\left(k_{1}, k_{2}, k_{3}\right)$ be an integral vector such that $k_{1}-k_{2}+a k_{3} \equiv 0 \bmod n$. Then, the principal divisor associated to the monomial $\mathrm{u}_{1}^{k_{1}} \mathrm{u}_{2}^{k_{2}} \mathrm{z}^{k_{3}}$ on $V$ is expressed as

$$
\operatorname{div}\left(\mathrm{u}_{1}^{k_{1}} \mathrm{u}_{2}^{k_{2}} \mathrm{z}^{k_{3}}\right)_{V}=\left(k_{1} d n+k_{3}\right) D_{1}+\left(k_{2} d n+k_{3}\right) D_{2}
$$

by (II-6) in Remark 2.4.1. Note that $D:=D_{1}+D_{2} \sim-K_{V}$. Hence, $n$ is the Gorenstein index of $V$. In fact, $n D=\operatorname{div}\left(\mathbf{z}^{n}\right)$ is Cartier, and if $j D$ is an Cartier divisor for an integer $j$, then $j=k_{1} d n+k_{3}=k_{2} d n+k_{3}$ for some integral vector $\left(k_{1}, k_{2}, k_{3}\right)$ above; hence $k_{1}=k_{2}$ and $j \equiv k_{3} \equiv 0 \bmod n$.

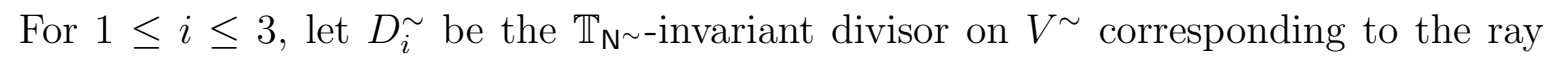
$\mathbb{R}_{\geq 0} e_{i}^{\sim}$. Since $\operatorname{gcd}(n, a)=1$, we see that

$$
\mathbb{R}_{\geq 0} e_{i}^{\sim} \cap \mathbf{N}^{\sim}=\mathbb{Z}_{\geq 0} e_{i}^{\sim}
$$

for $1 \leq i \leq 3$. Then, we have the following equality similar to $(\underline{\underline{I I}-6})$ :

$$
\operatorname{div}\left(\mathrm{u}_{1}^{k_{1}} \mathrm{u}_{2}^{k_{2}} \mathrm{z}^{k_{3}}\right)_{V^{\sim}}=k_{1} D_{1}^{\sim}+k_{2} D_{2}^{\sim}+k_{3} D_{3}^{\sim} .
$$

Here, we know also $D^{\sim}:=\sum_{i=1}^{3} D_{i}^{\sim} \sim-K_{V^{\sim}}$ and that $n D_{i}^{\sim}$ is Cartier for all $i$. Suppose that $j D^{\sim}$ is Cartier for an integer $j$. Then, $j=k_{1}=k_{2}=k_{3}$ for an integral vector $\left(k_{1}, k_{2}, k_{3}\right)$ such that $k_{1}-k_{2}+a k_{3} \equiv 0 \bmod n$; hence $j \equiv 0 \bmod n$. Therefore, the Gorenstein index of $V^{\sim}$ is also $n$.

Remark 3.9.1. $V$ and $V^{\sim}$ are $\mathbb{Q}$-factorial, since the cones $\boldsymbol{\sigma}$ and $\boldsymbol{\sigma}^{\sim}$ are simplicial.

Remark 3.9.2. It is well-known in characteristic zero that the singularity on $V^{\sim}$ is a cyclic quotient terminal singularity of type $\frac{1}{n}(1,-1, a)$ (cf. [45, §3], [46, (4.13)], [47, (5.1), $(5.2)])$. Even in the positive characteristic case, the singularity is "terminal" in the sense that there is a resolution $\mu^{\sim}: M^{\sim} \rightarrow V^{\sim}$ of singularity such that

$$
K_{M^{\sim}}=\mu^{\sim *}\left(K_{V^{\sim}}\right)+\sum a_{i} E_{i}^{\sim}
$$

for $\mu^{\sim}$-exceptional prime divisors $E_{i}^{\sim}$ and positive rational numbers $a_{i}$. In fact, a toric resolution of $V^{\sim}$ is taken independently of the characteristic, and the discrepancy $a_{i}$ is also independent of the characteristic.

Remark 3.9.3. If $X$ is a $\mathbb{Q}$-Gorenstein normal algebraic $\Lambda$-scheme and if $Y$ is a normal Cartier divisor of $X$, then $\left.r\left(K_{X}+Y\right)\right|_{Y} \sim r K_{Y}$ for the Gorenstein index $r$ of $X$. Indeed, 
the left hand side is Cartier and is linearly equivalent to the right hand side on the non-singular locus of $Y$. In particular, $Y$ is also $\mathbb{Q}$-Gorenstein and $r$ is divisible by the $\mathbb{Q}$ Gorenstein index of $Y$. Applying this to Lemma 3.9, we see that the restriction $\left.n K_{V^{\sim}}\right|_{V}$ is linearly equivalent to $n K_{V}$.

Proof of Theorem 3.8. We consider an algebra

$$
R_{1}^{\sharp}:=\Lambda\left[\mathrm{u}_{1}, \mathrm{u}_{2}, \mathrm{z}, \mathrm{s}\right] /\left(\mathrm{z}^{d n}-\mathrm{u}_{1} \mathrm{u}_{2}-\mathrm{s}\left(\mathrm{z}^{n}+1\right)\right)
$$

over the polynomial ring $\Lambda[\mathbf{s}]$ of one variable. This is flat over $\Lambda[\mathbf{s}]$, since it is CohenMacaulay and every fiber of $\operatorname{Spec} R_{1}^{\sharp} \rightarrow \operatorname{Spec} \Lambda[\mathrm{s}]$ is equidimensional. We consider the $\boldsymbol{\mu}_{n}$-action on Spec $R_{1}^{\sharp}$ given by

$$
\left(\mathrm{u}_{1}, \mathrm{u}_{2}, \mathrm{z}, \mathrm{s}\right) \mapsto\left(\mathrm{tu}_{1}, \mathrm{t}^{-1} \mathrm{u}_{2}, \mathrm{t}^{a} \mathrm{z}, \mathrm{s}\right)
$$

where $\boldsymbol{\mu}_{n}=\operatorname{Spec} \Lambda\left[\mathrm{t}, \mathrm{t}^{-1}\right] /\left(\mathrm{t}^{n}-1\right)$. Let $R^{\sharp}$ be the $\boldsymbol{\mu}_{n}$-invariant subring, which is the $\Lambda[\mathbf{s}]$-submodule generated by monomials $u_{1}^{k_{1}} u_{2}^{k_{2}} z^{k_{3}}$ with $k_{1}-k_{2}+a k_{3} \equiv 0 \bmod n$. We set $V^{\sharp}:=\operatorname{Spec} R^{\sharp}, S:=\operatorname{Spec} \Lambda[\mathrm{s}]=\mathbb{A}_{\Lambda}^{1}$, and let $\sigma: \operatorname{Spec} \Lambda \rightarrow S$ be the section defined by $\Lambda[\mathbf{s}] \rightarrow \Lambda[\mathbf{s}] /(\mathbf{s}) \simeq \Lambda$. Then, $V^{\sharp} \times_{S, \sigma}$ Spec $\Lambda \simeq V$. Since $R^{\sharp}$ is the $\boldsymbol{\mu}_{n}$-invariant ring of $R_{1}^{\sharp}$, which is a direct summand of $R_{1}^{\sharp}$, we see that $V^{\sharp}$ is normal and $V^{\sharp} \rightarrow S$ is flat. On the other hand, the affine scheme $V^{\sharp}$ is isomorphic to the hypersurface of $V^{\sim} \times_{\operatorname{Spec} \Lambda} S$ defined by $\mathbf{z}^{d n}-\mathrm{u}_{1} \mathrm{u}_{2}-s\left(\mathbf{z}^{n}+1\right)=0$. By Lemma 3.9, $V^{\sim} \times_{\operatorname{Spec} \Lambda} S$ is $\mathbb{Q}$-Gorenstein with index $n$. Hence, $V^{\sharp}$ is also $\mathbb{Q}$-Gorenstein with index $r$ dividing $n$, since $V^{\sharp}$ is normal (cf. Remark 3.9.3). Here, $r=n$ by Lemma [3.9, since $V$ is also a hypersurface of $V^{\sharp}$. Consequently, we have $\left.\mathcal{O}_{V^{\sharp}}\left(n K_{V^{\sharp}}\right)\right|_{V} \sim \mathcal{O}_{V}\left(n K_{V}\right)$ (cf. Remark 3.9.3).

We shall study the singularity of fibers of $V^{\sharp} \rightarrow S$ outside the section $\sigma(\operatorname{Spec} \Lambda)$ defined by $\mathrm{s}=0$. Let $R^{\sharp}\langle 1\rangle$ be the affine coordinate ring of the affine open subset $\left\{\mathrm{u}_{1} \neq 0, \mathrm{~s} \neq 0\right\}$ of Spec $R^{\sharp}$. Then, $R^{\sharp}\langle 1\rangle$ is isomorphic to

$$
\Lambda\left[\mathrm{y}_{1}^{ \pm 1}, \mathrm{y}_{2}, \mathrm{y}_{3}, \mathrm{~s}^{ \pm 1}\right] /\left(\mathrm{y}_{3}^{d n} \mathrm{y}_{1}^{a d}-\mathrm{y}_{2}-\mathrm{s}\left(\mathrm{y}_{3}^{n} \mathrm{y}_{1}^{a}+1\right)\right) \simeq \Lambda\left[\mathrm{y}_{1}^{ \pm 1}, \mathrm{y}_{3}, \mathrm{~s}^{ \pm 1}\right]
$$

by the correspondence $\left(\mathrm{y}_{1}, \mathrm{y}_{2}, \mathrm{y}_{3}\right)=\left(\mathrm{u}_{1}^{n}, \mathrm{u}_{1} \mathrm{u}_{2}, \mathrm{zu}_{1}^{-a}\right)$. Thus, $R^{\sharp}\langle 1\rangle$ is smooth over $\Lambda\left[\mathrm{s}^{ \pm 1}\right]$. Similarly, the affine coordinate $\operatorname{ring} R^{\sharp}\langle 2\rangle$ of $\left\{\mathrm{u}_{2} \neq 0, \mathbf{s} \neq 0\right\}$ is smooth over $\Lambda\left[\mathbf{s}^{ \pm 1}\right]$, since it is isomorphic to

$$
\Lambda\left[\mathrm{y}_{1}, \mathrm{y}_{2}^{ \pm 1}, \mathrm{y}_{3}, \mathrm{~s}^{ \pm 1}\right] /\left(\mathrm{y}_{3}^{d n} \mathrm{y}_{2}^{-a d}-\mathrm{y}_{1}-\mathrm{s}\left(\mathrm{y}_{3}^{n} \mathrm{y}_{2}^{-a}+1\right)\right) \simeq \Lambda\left[\mathrm{y}_{2}^{ \pm 1}, \mathrm{y}_{3}, \mathrm{~s}^{ \pm 1}\right]
$$

by the correspondence $\left(\mathrm{y}_{1}, \mathrm{y}_{2}, \mathrm{y}_{3}\right)=\left(\mathrm{u}_{1} \mathrm{u}_{2}, \mathrm{u}_{2}^{n}, \mathrm{zu}_{2}^{a}\right)$.

The affine coordinate ring $R^{\sharp}\langle 3\rangle$ of $\{\mathbf{z} \neq 0, \mathbf{s} \neq 0\}$ is isomorphic to

$$
\Lambda\left[\mathrm{y}_{1}, \mathrm{y}_{2}, \mathrm{y}_{3}^{ \pm 1}, \mathrm{~s}^{ \pm 1}\right] /\left(\mathrm{y}_{3}^{d}-\mathrm{y}_{1} \mathrm{y}_{2}-\mathrm{s}\left(\mathrm{y}_{3}+1\right)\right)
$$

by the correspondence $\left(\mathrm{y}_{1}, \mathrm{y}_{2}, \mathrm{y}_{3}\right)=\left(\mathrm{u}_{1} \mathrm{z}^{-a^{\prime}}, \mathrm{u}_{2} \mathbf{z}^{a^{\prime}}, \mathrm{z}^{n}\right)$, where $0<a^{\prime}<n$ with $a a^{\prime} \equiv 1$ $\bmod n$. Let $p$ be the characteristic of the residue field of $\Lambda$. If $p \mid d$ or $p \mid d-1$, then $R^{\sharp}\langle 3\rangle$ 
is smooth over $\Lambda\left[\mathbf{s}^{ \pm 1}\right]$ by the Jacobian criterion. If $p \nmid d$ and $p \nmid d-1$, then $R^{\sharp}\langle 3\rangle$ is smooth over $\Lambda\left[\mathbf{s}^{ \pm 1},(\mathbf{s}-c)^{-1}\right]$ for $c=d^{d} /(d-1)^{d-1}$ by the Jacobian criterion. Thus, the open subset $T:=\operatorname{Spec} \Lambda\left[\mathbf{s},(\mathrm{s}-c)^{-1}\right]$ of $S=\mathbb{A}_{\Lambda}^{1}, \sigma: \operatorname{Spec} \Lambda \rightarrow T \subset S$, and $\mathcal{V}:=V^{\sharp} \times_{S} T \rightarrow T$ satisfy all the conditions of Theorem 3.8 .

\section{REVIEW OF DEFORMATION THEORY}

We review the deformation theory of $\mathbb{k}$-schemes for the fixed algebraically closed field $\mathbb{k}$.

Definition 4.1. Let $X$ be a $\mathbb{k}$-scheme. Let $T$ be a scheme and let $o$ be a $\mathbb{k}$-rational point of $T$, which is just a morphism $o:$ Spec $\mathbb{k} \rightarrow T$. A deformation of $X$ over $T$ with the reference point $o$ is a pair $(Y / T, \iota)$ of a flat morphism $Y \rightarrow T$ of schemes and an isomorphism $\iota: Y \times_{T, o}$ Spec $\mathbb{k} \simeq X$ over Spec $\mathbb{k}$.

In this section, we fix a Noetherian local ring $\Lambda$ which is either $\mathbb{k}$ or a complete discrete valuation ring with residue field $\mathbb{k}$. For example, the ring of Witt vectors of $\mathbb{k}$ is a candidate of $\Lambda$.

We recall some important notions mainly from Schlessinger's article [49]. Let $\mathcal{C}_{\Lambda}$ be the category of Artinian local $\Lambda$-algebras with residue field $\mathbb{k}$ and let $\widehat{\mathcal{C}_{\Lambda}}$ be the category of complete Noetherian local $\Lambda$-algebras $\mathfrak{R}=\left(\mathfrak{R}, \mathfrak{m}_{\mathfrak{R}}\right)$ such that $\mathfrak{R} / \mathfrak{m}_{\mathfrak{R}}^{n} \in \mathcal{C}_{\Lambda}$ for all $n$. An object $\mathfrak{R}$ of $\widehat{\mathcal{C}_{\Lambda}}$ defines a functor $h_{\Re}: \mathcal{C}_{\Lambda} \rightarrow$ (Sets) to the category of sets by $h_{\Re}(A)=\operatorname{Hom}_{\widehat{\mathcal{C}}_{\Lambda}}(\mathfrak{R}, A)$. For two functors $F, G: \mathcal{C}_{\Lambda} \rightarrow$ (Sets), a morphism $\phi: F \rightarrow G$ means a natural transformation of functors. The morphism $\phi: F \rightarrow G$ is called smooth (in the sense of Schlessinger [49, (2.2)]) if the natural map $F(B) \rightarrow F(A) \times_{G(A)} G(B)$ is surjective for any surjection $B \rightarrow A$ in $\mathcal{C}_{\Lambda}$.

The deformation functor $\operatorname{Def}_{X}: \mathcal{C}_{\Lambda} \rightarrow$ (Sets) of a $\mathbb{k}$-scheme $X$ is defined as follows. An infinitesimal deformation of $X$ to an algebra $A$ of $\mathcal{C}_{\Lambda}$ is a deformation $\left(X_{A}, \iota\right)$ of $X$ over $\operatorname{Spec} A$ with $\mathfrak{m}_{A}$ as a $\mathbb{k}$-rational reference point . Here, $X_{A}$ is a flat $A$-scheme and $\iota$ is an isomorphism $X_{A} \times_{\operatorname{Spec} A} \operatorname{Spec} \mathbb{k} \simeq X$. Another deformation $\left(X_{A}^{\prime}, \iota^{\prime}\right)$ of $X$ is isomorphic to $\left(X_{A}, \iota\right)$ if there is a morphism $\phi: X_{A} \rightarrow X_{A}^{\prime}$ over Spec $A$ such that $\iota^{\prime}=\phi \circ \iota$. Note that this morphism $\phi$ is indeed an isomorphism over Spec $A$, since $X_{A}$ and $X_{A}^{\prime}$ are both homeomorphic to $X$ and since $\phi$ induces the identity on $X$. We define $\operatorname{Def}_{X}(A)$ to be the set of isomorphism classes of deformations of $X$ to $A$. Then, $\operatorname{Def}_{X}$ gives rise to a functor $\mathcal{C}_{\Lambda} \rightarrow$ (Sets), which is called the deformation functor of $X$. When $X$ is an affine scheme Spec $R$, we write $\operatorname{Def}_{R}$ for $\operatorname{Def}_{X}$. 
Definition 4.2 (Pro-couple, formal deformation, and hull). Let $\mathfrak{R}$ be an object of $\widehat{\mathcal{C}}_{\Lambda}$ and set $\mathfrak{R}_{n}:=\mathfrak{R} / \mathfrak{m}_{\mathfrak{R}}^{n+1}$ for $n \geq 0$. Let $\xi$ be an element of

$$
\widehat{\operatorname{Def}_{X}}(\mathfrak{R}):=\lim _{n} \operatorname{Def}_{X}\left(\mathfrak{R}_{n}\right) \text {. }
$$

Note that to give an element of $\widehat{\operatorname{Def}_{X}}(\mathfrak{R})$ is equivalent to giving a morphism $h_{\Re} \rightarrow \operatorname{Def}_{X}$ of functors. In [49], the pair $(\Re, \xi)$ is called a pro-couple. For each $n$, the element $\xi$ defines a flat $\mathfrak{R}_{n}$-scheme $X_{n}$ with an isomorphism $\iota_{n}: X_{n} \times_{\text {Spec } \Re_{n}}$ Spec $\mathbb{k} \simeq X$. Moreover, $\left(X_{n}, \iota_{n}\right)$ form an inductive system. Thus, we have a formal scheme $\mathfrak{X}=\mathfrak{X}_{\xi}:=\underline{\lim } X_{n}$ flat over the affine formal scheme Spf $\mathfrak{R}$ with an isomorphism $\iota: \mathfrak{X} \times{ }_{\operatorname{Spf} \Re} \operatorname{Spec} \mathbb{k} \simeq X$ (cf. [EGA, I, Proposition (10.6.3)]). The pair $(\mathfrak{X} / \operatorname{Spf} \mathfrak{R}, \iota)$ or the system $\left(X_{n}, \iota_{n}\right)_{n \geq 0}$ is called a formal deformation of $X$ over $\mathfrak{R}$ (or over Spf $\mathfrak{R}$ ).

Remark 4.2.1. In the definition above, if $X$ is an algebraic $\mathbb{k}$-scheme, i.e., a $\mathbb{k}$-scheme of finite type, then $\mathfrak{X}$ is Noetherian and $\mathfrak{X} \rightarrow \operatorname{Spf} \mathfrak{R}$ is a morphism of finite type by [EGA, I, Corollaire (10.6.4), Proposition (10.13.1), Définition (10.13.3)]. If $X$ is proper over $\mathbb{k}$, then $\mathfrak{X} \rightarrow$ Spf $\mathfrak{R}$ is proper (cf. [EGA, III, (3.4.1)]).

Definition 4.3. Let $(\mathfrak{R}, \xi)$ be a pro-couple of $\operatorname{Def}_{X}$ and let $\mathfrak{X} \rightarrow$ Spf $\mathfrak{R}$ be the formal deformation of $X$ associated to $(\Re, \xi)$.

(1) The pro-couple (or the formal deformation) is said to be effective if there is a flat $\mathfrak{R}$-scheme $\mathcal{X}$ such that $\mathcal{X}$ is a deformation of $X$ over Spec $\mathfrak{R}$ and that $(\mathfrak{R}, \xi)$ is induced from the inductive system $\mathcal{X}_{n}=\mathcal{X} \times_{\text {Spec } \Re}$ Spec $\Re_{n}$; equivalently the $\mathfrak{m}_{\mathfrak{R}}$-adic completion of $\mathcal{X}$ is isomorphic to $\mathfrak{X}$ as a formal scheme over Spf $\mathfrak{R}$.

(2) The pro-couple (or the formal deformation) is said to be algebraizable if there is a deformation $Y \rightarrow T$ of $X$ with a $\mathbb{k}$-rational reference point $o \in T$ such that

- $T$ is a scheme of finite type over $\Lambda$,

- the completion of the local ring $\mathcal{O}_{T, o}$ is isomorphic to $\mathfrak{R}$, and

- the formal completion of $Y$ along the fiber $X$ is isomorphic to $\mathfrak{X}$ over Spf $\mathfrak{R}$.

(3) The pro-couple $(\mathfrak{R}, \xi)$ is called a pro-representable hull (or a hull, for short) of $\operatorname{Def}_{X}$ if the morphism $h_{\Re} \rightarrow \operatorname{Def}_{X}$ corresponding to $\xi$ is smooth and induces the bijection

$$
\boldsymbol{t}\left(h_{\mathfrak{R}}\right):=h_{\mathfrak{R}}\left(\mathbb{k}[\varepsilon] /\left(\varepsilon^{2}\right)\right) \rightarrow \boldsymbol{t}\left(\operatorname{Def}_{X}\right):=\operatorname{Def}_{X}\left(\mathbb{k}[\varepsilon] /\left(\varepsilon^{2}\right)\right)
$$

between the tangent spaces (cf. [49, Definition 2.7]).

Remark 4.3.1. The hull is unique up to non-canonical isomorphism (cf. [49, Proposition 2.9]). The existence of hull of $\operatorname{Def}_{X}$ is known in the cases when $X$ is proper over $\mathbb{k}$ and when $X$ is affine with only isolated singularities (cf. [49, Proposition 3.10]). 
Remark 4.3.2. Let $X$ be a projective $\mathbb{k}$-scheme and let $\mathfrak{X} \rightarrow$ Spf $\mathfrak{R}$ be the formal deformation associated with a pro-representable hull $(\mathfrak{R}, \xi)$ of $\operatorname{Def}_{X}$. If $\mathfrak{X}$ admits a relatively ample invertible sheaf over Spf $\mathfrak{R}$, then this is effective by a projective morphism $\mathcal{X} \rightarrow \operatorname{Spec} \mathfrak{R}$, by an application [EGA, III, Théorème (5.4.5)] of Grothendieck's existence theorem, i.e., $\mathfrak{X}$ is the $\mathfrak{m}_{\mathfrak{R}}$-adic completion of $\mathcal{X}$. Moreover, this is algebraizable by Artin's result [3, Theorem 1.6].

Remark 4.3.3. Let $X$ be an equidimensional affine algebraic $\mathbb{k}$-scheme with only isolated singularities. Then, the pro-representable hull of $\operatorname{Def}_{X}$ is effective by [14, Chapitre IV, Théorème 7] and is algebraizable by [3, Theorem 1.6].

Lemma 4.4. If $X$ is an affine algebraic $\mathbb{k}$-scheme with a unique singular point $x$ and if $\left(X^{\prime}, x^{\prime}\right)$ is an étale neighborhood of $(X, x)$, then there is a smooth morphism Def $_{X} \rightarrow$ $\operatorname{Def}_{\mathcal{O}_{X^{\prime}, x^{\prime}}}$ of functors on $\mathcal{C}_{\Lambda}$ inducing an isomorphism between the tangent spaces.

Proof. We have a morphism $\operatorname{Def}_{X} \rightarrow \operatorname{Def}_{\mathcal{O}_{X^{\prime}, x^{\prime}}}$ by [EGA, IV, Théorème (18.1.2)]. The smoothness and the isomorphism between the tangent spaces are derived from [48, Theorem 4.10.(b)].

Remark 4.4.1. If $(X, x)$ is non-singular, then $\operatorname{Def}_{\mathcal{O}_{X, x}}(A)$ consists of one element for any $A \in \mathcal{C}_{\Lambda}$. In other words, the canonical morphism $\operatorname{Def}_{R} \rightarrow h_{\Lambda}$ of functors is an isomorphism. This follows for example from [EGA, IV, Proposition (18.1.1)] and the formal smoothness (cf. [EGA, IV, Définition (17.1.1)]) of $\operatorname{Spec} \mathcal{O}_{X, x} \rightarrow \operatorname{Spec} \mathbb{k}$.

Lemma 4.4.2. Let $(X, x)$ and $\left(X^{\prime}, x^{\prime}\right)$ be as in Lemma 4.4. Let $Y \rightarrow T$ be a deformation of $\operatorname{Spec} \mathcal{O}_{X, x}$ over a scheme $T$ which is with a $\mathbb{k}$-rational reference point $o \in T$ such that $Y$ is an affine scheme of a local ring. Then, there exist a deformation $Y^{\prime} \rightarrow T$ of $\operatorname{Spec} \mathcal{O}_{X^{\prime}, x^{\prime}}$ over $T$ with reference point $o$ and a formally étale morphism $Y^{\prime} \rightarrow Y$ over $T$ inducing $\operatorname{Spec} \mathcal{O}_{X^{\prime}, x^{\prime}} \rightarrow \operatorname{Spec} \mathcal{O}_{X, x}$ as a morphism between the fibers over o.

Proof. This follows from [EGA, IV, Proposition (18.1.1)], since $\mathcal{O}_{X, x}$ and $\mathcal{O}_{X^{\prime}, x^{\prime}}$ are essentially of finite type over $\mathbb{k}$.

Definition 4.5. Let $X$ be an algebraic $\mathbb{k}$-scheme and $P$ a $\mathbb{k}$-rational point. We write $\operatorname{Def}_{(X, P)}:=\operatorname{Def}_{\mathcal{O}_{X, P}}$. When $X$ is non-singular outside a finite set (i.e., has only isolated singularities), we define a functor $\operatorname{Def}_{X}^{(\text {loc })}$ on $\mathcal{C}_{\Lambda}$ by

$$
\operatorname{Def}_{X}^{(\text {loc })}(A):=\prod_{P \in \operatorname{Sing} X} \operatorname{Def}_{(X, P)}(A)
$$

for $A \in \mathcal{C}_{\Lambda}$, where $\operatorname{Sing} X$ stands for the singular locus. 
Remark 4.5.1. There is a natural morphism $\operatorname{Def}_{X} \rightarrow \operatorname{Def}_{(X, P)}$ of functors for any $\mathbb{k}$ rational point $P \in X$ which sends $\left(X_{A}, \iota\right) \in \operatorname{Def}_{X}(A)$ to the pair consisting of the local $A$-algebra $\mathcal{O}_{X_{A}, \iota(P)}$ and the isomorphism $\mathcal{O}_{X, P} \simeq \mathcal{O}_{X_{A}, \iota(P)} \otimes_{A} \mathbb{k}$ induced by $\iota$. As a consequence, we have a natural morphism $\operatorname{Def}_{X} \rightarrow \operatorname{Def}_{X}^{(\text {loc })}$ of functors when $X$ has only isolated singularities.

We shall give a proof of the following well-known:

Theorem 4.6 ([55, Proposition 6.4]). Let $X$ be an algebraic $\mathbb{k}$-scheme with only isolated singularities. Assume that $\mathrm{H}^{2}\left(X, \Theta_{X / \mathbb{k}}\right)=0$, where $\Theta_{X / \mathbb{k}}$ denotes the tangent sheaf $\mathcal{H}_{\text {om }} \mathcal{O}_{X}\left(\Omega_{X / \mathbb{k}}^{1}, \mathcal{O}_{X}\right)$. Then, the morphism $\operatorname{Def}_{X} \rightarrow \operatorname{Def}_{X}^{(\text {loc })}$ of functors is smooth.

Proof. Let $B \rightarrow A$ be a surjection in $\mathcal{C}_{\Lambda}$ with the kernel $I$ satisfying $I \mathfrak{m}_{B}=0$. It suffices to prove that

$$
\operatorname{Def}_{X}(B) \rightarrow \operatorname{Def}_{X}(A) \times \operatorname{Def}_{X}^{(\text {loc })}(A) \operatorname{Def}_{X}^{(\text {loc })}(B)
$$

is surjective. An element of the right-hand side of (IV-1) consists of

- a flat $A$-scheme $X_{A}$ with an isomorphism $\iota_{A}: X_{A} \times_{\operatorname{Spec} A} \operatorname{Spec} \mathbb{k} \simeq X$, and

- flat $B$-algebras $S_{B}^{(P)}$ for any $P \in \operatorname{Sing} X$ with isomorphisms $\iota_{B}^{(P)}: S_{B}^{(P)} \otimes_{B} \mathbb{k} \simeq \mathcal{O}_{X, P}$, where, for any $P \in \operatorname{Sing} X$, we can find an isomorphism $\Psi_{P}: \mathcal{O}_{X_{A}, P} \stackrel{\simeq}{\rightarrow} S_{B}^{(P)} \otimes_{B} A$ such that the composite

$$
\mathcal{O}_{X_{A}, P} \stackrel{\Psi_{P}}{\longrightarrow} S_{B}^{(P)} \otimes_{B} A \rightarrow S_{B}^{(P)} \otimes_{B} \mathbb{k} \stackrel{\iota_{B}^{(P)}}{\longrightarrow} \mathcal{O}_{X, P}
$$

is the homomorphism induced by $\iota_{A}$.

We apply the obstruction theory of infinitesimal deformations using the cotangent complexes (cf. [20, 34]): Let $\mathbb{L}_{Z / Y}$ be the cotangent complex (as an object of the derived category $\left.D^{-}(\operatorname{Coh}(Z))\right)$ for a morphism $Z \rightarrow Y$ of schemes. For a coherent sheaf $\mathcal{F}$ on $Z$, we denote the associated cohomology groups/sheaves by

$$
\begin{aligned}
& \mathrm{T}^{i}(Z / Y, \mathcal{F})=\operatorname{Ext}^{i}\left(\mathbb{L}_{Z / Y}, \mathcal{F}\right)=\mathrm{H}^{i}\left(\operatorname{RHom}_{\mathcal{O}_{Z}}\left(\mathbb{L}_{Z / Y}, \mathcal{F}\right)\right), \\
& \mathcal{T}^{i}(Z / Y, \mathcal{F})=\underline{\operatorname{Ext}^{i}}\left(\mathbb{L}_{Z / Y}, \mathcal{F}\right)=\mathcal{H}^{i}\left(\mathcal{R H} \text { om }_{\mathcal{O}_{Z}}\left(\mathbb{L}_{Z / Y}, \mathcal{F}\right)\right)
\end{aligned}
$$

for $i \geq 0$. When $Y$ is the affine scheme $\operatorname{Spec} A$, we write "/ $A$ " instead of "/ Spec $A$ ". If $Z$ is also affine in addition, then $Z$ is replaced with the coordinate ring. We recall a few properties on $\mathrm{T}^{i}(Z / Y, \mathcal{F})$ and $\mathcal{T}^{i}(Z / Y, \mathcal{F})$.

(i) $\mathrm{T}^{0}(Z / Y, \mathcal{F}) \simeq \mathcal{H o m}_{\mathcal{O}_{Z}}\left(\Omega_{Z / Y}^{1}, \mathcal{F}\right)$ (cf. [20, Chapitre II, (1.2.7.4) and Corollaire 1.2.4.3]).

(ii) If $Z \rightarrow Y$ is smooth, then $\mathrm{T}^{i}(Z / Y, \mathcal{F})=\mathcal{T}^{i}(Z / Y, \mathcal{F})=0$ for all $i>0$ (cf. [20, Chapitre III, Proposition 3.1.2]). 
(iii) If $Z \rightarrow Y$ is the base change of a flat morphism $Z_{1} \rightarrow Y_{1}$ by an affine morphism $Y \rightarrow Y_{1}$, then $\mathrm{T}^{i}(Z / Y, \mathcal{F}) \simeq \mathrm{T}^{i}\left(Z_{1} / Y_{1}, u_{*} \mathcal{F}\right)$ for the induced affine morphism $u: Z \rightarrow Z_{1}$ (cf. [34, 2.3.2], [20, Chapitre II, Corollaire 2.3.11]).

We want to find a $B$-scheme $X_{B}$, whose structure sheaf $\mathcal{O}_{X_{B}}$ is sitting inside a commutative diagram

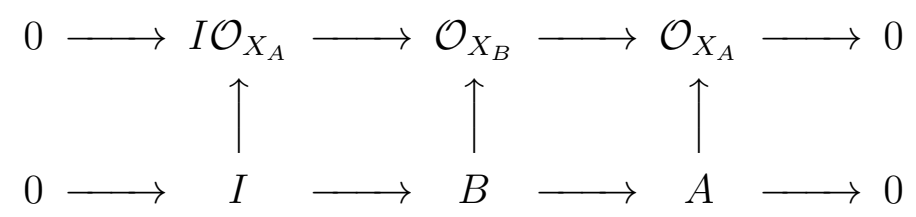

of algebra extensions, where the top exact sequence means that the ideal sheaf of $X_{A}$ in $X_{B}$ is square zero and is isomorphic to $I \mathcal{O}_{X_{A}}$ as an $\mathcal{O}_{X_{A}}$-module, and where the vertical arrows are natural homomorphisms for the $A$-scheme $X_{A}$ and the $B$-scheme $X_{B}$. Note that $I \mathcal{O}_{X_{A}} \simeq I \otimes_{\mathbb{k}} \mathcal{O}_{X}$, since $I m_{B}=0$. We write $I \mathcal{O}_{X}:=I \otimes_{\mathbb{k}} \mathcal{O}_{X}$. The obstruction class $\mathrm{ob}\left(X_{A}\right)$ for the existence of $X_{B}$ lies in the cohomology group $T^{2}\left(X_{A} / A, I \mathcal{O}_{X_{A}}\right)$ (cf. [20, Chapitre III, Théorèm 2.1.7.(i)]) which is isomorphic to $T^{2}\left(X / \mathbb{k}, I \mathcal{O}_{X}\right)$ by (iiil) above. We consider the spectral sequence

$$
E_{2}^{p, q}=\mathrm{H}^{p}\left(X, \mathcal{T}^{q}\left(X / \mathbb{k}, I \mathcal{O}_{X}\right)\right) \Rightarrow E^{p+q}=\mathrm{T}^{p+q}\left(X / \mathbb{k}, I \mathcal{O}_{X}\right) .
$$

Then, $E_{2}^{2,0}=0$ by $\mathrm{H}^{2}\left(X, \Theta_{X / \mathbb{k}}\right)=0$, since $\mathcal{T}^{0}\left(X / \mathbb{k}, I \mathcal{O}_{X}\right)=\Theta_{X / \mathbb{k}} \otimes_{\mathbb{k}} I$ by (ili). Moreover, $E_{2}^{1,1}=0$, since Sing $X$ is finite and since $\mathcal{T}^{1}\left(X / \mathbb{k}, I \mathcal{O}_{X}\right)=\mathcal{T}^{1}\left(X / \mathbb{k}, \mathcal{O}_{X}\right) \otimes_{\mathbb{k}} I$ is supported on Sing $X$ by (iii). In particular,

$$
\mathrm{T}^{1}\left(X / \mathbb{k}, I \mathcal{O}_{X}\right)=E^{1} \rightarrow E_{2}^{0,1}=\prod_{P \in \operatorname{Sing} X} \mathrm{~T}^{1}\left(\mathcal{O}_{X, P} / \mathbb{k}, I \mathcal{O}_{X, P}\right)
$$

is surjective, and

$$
\mathrm{T}^{2}\left(X / \mathbb{k}, I \mathcal{O}_{X}\right)=E^{2} \rightarrow E_{2}^{0,2}=\prod_{P \in \operatorname{Sing} X} \mathrm{~T}^{2}\left(\mathcal{O}_{X, P} / \mathbb{k}, I \mathcal{O}_{X, P}\right)
$$

is injective. The class ob $\left(X_{A}\right)$ lies in the kernel of $E^{2} \rightarrow E_{2}^{0,2}$, since $S_{A}^{(P)}$ possesses a lifting $S_{B}^{(P)}$ to $B$. Thus, $\mathrm{ob}\left(X_{A}\right)=0$. As a consequence, we have a flat $B$-scheme $X_{B}$ with an isomorphism $\iota_{B / A}: X_{B} \times_{\operatorname{Spec} B} \operatorname{Spec} A \simeq X_{A}$ over $A$. In other words, we have an element $\left(X_{B}, \iota_{B}\right)$ of $\operatorname{Def}_{X}(B)$ which is mapped to $\left(X_{A}, \iota_{A}\right) \in \operatorname{Def}_{X}(A)$. However, $\mathcal{O}_{X_{B}, P}$ may not be isomorphic to $S_{B}^{(P)}$ as a lift of $S_{A}^{(P)}$ to $B$. But, by a usual obstruction theory of deformations, the difference of two lifts lies in $T^{1}\left(S_{A}^{(P)} / A, I \mathcal{O}_{X, P}\right) \simeq T^{1}\left(\mathcal{O}_{X, P} / \mathbb{k}, I \mathcal{O}_{X, P}\right)$ (cf. [20, Chapitre III, Théorèm 2.1.7.(ii)]). Since $E^{1} \rightarrow E_{2}^{0,1}$ is surjective, we can replace the lift $X_{B}$ by another one so that $\mathcal{O}_{X_{B}, P}$ is isomorphic to $S_{B}^{(P)}$ for any $P \in \operatorname{Sing} X$. Thus, $\left(X_{B}, \iota_{B}\right)$ is mapped to the given element of the right-hand side of (IV-1). Therefore, (IV-1) is surjective. 
Finally in Section 4, we shall give the following result on algebraization related to Theorem 4.6. Roughly speaking, the result says that under suitable conditions, any given local algebraic deformations of isolated singularities extend to a global algebraic deformation.

Theorem 4.7 (algebraization). Let $X$ be a normal projective variety defined over an algebraically closed field $\mathbb{k}$ with only isolated singularities. Assume that

(i) $\mathrm{H}^{2}\left(X, \Theta_{X / \mathbb{k}}\right)=0$,

(ii) the formal deformation $\mathfrak{X} \rightarrow \operatorname{Spf} \mathfrak{R}$ associated with a pro-representable hull of Def $_{X}$ is projective, i.e., $\mathfrak{X}$ admits a relatively ample invertible sheaf over $\mathrm{Spf} \mathfrak{R}$.

Let $T$ be an algebraic $\Lambda$-scheme and let $o \in T$ be a $\mathbb{k}$-rational point. For every singular point $P \in X$, assume that we are given an affine étale neighborhood $\left(U_{(P)}, P^{\prime}\right)$ of $P$ in $X$ with a unique singular point and given a deformation $\mathcal{U}_{(P)} \rightarrow T$ of $U_{(P)}$ with reference point o. Then, there exist

- an étale neighborhood $\left(T^{\prime}, o^{\prime}\right)$ of $(T, o)$, and

- a projective morphism $Z \rightarrow T^{\prime}$ which is a deformation of $X$ with reference point $o^{\prime}$

such that, for any singular point $P,(Z, P)$ and $\left(\mathcal{U}_{(P)}, P^{\prime}\right)$ have a common étale neighborhood, i.e., the formal completions of the local rings $\mathcal{O}_{Z, P}$ and $\mathcal{O}_{\mathcal{U}_{(P)}, P^{\prime}}$ are isomorphic to each other ([2, Corollary (2.6)]).

Proof. By Remark 4.3.3 and by Lemma 4.4, for any point $P \in \operatorname{Sing} X$, there exists an algebraic deformation $W_{(P)} \rightarrow T_{(P)}$ of an affine open neighborhood of $P$ over an algebraic $\Lambda$-scheme $T_{(P)}$ such that the formal completion of the fiber induces a hull of the deformation functor $\operatorname{Def}_{(X, P)}$. By replacing $(T, o)$ with an étale neighborhood $\left(T^{\prime}, o^{\prime}\right)$, we have a morphism $\varphi_{(P)}: T \rightarrow T_{(P)}$ such that the deformations $\mathcal{U}_{(P)} \rightarrow T$ and $W_{(P)} \times_{T_{(P)}} T$ are equivalent to each other in the sense of [4, Example (4.5)] by the versality there. In particular, $\left(\mathcal{U}_{(P)}, P^{\prime}\right)$ and $\left(W_{(P)} \times_{T_{(P)}} T, P\right)$ have a common étale neighborhood.

By the assumption (iii) and by Remark 4.3.2, there exist an algebraic $\Lambda$-scheme $S$ with a $\mathbb{k}$-rational point $b$ and a flat projective morphism $W \rightarrow S$ such that

- $W \rightarrow S$ is a deformation of $X$ with the reference point $b$,

- the completion of $\mathcal{O}_{S, b}$ is isomorphic to $\mathfrak{R}$, and

- $\mathfrak{X}$ is isomorphic over Spf $\mathfrak{R}$ to the formal completion of $W$ along the fiber over $b$.

Since $\operatorname{Spec} \mathcal{O}_{W, P}$ is a deformation of $\operatorname{Spec} \mathcal{O}_{X, P}$ over $S$ for any $P \in \operatorname{Sing} X$, after replacing $(S, b)$ with an étale neighborhood, we have a morphism $\phi_{(P)}: S \rightarrow T_{(P)}$ such that $(W, P)$ and $\left(W_{(P)} \times_{T_{(P)}} S, P\right)$ have a common étale neighborhood as in [4, Example (4.5)]. 
Let $\phi: S \rightarrow \prod_{P \in \operatorname{Sing} X} T_{(P)}$ be the morphism defined by $\left\{\phi_{(P)}\right\}$. Then, $\phi$ is smooth at $b$ by Theorem 4.6. Hence, we may assume that $\phi$ is smooth by replacing $S$ with an open neighborhood of $b$. Let $\varphi: T \rightarrow \prod_{P \in \operatorname{Sing} X} T_{(P)}$ be the morphism defined by $\left\{\varphi_{(P)}\right\}$. Since $\phi$ is smooth, after replacing $(T, o)$ with an étale neighborhood, we have a morphism $\psi: T \rightarrow S$ such that $\psi(o)=b$ and $\varphi=\phi \circ \psi$. Then, the base change $Z=W \times{ }_{S} T \rightarrow T$ satisfies the expected conditions.

Remark 4.7.1. The assumption (iii) above is satisfied if $\mathrm{H}^{2}\left(X, \mathcal{O}_{X}\right)=0$ (cf. [SGA1, Exp. III, Proposition 7.2]).

\section{Deformations of CERTAin ProjeCtive SuRfaCes With toric Singularities OF CLASS T}

We shall construct some algebraic deformations of a projective normal surface $X$ with toric singularities of class $\mathrm{T}$ under extra assumptions. We treat in Theorem 5.2 only deformations over $\mathbb{k}$, but in Theorem 5.4 , deformations over a complete discrete valuation ring with residue field $\mathbb{k}$. Here, we allow also rational double points on $X$ in Theorem 5.2 , As a corollary of Theorem 5.2, in Corollary 5.3, we shall give a correct proof of [40, Theorem 5.16] that any log del Pezzo surface of index two admits a $\mathbb{Q}$-Gorenstein smoothing to del Pezzo surfaces. Theorem 5.4 is applied to the study of the algebraic fundamental groups of smooth geometric fibers in Corollary 5.5 and Remark 5.5.1.

To begin with, we recall the following well-known result.

Lemma 5.1. Let $X$ be an affine algebraic $\mathbb{k}$-variety with a $\mathbb{k}$-rational point $P$ such that $X \backslash\{P\}$ is non-singular and $(X, P)$ is a local complete intersection singularity, i.e., the local ring $\mathcal{O}_{X, P}$ is a complete intersection. Then, there exist an affine open neighborhood $X^{\prime}$, an affine flat morphism $\mathcal{X} \rightarrow T$ over a non-singular curve $T$, and a $\mathbb{k}$-rational point $o \in T$ such that

(1) $\mathcal{X} \times_{T} O \simeq X^{\prime}$

(2) $\mathcal{X} \rightarrow T$ is smooth over $T \backslash\{o\}$.

In particular, the singularity $(X, P)$ admits a smoothing.

Proof. The local ring $\mathcal{O}_{X, P}$ is isomorphic to the localization of

$$
A=\mathbb{k}\left[\mathrm{x}_{1}, \ldots, \mathrm{x}_{n+l}\right] /\left(f_{1}, \ldots, f_{l}\right)
$$

at the origin $\left\{\mathrm{x}_{1}=\cdots=\mathrm{x}_{n+l}=0\right\}$ for a certain regular sequence $f_{1}, \ldots, f_{l}$, where $n=\operatorname{dim} X$. Hence, we may assume that $X=\operatorname{Spec} A$ and $P$ is the origin. For $1 \leq i \leq l$, let $F_{i}$ be the homogeneous polynomial in $\mathbb{k}\left[\mathrm{x}_{0}, \mathrm{x}_{1}, \ldots, \mathrm{x}_{n+l}\right]$ such that $F_{i}\left(1, \mathrm{x}_{1}, \ldots, \mathrm{x}_{n+l}\right)=f_{i}$ and $\mathrm{x}_{0} \nmid F_{i}$. Then, the complete intersection closed subscheme $\bar{X} \subset \mathbb{P}_{\mathbb{k}}^{n+l}$ defined by 
$\left\{F_{1}=\cdots=F_{l}=0\right\}$ is regarded as the closure of $X$, i.e., $\bar{X} \cap D_{+}\left(\mathrm{x}_{0}\right)=X$, where $D_{+}\left(\mathrm{x}_{0}\right)=\left\{\mathrm{x}_{0} \neq 0\right\}$. By Bertini's theorem, since $\mathbb{k}$ is algebraically closed, we can take homogeneous polynomials $G_{1}, \ldots, G_{l}$ with $\operatorname{deg} G_{i}=\operatorname{deg} F_{i}$ such that $\left\{G_{i}=0\right\}$ is nonsingular for all $1 \leq i \leq l$ and $\sum_{i=1}^{l}\left\{G_{i}=0\right\}$ is a simple normal crossing divisor. For $s$, $t \in \mathbb{k}$, let $H_{i}(s, t)$ be the divisor $\left\{s F_{i}+t G_{i}=0\right\}$. Then, there is an open neighborhood $T^{\prime} \subset \mathbb{P}^{1}$ of $(0: 1)$ such that, for any closed point $(s: t) \in T^{\prime}, H_{i}(s, t)$ is non-singular for all $1 \leq i \leq l$ and $\sum_{i=1}^{l} H_{i}(s, t)$ is a simple normal crossing divisor. Thus, for $T=$ $T^{\prime} \cup\{(1: 0)\}$,

$$
\mathcal{X}:=\left\{H_{1}(s, t)=\cdots=H_{l}(s, t)=0\right\} \cap D_{+}\left(\mathrm{x}_{0}\right) \rightarrow T
$$

is a desired morphism with $o=(1: 0) \in T$.

By [1, Corollary 6], we have:

Corollary 5.1.1. Any rational Gorenstein surface singularity (rational double point) admits a smoothing.

The following is our main technical tool for constructing desired surfaces of general type.

Theorem 5.2. Let $\mathbb{k}$ be an algebraically closed field. Let $X$ be a normal projective surface defined over $\mathbb{k}$ whose singularities are rational double points or toric singularities of class T. Assume that $X$ satisfies the following two conditions:

(i) $\mathrm{H}^{2}\left(X, \Theta_{X / \mathbb{k}}\right)=0$.

(ii) $\mathrm{H}^{2}\left(X, \mathcal{O}_{X}\right)=0$.

Then, there is a deformation $\mathcal{X} \rightarrow T$ of $X$ over a non-singular algebraic curve $T$ defined over $\mathbb{k}$ with a reference $\mathbb{k}$-rational point $o \in T$ such that:

(1) $\mathcal{X} \rightarrow T$ is a projective morphism and it is smooth over $T \backslash\{o\}$.

(2) $\mathcal{X}$ is normal, $r K_{\mathcal{X}}$ is Cartier, and $\left.\mathcal{O}_{\mathcal{X}}\left(r K_{\mathcal{X}}\right)\right|_{X} \simeq \mathcal{O}_{X}\left(r K_{X}\right)$ for the Gorenstein index $r$ of $X$.

In particular, after replacing $T$ with an open neighborhood of o, the following hold for any $\mathbb{k}$-rational point $t$ of $T \backslash\{o\}$ and the fiber $X_{t}:=\mathcal{X} \times_{T} t$ over $t$ :

(3) $X_{t}$ is a non-singular projective surface defined over $\mathbb{k}$.

(4) $\operatorname{dim} \mathrm{H}^{i}\left(X_{t}, \mathcal{O}_{X_{t}}\right)=\operatorname{dim} \mathrm{H}^{i}\left(X, \mathcal{O}_{X}\right)$ for all $i \geq 0$.

(5) $K_{X_{t}}^{2}=K_{X}^{2}$.

(6) $\mathrm{H}^{2}\left(X_{t}, \Theta_{X_{t} / \mathbb{k}}\right)=0$.

(7) If $K_{X}\left(\right.$ resp. $\left.-K_{X}\right)$ is ample, then so is $K_{X_{t}}$ (resp. $\left.-K_{X_{t}}\right)$.

(8) If $K_{X}\left(\right.$ resp. $\left.-K_{X}\right)$ is nef and big, then so is $K_{X_{t}}$ (resp. $\left.-K_{X_{t}}\right)$. 
Proof. In Theorem 5.2, it is enough to consider the deformation theory only in the case where $\Lambda=\mathbb{k}$. First of all, we shall prove (3)-(8) assuming (11) and (2). The assertions (3) and (4) follow from (11), the assumption (iii), and from the upper semi-continuity theorem for the flat morphism $\mathcal{X} \rightarrow T$. Since $(1 / 2) r^{2} K_{X}^{2}$ is the leading coefficient of the Hilbert polynomial $\chi\left(X, \mathcal{O}_{X}\left(m r K_{X}\right)\right)$ with respect to the variable $m$, (5) follows from (2) and the upper semi-continuity theorem for the flat morphism $\mathcal{X} \rightarrow T$. For (6) , let us consider the relative tangent sheaf $\Theta_{\mathcal{X} / T}:=\mathcal{H}_{o} m_{\mathcal{O}_{\mathcal{X}}}\left(\Omega_{\mathcal{X} / T}^{1}, \mathcal{O}_{\mathcal{X}}\right)$, where the canonical homomorphism $\Theta_{\mathcal{X} / T} \otimes_{\mathcal{O}_{\mathcal{X}}} \mathcal{O}_{X} \rightarrow \Theta_{X / \mathbb{k}}$ is an isomorphism outside $\operatorname{Sing} X$ but another canonical homomorphism $\Theta_{\mathcal{X} / T} \otimes_{\mathcal{O}_{\mathcal{X}}} \mathcal{O}_{X_{t}} \rightarrow \Theta_{X_{t} / \mathbb{k}}$ is an isomorphism. Then, we have $\mathrm{H}^{2}\left(X, \Theta_{\mathcal{X} / T} \otimes_{\mathcal{O}_{\mathcal{X}}} \mathcal{O}_{X}\right)=0$ by $\mathrm{H}^{2}\left(X, \Theta_{X / \mathbb{k}}\right)=0$. By the upper semi-continuity theorem applied to the sheaf $\Theta_{\mathcal{X} / T}$ flat over $T$ and by the base change isomorphism, we have the vanishing (6) .

The assertion (7) is derived from (2) and [EGA, III, Théoremè (4.7.1)]. The last assertion (8) is derived from also a general property. The detail is as follows (cf. [39, Chapter III, $\S 4 \mathrm{a}$, Problem]): Let $D$ be a Cartier divisor on $\mathcal{X}$ such that $D_{o}:=\left.D\right|_{X}$ is nef and big. It suffices to show that $D_{t}=\left.D\right|_{X_{t}}$ is also nef and big for any point $t$ in a neighborhood of $o$. Let $H$ be another Cartier divisor on $\mathcal{X}$ over $T$. Then, $D_{o} H_{o}=D_{t} H_{t}$, where $H_{t}:=\left.H\right|_{X_{t}}$ by considering the Hilbert polynomial $\chi\left(X_{t},\left.\mathcal{O}_{\mathcal{X}}\left(m_{1} H+m_{2} D\right)\right|_{X_{t}}\right)$ of two variables $m_{1}, m_{2}$. In particular, $D_{t}^{2}=D_{o}^{2}>0$ and $D_{t} H_{t}>0$ for an ample divisor $H_{t}$. Hence, $D_{t}$ is big. Thus, by replacing $T$ with an affine open neighborhood of $o$, we have an effective divisor $G$ on $\mathcal{X}$ such that $m D \sim G$ for some $m>0$. Here, $D_{t}$ is nef if and only if $D_{t} C \geq 0$ for any irreducible component $C$ of $G_{t}=\left.G\right|_{X_{t}}$. By eliminating the vertical components of $G$, we may assume that any irreducible component $G_{\lambda}$ of $G$ dominates $T$. Then, $0 \leq D_{o} G_{\lambda, o}=D_{t} G_{\lambda, t}$, where $G_{\lambda, t}=\left.G_{\lambda}\right|_{X_{t}}$. Replacing $T$ by the Stein factorization of $G_{\lambda} \rightarrow T$ if necessary, we see that $D_{t}$ is nef for a general point of $T$.

We shall construct a deformation $\mathcal{X} \rightarrow T$ satisfying (11) and (2) by applying Theorem 4.7. Note that the assumption (ii) of Theorem 4.7 is identical to (ii) above and the other assumption (ii) of Theorem 4.7 is satisfied by (iii) above (cf. Remark 4.7.1). For a singular point $P$, we shall construct a deformation $\mathcal{U}_{(P)} \rightarrow T$ of an affine étale neighborhood of $(X, P)$ over a non-singular curve $T$ as follows. If $P$ is a rational double point, then we set $\mathcal{U}_{(P)} \rightarrow T$ to be a deformation obtained in Lemma 5.1 (cf. Corollary 5.1.1), which is a smoothing of $(X, P)$. Note that $\mathcal{U}_{(P)}$ is normal and Gorenstein. If $(X, P)$ is a toric singularity of class $\mathrm{T}$, then, by Theorem 2.6, there is an affine étale neighborhood $\left(U_{(P)}, P^{\prime}\right)$ of $(X, P)$ which is also an étale neighborhood of $\left(V_{(P)}, \mathbf{0}\right)$ for an affine toric surface $V_{(P)}$ with the closed orbit $\mathbf{0}$. In this case, by applying Theorem 3.8 to $V_{(P)}$ and Lemma 4.4 .2 to $\left(U_{(P)}, P^{\prime}\right) \rightarrow\left(V_{(P)}, \mathbf{0}\right)$, we have a deformation $\mathcal{U}_{(P)} \rightarrow T$ of $U_{(P)}$ with a $\mathbb{k}$-rational reference point $o$ such that 
- $\mathcal{U}_{(P)} \rightarrow T$ is smooth over $T \backslash\{o\}$,

- $\mathcal{U}_{(P)}$ is normal, $r K_{\mathcal{U}_{(P)}}$ is Cartier, and

$$
\left.\mathcal{O}_{\mathcal{U}_{(P)}}\left(r K_{\mathcal{U}_{(P)}}\right)\right|_{U_{(P)}} \simeq \mathcal{O}_{U_{(P)}}\left(r K_{U_{(P)}}\right)
$$

for the Gorenstein index $r$ of $(X, P)$.

Note that in the construction above, we can take a common non-singular curve $T$ and a reference point $o$.

Applying Theorem 4.7 to $\mathcal{U}_{(P)} \rightarrow T$ for any $P \in \operatorname{Sing} X$, after replacing $(T, o)$ with an étale neighborhood, we have a deformation $\mathcal{X} \rightarrow T$ of $X$ satisfying the required conditions (11) and (2). Thus, we are done.

We shall correct the proof of [40, Theorem 5.16] concerning $\mathbb{Q}$-Gorenstein smoothings of log del Pezzo surfaces of index two by proving the following stronger assertion as an application of Theorem 5.2 .

Corollary 5.3. Let $X$ be a log del Pezzo surface of index two over an algebraically closed field $\mathbb{k}$, i.e., $X$ is a normal projective surface, $X$ has only log-terminal singularities, $-K_{X}$ is an ample non-Cartier divisor, and $2 K_{X}$ is Cartier (cf. [40, Definition 3.2]). Then, there is a projective deformation $\mathcal{X} \rightarrow T$ of $X$ over a non-singular curve $T$ over $\mathbb{k}$ such that

- $\mathcal{X} \rightarrow T$ is smooth outside $X$,

- $2 K_{\mathcal{X}}$ is Cartier, and

- any closed fiber of $\mathcal{X} \rightarrow T$ other than $X$ is a del Pezzo surface with $K^{2}=K_{X}^{2}$.

Proof. A singular point of $X$ is either a rational double point or a singular point of type $\mathrm{K}_{n}$ (cf. [40, Lemma 4.15]), where $\mathrm{K}_{n}$ is just the toric singularity of type $\frac{1}{4 n}(1,2 n-1)$ : this is of type $T(n, 2,1)$ (cf. Definition 3.2). We have $\mathrm{H}^{2}\left(X, \mathcal{O}_{X}\right)=\mathrm{H}^{0}\left(X, \mathcal{O}_{X}\left(K_{X}\right)\right)^{\vee}=0$ by the Serre duality theorem, since $-K_{X}$ is ample. Hence, by Theorem 5.2, it suffices to show $\mathrm{H}^{2}\left(X, \Theta_{X / \mathbb{k}}\right)=0$, or equivalently, $\mathrm{H}^{0}\left(X, \mathcal{H}_{o m_{\mathcal{O}_{X}}}\left(\Theta_{X / \mathbb{k}}, \mathcal{O}_{X}\left(K_{X}\right)\right)\right)=0$ by Serre duality. We know that $\mathrm{H}^{0}\left(X, \mathcal{O}_{X}\left(-K_{X}\right)\right) \neq 0$. In fact, for the minimal resolution $\mu: M \rightarrow X$ of singularities, we have

$$
\begin{aligned}
& \mathrm{H}^{0}\left(X, \mathcal{O}_{X}\left(-K_{X}\right)\right) \simeq \mathrm{H}^{0}\left(M, \mathcal{O}_{M}\left(K_{M}+\mu^{*}\left(-2 K_{X}\right)\right)\right), \\
& \mathrm{H}^{2}\left(M, \mathcal{O}_{M}\left(K_{M}+\mu^{*}\left(-2 K_{X}\right)\right)\right) \simeq \mathrm{H}^{0}\left(M, \mu^{*} \mathcal{O}_{X}\left(2 K_{X}\right)\right)^{\vee}=0, \quad \text { and } \\
& \operatorname{dim} \mathrm{H}^{0}\left(M, \mathcal{O}_{M}\left(K_{M}+\mu^{*}\left(-2 K_{X}\right)\right)\right) \geq \chi\left(M, \mathcal{O}_{M}\left(K_{M}+\mu^{*}\left(-2 K_{X}\right)\right)\right) \\
& \quad=\frac{1}{2}\left(K_{M}+\mu^{*}\left(-2 K_{X}\right)\right) \mu^{*}\left(-2 K_{X}\right)+1=K_{X}^{2}+1>0 .
\end{aligned}
$$

Taking a non-zero section of $\mathcal{O}_{X}\left(-K_{X}\right)$, we obtain an injection $\mathcal{O}_{X}\left(K_{X}\right) \hookrightarrow \mathcal{O}_{X}$, and hence an injection

$$
\mathcal{H} \operatorname{Hom}_{\mathcal{O}_{X}}\left(\Theta_{X / \mathbb{k}}, \mathcal{O}_{X}\left(K_{X}\right)\right) \hookrightarrow \mathcal{H} \operatorname{om}_{\mathcal{O}_{X}}\left(\Theta_{X / \mathbb{k}}, \mathcal{O}_{X}\right) \simeq\left(\Omega_{X / \mathbb{k}}^{1}\right)^{\vee \vee}
$$


where the right sheaf is isomorphic to $\mu_{*} \Omega_{M / \mathbb{k}}^{1}$ by Proposition 2.11,(3). Since $M$ is rational,

$$
\mathrm{H}^{0}\left(X, \mathcal{H} \operatorname{Hom}_{\mathcal{O}_{X}}\left(\Theta_{X / \mathbb{k}}, \mathcal{O}_{X}\left(K_{X}\right)\right)\right) \subset \mathrm{H}^{0}\left(M, \Omega_{M / \mathbb{k}}^{1}\right)=0 .
$$

Thus, we are done.

Theorem 5.4. Let $\Lambda$ be a complete discrete valuation ring with an algebraically closed residue field $\mathbb{k}$. Let $X_{\Lambda} \rightarrow \operatorname{Spec} \Lambda$ be a flat projective morphism satisfying the following two conditions:

(i) The closed fiber $X=X_{\mathbb{k}}$ is a normal projective surface with only toric singularities of class $T$ satisfying $\mathrm{H}^{2}\left(X, \Theta_{X / \mathbb{k}}\right)=\mathrm{H}^{2}\left(X, \mathcal{O}_{X}\right)=0$.

(ii) For any singular point $P$ of $X$, let $\left(n_{(P)}, q_{(P)}\right)$ be the type of the toric singularity. Then, there exist an affine neighborhood $Y_{(P)}$ of $P$ in $X_{\Lambda}$ and two prime divisors $B_{1(P)}, B_{2(P)}$ on $Y_{(P)}$ containing $P$ such that $\left(Y_{(P)}, B_{1(P)}, B_{2(P)}\right)$ satisfies the condition $C\left(n_{(P)}, q_{(P)}\right)^{\prime}$ over $\operatorname{Spec} \Lambda$ (cf. Definition 2.1).

Then, there exist an algebraic deformation $Z \rightarrow T$ of $X$ over an algebraic smooth $\Lambda$ scheme $T$ of relative dimension one, a $\mathbb{k}$-rational point $o \in T$, and a section $\sigma: \operatorname{Spec} \Lambda \rightarrow$ T such that:

(1) $\sigma\left(\mathfrak{m}_{\Lambda}\right)=o$ and $Z \times_{T, \sigma} \operatorname{Spec} \Lambda \simeq X_{\Lambda}$.

(2) $Z$ is smooth over $T \backslash \sigma(\operatorname{Spec} \Lambda)$.

(3) $Z$ is normal, $r K_{Z}$ is Cartier, and $\left.\mathcal{O}_{Z}\left(r K_{Z}\right)\right|_{X_{\Lambda}} \simeq \mathcal{O}_{X_{\Lambda}}\left(r K_{X_{\Lambda}}\right)$ for the Gorenstein index $r$ of $X$.

Proof. By Theorems 2.7, 3.8 and by Lemma 4.4.2, we have an algebraic smooth $\Lambda$-scheme $T$ of relative dimension one, a $\mathbb{k}$-rational reference point $o \in T$, a section $\sigma: \operatorname{Spec} \Lambda \rightarrow T$ with $\sigma\left(\mathfrak{m}_{\Lambda}\right)=o$, and a flat family $V_{(P)} \rightarrow T$ of normal affine surfaces for any singular point $P$ of $X$ such that

(V1) $V_{(P)} \times_{T, \sigma} \operatorname{Spec} \Lambda \simeq Y_{(P)}$,

$(V 2) V_{(P)} \rightarrow T$ is smooth over $T \backslash \sigma(\operatorname{Spec} \Lambda)$,

$(V 3) V_{(P)}$ is normal, $r_{P} K_{V_{(P)}}$ is Cartier with $\left.\mathcal{O}_{V_{(P)}}\left(r_{P} K_{V_{(P)}}\right)\right|_{Y_{(P)}} \simeq \mathcal{O}_{Y_{(P)}}\left(r_{P} K_{Y_{(P)}}\right)$ for the Gorenstein index $r_{P}$ of the toric singularity $P \in X$.

In fact, $\left(Y_{(P)}, P\right)$ is an étale neighborhood of an affine toric surface at the closed orbit by Theorem 2.7, and the toric surface admits a deformation satisfying conditions (1)(3) of Theorem 3.8 since it is of class $\mathrm{T}$, and finally by Lemma 4.4 .2 , we can lift the deformation to that of $Y_{(P)}$. Here, the section in Theorem 3.8 induces the section $\sigma$, since $\Lambda$ is a complete discrete valuation ring.

Let $\mathfrak{X} \rightarrow$ Spf $\mathfrak{R}$ be the formal deformation associated with a hull $(\mathfrak{R}, \xi)$ of $\operatorname{Def}_{X}$. By the assumption (ii), we can find an algebraization $W \rightarrow S$ of $\mathfrak{X} \rightarrow$ Spf $\mathfrak{R}$ as in the proof 
of Theorem 4.7 by Remark 4.7.1. Here, $W \rightarrow S$ is a projective flat morphism over an algebraic $\Lambda$-scheme $S$, the fiber over a $\mathbb{k}$-rational point $b \in S$ is isomorphic to $X$, the completion of $\mathcal{O}_{S, b}$ is isomorphic to $\mathfrak{R}$, and the formal completion of $W$ along $X$ is isomorphic to $\mathfrak{X}$. Since $X_{\Lambda} \rightarrow \operatorname{Spec} \Lambda$ is a deformation of $X$ with reference point $\mathfrak{m}_{\Lambda}$, we have a surjection $\mathfrak{R} \rightarrow \Lambda$ such that $\mathfrak{X} \times_{\operatorname{Spf} \Re} \operatorname{Spf} \Lambda$ is isomorphic to the formal completion of $X_{\Lambda}$ along $X$. Hence, for the induced section $\sigma_{1}: \operatorname{Spec} \Lambda \rightarrow \operatorname{Spec} \mathfrak{R} \rightarrow \operatorname{Spec} \mathcal{O}_{S, b} \rightarrow S$, we have an isomorphism $W \times_{S, \sigma_{1}} \operatorname{Spec} \Lambda \simeq X_{\Lambda}$ by [EGA, III, Théorème (5.4.1)].

Let $W_{(P)} \rightarrow T_{(P)}$ be an algebraization of the formal deformation associated with a hull of $\operatorname{Def}_{(X, P)}$ as in the proof of Theorem 4.7. After replacing $(S, b)$ with an étale neighborhood and replacing $(T, o)$ with an étale neighborhood, we have morphisms $\phi: S \rightarrow$ $\prod_{P \in \operatorname{Sing} X} T_{(P)}$ and $\varphi: T \rightarrow \prod_{P \in \operatorname{Sing} X} T_{(P)}$ such that $\varphi \circ \sigma=\phi \circ \sigma_{1}(\mathrm{cf}$. (V1) ) and that, for any $P \in \operatorname{Sing} X$,

- $(W, P)$ and $\left(W_{(P)} \times_{T_{(P)}} S, P\right)$ have a common étale neighborhood,

- $\left(V_{(P)}, P\right)$ and $\left(W_{(P)} \times_{T_{(P)}} T, P\right)$ have a common étale neighborhood.

Let $S_{T} \rightarrow T$ be the base change of $\phi$ by $\varphi$. By replacing $T$ with an open neighborhood of $o$, we may assume that $S_{T} \rightarrow T$ is smooth, since $\phi$ is smooth at $b$ by Theorem 4.6. Now, we have a section $\sigma^{\prime}=\left(\sigma, \sigma_{1}\right): \operatorname{Spec} \Lambda \rightarrow S_{T}$. Then, after replacing $(T, o)$ with an étale neighborhood, we have a section $\psi: T \rightarrow S_{T}$ such that $\psi \circ \sigma=\sigma^{\prime}$. In fact, $S_{T}$ is étale over $\mathbb{A}_{T}^{k}:=\mathbb{A}_{\Lambda}^{k} \times_{\operatorname{Spec} \Lambda} T$ for some $k$, and $\sigma^{\prime}$ induces a section of $\mathbb{A}_{\Lambda}^{k}$ over Spec $\Lambda$. Here, we may assume that the section is defined by $t_{1}=\cdots=t_{k}=0$ for $\mathbb{A}_{\Lambda}^{k}=\operatorname{Spec} \Lambda\left[t_{1}, \ldots, t_{k}\right]$. The closed subscheme of $\mathbb{A}_{T}^{k}$ defined by $\mathrm{t}_{1}=\cdots=\mathrm{t}_{k}=0$ is isomorphic to $T$. Hence a connected component of the pullback of the closed subscheme by $S_{T} \rightarrow \mathbb{A}_{T}^{k}$ gives a desired étale neighborhood. Let $Z \rightarrow T$ be the base change of $W \rightarrow S$ by $T \rightarrow S_{T} \rightarrow S$. Then, $Z \rightarrow T$ is a deformation of $X$ with reference point $o$ satisfying the condition (1), and $(Z, P)$ and $\left(V_{(P)}, P\right)$ have a common étale neighborhood for any $P \in \operatorname{Sing} X$. Hence, the other conditions (2) and (3) are derived from ( $(\underline{V 2})$ and $(\underline{V 3})$, and we have finished the proof.

Corollary 5.5 (Fundamental group). Let $X_{\Lambda} \rightarrow \operatorname{Spec} \Lambda$ be the flat projective morphism in Theorem 5.4 satisfying the two conditions (ii) and (iii). Assume that the field of fractions of $\Lambda$ is of characteristic zero. Let $X$ be the closed fiber of $X_{\Lambda} \rightarrow \operatorname{Spec} \Lambda$. Let $\mathbb{K}$ be an algebraically closed field containing $\Lambda$ and let $X_{\mathbb{K}}$ be the fiber product $X_{\Lambda} \times_{\operatorname{Spec} \Lambda} \operatorname{Spec} \mathbb{K}$. Then, $\mathrm{H}^{2}\left(X_{\mathbb{K}}, \mathcal{O}_{X_{\mathbb{K}}}\right)=\mathrm{H}^{2}\left(X_{\mathbb{K}}, \Theta_{X_{\mathbb{K}} / \mathbb{K}}\right)=0$. Moreover, there exist a deformation $\mathcal{X} \rightarrow C$ of $X$ over a non-singular algebraic curve $C$ defined over $\mathbb{k}$, and a deformation $\mathcal{Y} \rightarrow D$ of $X_{\mathbb{K}}$ over a non-singular algebraic curve $D$ defined over $\mathbb{K}$ such that $\mathcal{X} \rightarrow C$ and $\mathcal{Y} \rightarrow D$ satisfy the conditions corresponding to (11) and (2) of Theorem 5.2 and that there is a 
surjection

$$
\pi_{1}^{\mathrm{alg}}\left(Y_{d}\right) \rightarrow \pi_{1}^{\mathrm{alg}}\left(X_{c}\right)
$$

of algebraic fundamental groups for any smooth fibers $X_{c}$ and $Y_{d}$ of $\mathcal{X} \rightarrow C$ and $\mathcal{Y} \rightarrow D$ over closed points $c \in C$ and $d \in D$, respectively.

Proof. First, we shall show: $\mathrm{H}^{2}\left(X_{\mathbb{K}}, \mathcal{O}_{X_{\mathbb{K}}}\right)=\mathrm{H}^{2}\left(X_{\mathbb{K}}, \Theta_{X_{\mathbb{K}} / \mathbb{K}}\right)=0$. Since $\mathrm{H}^{2}\left(X, \mathcal{O}_{X}\right)=0$, we have $\mathrm{H}^{2}\left(X_{\Lambda}, \mathcal{O}_{X_{\Lambda}}\right)=0$ by the upper semi-continuity theorem for the flat morphism $X_{\Lambda} \rightarrow \operatorname{Spec} \Lambda$, and $\mathrm{H}^{2}\left(X_{\mathbb{K}}, \mathcal{O}_{X_{\mathbb{K}}}\right)=0$ by the flat base change isomorphism $\mathrm{H}^{2}\left(X_{\Lambda}, \mathcal{O}_{X_{\Lambda}}\right) \otimes_{\Lambda}$ $\mathbb{K} \simeq \mathrm{H}^{2}\left(X_{\mathbb{K}}, \mathcal{O}_{X_{\mathbb{K}}}\right)$. The vanishing of $\mathrm{H}^{2}\left(X_{\mathbb{K}}, \Theta_{X_{\mathbb{K}} / \mathbb{K}}\right)$ is shown as in the proof of Theorem 5.2,(6) : For the relative tangent sheaf $\Theta_{X_{\Lambda} / \Lambda}:=\mathcal{H}_{o} m_{\mathcal{O}_{X_{\Lambda}}}\left(\Omega_{X_{\Lambda} / \Lambda}^{1}, \mathcal{O}_{X_{\Lambda}}\right)$, the canonical homomorphism $\Theta_{X_{\Lambda} / \Lambda} \otimes_{\mathcal{O}_{X_{\Lambda}}} \mathcal{O}_{X} \rightarrow \Theta_{X / \mathbb{k}}$ is an isomorphism outside Sing $X$ but another canonical homomorphism $\Theta_{X_{\Lambda} / \Lambda} \otimes_{\Lambda} \mathbb{K} \rightarrow \Theta_{X_{\mathbb{K}} / \mathbb{K}}$ is an isomorphism. Thus, we have $\mathrm{H}^{2}\left(X, \Theta_{X_{\Lambda} / \Lambda} \otimes_{\mathcal{O}_{X_{\Lambda}}} \mathcal{O}_{X}\right)=0$ by $\mathrm{H}^{2}\left(X, \Theta_{X / \mathbb{k}}\right)=0$. By the upper semi-continuity theorem applied to the sheaf $\Theta_{X_{\Lambda} / \Lambda}$ flat over $\Lambda$ and by the base change isomorphism, we have the vanishing $\mathrm{H}^{2}\left(X_{\mathbb{K}}, \Theta_{X_{\mathbb{K}} / \mathbb{K}}\right)=0$.

Second, we shall define $\mathcal{X} \rightarrow C$ and $\mathcal{Y} \rightarrow D$. Let $Z \rightarrow T$ be the deformation of $X$ obtained in Theorem 5.4. By the surjection $\Lambda \rightarrow \mathbb{k}$ and the injection $\Lambda \hookrightarrow \mathbb{K}$, we define

$$
\begin{aligned}
& \mathcal{X}:=Z \times_{\operatorname{Spec} \Lambda} \operatorname{Spec} \mathbb{k} \rightarrow C:=T \times_{\operatorname{Spec} \Lambda} \operatorname{Spec} \mathbb{k}, \quad \text { and } \\
& \mathcal{Y}:=Z \times_{\operatorname{Spec} \Lambda} \operatorname{Spec} \mathbb{K} \rightarrow D:=C \times_{\operatorname{Spec} \Lambda} \operatorname{Spec} \mathbb{K} .
\end{aligned}
$$

Then, $\mathcal{X} \rightarrow C$ is a deformation of $X$ with reference point $o=C \cap \sigma(\operatorname{Spec} \Lambda)$, and $\mathcal{Y} \rightarrow D$ is a deformation of $X_{\mathbb{K}}$ with the reference point $o_{\mathbb{K}}:=D \times_{Z} \sigma(\operatorname{Spec} \Lambda)$. Moreover, these deformations satisfy the conditions corresponding to (11) and (2) of Theorem 5.2 .

Finally, we shall compare several algebraic fundamental groups using results in [SGA1, Exp. X] concerning with Grothendieck's specialization theorem [SGA1, Exp. X, Corollaire 2.4, Théorème 3.8]. Let $\mathbb{k}_{1}$ be the algebraic closure of the function field $\mathbb{k}(C)$ of $C$ and set $\mathcal{X}_{\mathrm{k}_{1}}$ to be the fiber product $\mathcal{X} \times_{C} \operatorname{Spec} \mathbb{k}_{1}$. Then, for any smooth closed fiber $X_{c}$ of $\mathcal{X} \rightarrow C$, we have a surjection

$$
\pi_{1}^{\text {alg }}\left(\mathcal{X}_{\mathbb{k}_{1}}\right) \rightarrow \pi_{1}^{\text {alg }}\left(X_{c}\right)
$$

by [SGA1, Exp. X, Corollaire 2.4, Théorème 3.8].

Let $\mathbb{K}_{1}$ be an algebraically closed field containing the function field $\mathbb{K}(D)$ of $D$, and let $\mathcal{Y}_{\mathbb{K}_{1}}$ be the fiber product $\mathcal{Y} \times_{D}$ Spec $\mathbb{K}_{1}$. The geometric generic points Spec $\mathbb{K}_{1} \rightarrow$ $D \rightarrow T$ and Spec $\mathbb{k}_{1} \rightarrow C \rightarrow T$ are lying on the open subset $T \backslash \sigma(\operatorname{Spec} \Lambda)$, and the corresponding geometric fibers of $Z \rightarrow T$ are $\mathcal{Y}_{\mathbb{K}_{1}}$ and $\mathcal{X}_{\mathbb{k}_{1}}$, respectively. By [SGA1, Exp. X, Corollaire 2.4, Théorème 3.8], we have a surjection

$$
\pi_{1}^{\mathrm{alg}}\left(\mathcal{Y}_{\mathbb{K}_{1}}\right) \rightarrow \pi_{1}^{\mathrm{alg}}\left(\mathcal{X}_{\mathbb{k}_{1}}\right)
$$


Since $\operatorname{char}(\mathbb{K})=0$, by [SGA1, Exp. X, Théoremè 3.8, Corollaire 3.9], we have an isomorphism

$$
\pi_{1}^{\mathrm{alg}}\left(\mathcal{Y}_{\mathbb{K}_{1}}\right) \simeq \pi_{1}^{\mathrm{alg}}\left(Y_{d}\right)
$$

for any smooth closed fiber $Y_{d}$ of $\mathcal{Y} \rightarrow D$. Thus, we have a desired surjection $\pi_{1}^{\text {alg }}\left(Y_{d}\right) \rightarrow$ $\pi_{1}^{\text {alg }}\left(X_{c}\right)$ from (V-1)-(

Remark 5.5.1. The calculation of $\pi_{1}^{\text {alg }}\left(Y_{d}\right)$ in Corollary 5.5 is reduced to the case over the complex number field $\mathbb{C}$, as follows. Let $\mathbb{K}, X_{\mathbb{K}}$, and $\mathcal{Y} \rightarrow D$ be as in Corollary 5.5. Here, $\mathcal{Y} \rightarrow D$ is a deformation of $X_{\mathbb{K}}$ with a reference $\mathbb{K}$-rational point $b:=o_{\mathbb{K}} \in D$ as in the proof of Corollary 5.5. Then, there is a finitely generated field $\mathbb{K}_{0}$ over the field $\mathbb{Q}$ of rational numbers such that $X_{\mathbb{K}}, \mathcal{Y} \rightarrow D, o_{\mathbb{K}} \in D, d \in D \backslash\{b\}$, and every point of Sing $X_{\mathbb{K}}=\left\{P_{1}, \ldots, P_{k}\right\}$ descend to over $\mathbb{K}_{0}$. Namely, there exist algebraic schemes $X_{0}$ and $D_{0}$ over Spec $\mathbb{K}_{0}, \mathbb{K}_{0}$-rational points $b_{0}$ and $d_{0}$ of $D_{0}, \mathbb{K}$-rational points $P_{1,0}, \ldots, P_{k, 0}$ of $X_{0}$, and a morphism $\mathcal{Y}_{0} \rightarrow D_{0}$ such that

$$
\begin{gathered}
X_{\mathbb{K}} \simeq X_{0} \times_{\text {Spec } \mathbb{K}_{0}} \operatorname{Spec} \mathbb{K}, \quad D \simeq D_{0} \times_{\text {Spec } \mathbb{K}_{0}} \operatorname{Spec} \mathbb{K}, \\
(b \in D) \simeq\left(b_{0} \in D_{0}\right) \times_{\text {Spec } \mathbb{K}_{0}} \operatorname{Spec} \mathbb{K}, \quad(d \in D) \simeq\left(d_{0} \in D_{0}\right) \times_{\text {Spec } \mathbb{K}_{0}} \operatorname{Spec} \mathbb{K}, \\
\left(P_{i} \in X\right) \simeq\left(P_{i, 0} \in X_{0}\right) \times_{\text {Spec } \mathbb{K}_{0}} \operatorname{Spec} \mathbb{K} \quad(1 \leq i \leq k), \\
(\mathcal{Y} \rightarrow D) \simeq\left(\mathcal{Y}_{0} \rightarrow D_{0}\right) \times_{\text {Spec } \mathbb{K}_{0}} \operatorname{Spec} \mathbb{K},
\end{gathered}
$$

with the following properties (cf. [EGA, IV, Propositions (2.5.1), (2.7.1), (6.7.4), Corollaire (2.7.2)]):

- $X_{0}$ is a normal projective integral $\mathbb{K}_{0}$-scheme, and $X_{0} \backslash\left\{P_{i, 0}\right\}_{1 \leq i \leq k}$ is smooth over $\operatorname{Spec} \mathbb{K}_{0}$.

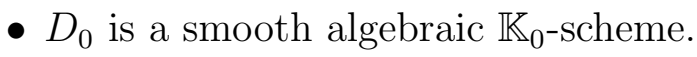

- $\mathcal{Y}_{0}$ is normal and integral.

- $\mathcal{Y}_{0} \rightarrow D_{0}$ is a projective flat morphism whose fiber over $b_{0}$ is identified with $X_{0}$.

- $\mathcal{Y}_{0} \rightarrow D_{0}$ is smooth on $\mathcal{Y}_{0}^{\circ}:=\mathcal{Y}_{0} \backslash\left\{P_{i, 0}\right\}_{1 \leq i \leq k}$.

Moreover, $r K_{\mathcal{Y}_{0} / D_{0}}$ is Cartier for the relative canonical divisor $K_{\mathcal{Y}_{0} / D_{0}}$ and for the index $r$ of $X$, and there is an isomorphism

$$
\mathcal{O}_{\mathcal{Y}_{0}}\left(r K_{\mathcal{Y}_{0} / D_{0}}\right) \simeq j_{*}\left(\omega_{\mathcal{Y}_{0}^{\circ} / D_{0}}^{\otimes r}\right)
$$

for the relative dualizing sheaf $\omega_{\mathcal{Y}_{0}^{\circ} / D_{0}}$ where $j: \mathcal{Y}_{0}^{\circ} \hookrightarrow \mathcal{Y}_{0}$ denotes the open immersion. These properties are also derived from the corresponding properties on $\mathcal{Y} \rightarrow D$. Note that the fiber $Y_{d}$ of $\mathcal{Y} \rightarrow D$ over $d$ is the base change of the fiber $Y_{d_{0}}$ of $\mathcal{Y}_{0} \rightarrow D_{0}$ over $d_{0}$ 
by Spec $\mathbb{K} \rightarrow \operatorname{Spec} \mathbb{K}_{0}$. Taking a field extension $\mathbb{K}_{0} \subset \mathbb{C}$, we set

$$
\begin{gathered}
X_{\mathbb{C}}:=X_{0} \times_{\operatorname{Spec} \mathbb{K}_{0}} \operatorname{Spec} \mathbb{C}, \quad D_{\mathbb{C}}:=D_{0} \times_{\operatorname{Spec} \mathbb{K}_{0}} \operatorname{Spec} \mathbb{C}, \\
\left(b_{\mathbb{C}} \in D_{\mathbb{C}}\right):=\left(b_{0} \in D_{0}\right) \times_{\operatorname{Spec} \mathbb{K}_{0}} \operatorname{Spec} \mathbb{C}, \quad\left(d_{\mathbb{C}} \in D_{\mathbb{C}}\right):=\left(d_{0} \in D_{0}\right) \times_{\operatorname{Spec} \mathbb{K}_{0}} \operatorname{Spec} \mathbb{C}, \\
\left(P_{i, \mathbb{C}} \in D_{\mathbb{C}}\right):=\left(P_{i, 0} \in D_{0}\right) \times_{\operatorname{Spec} \mathbb{K}_{0}} \operatorname{Spec} \mathbb{C}, \quad(1 \leq i \leq k), \\
\left(\mathcal{Y}_{\mathbb{C}} \rightarrow D_{\mathbb{C}}\right):=\left(\mathcal{Y}_{0} \rightarrow D_{0}\right) \times_{\text {Spec } \mathbb{K}_{0}} \operatorname{Spec} \mathbb{C} .
\end{gathered}
$$

By considering also the descent of the minimal resolution of singularities of $X$, we may assume that $X_{\mathbb{C}}$ has only toric singularities of class $\mathrm{T}$. In fact, the exceptional locus over $P_{i, \mathbb{C}}$ of the minimal resolution of $X_{\mathbb{C}}$ is the linear chain of rational curves with the same self-intersection numbers as that for $P_{i}$; thus $\left(X_{\mathbb{C}}, P_{i, \mathbb{C}}\right)$ is a toric singularity by Theorem 2.6. Then, $\mathcal{Y}_{\mathbb{C}} \rightarrow D_{\mathbb{C}}$ is a $\mathbb{Q}$-Gorenstein smoothing of $X_{\mathbb{C}}$ in the sense of [26, Section 3] (cf. [26, Corollary 3.6]), since $\mathcal{Y}_{\mathbb{C}}$ is $\mathbb{Q}$-Gorenstein. For the smooth fiber $Y_{\mathbb{C}, d_{\mathbb{C}}}$ of $\mathcal{Y}_{\mathbb{C}} \rightarrow D_{\mathbb{C}}$ over $d_{\mathbb{C}}$, we have an isomorphism

$$
\pi_{1}^{\mathrm{alg}}\left(Y_{d}\right) \simeq \pi_{1}^{\mathrm{alg}}\left(Y_{\mathbb{C}, d_{\mathbb{C}}}\right)
$$

by [SGA1, Exp. X, Corollaire 1.8].

\section{Simply CONneCted Surfaces of General Type With $p_{g}=q=0$}

We apply the results in Sections 25 to construct algebraically simply connected surfaces $\mathbb{S}$ of general type with $p_{g}=q=0$ and $1 \leq K^{2} \leq 4$ which is defined over the given algebraically closed field $\mathbb{k}$, where

$$
p_{g}=p_{g}(\mathbb{S})=\operatorname{dim} \mathrm{H}^{0}\left(\mathbb{S}, \mathcal{O}_{\mathbb{S}}\left(K_{\mathbb{S}}\right)\right)=\operatorname{dim} \mathrm{H}^{2}\left(\mathbb{S}, \mathcal{O}_{\mathbb{S}}\right), \quad q=q(\mathbb{S})=\operatorname{dim} \mathrm{H}^{1}\left(\mathbb{S}, \mathcal{O}_{\mathbb{S}}\right)
$$

and $K=K_{\mathbb{S}}$ denotes the canonical divisor. An outline of our method is as follows. We first construct a normal projective rational surface $X$ with only toric singularities of class $\mathrm{T}$ satisfying the following conditions:

- $K_{X}$ is nef and big (or ample),

- $K_{X}^{2}$ equals the given number $K^{2}>0$,

- $\mathrm{H}^{2}\left(X, \Theta_{X}\right)=0$.

Note that $\mathrm{H}^{i}\left(X, \mathcal{O}_{X}\right)=0$ for any $i>0$ since $X$ is rational and has only rational singularities. For the construction of $X$, we follow the method used in [33], [42], and [43], which is however considered over the field $\mathbb{C}$ of complex numbers. By Theorem 5.2, we have a projective deformation $\mathcal{X} \rightarrow T$ of $X$ over a non-singular curve $T$ defined over $\mathbb{k}$ satisfying the conditions (11) and (2) of Theorem 5.2. Here, a general closed smooth fiber $X_{t}$ of $\mathcal{X} \rightarrow T$ is a non-singular projective surface $\mathbb{S}$ having the following properties:

- $q(\mathbb{S})=p_{g}(\mathbb{S})=0$ and $\chi\left(\mathbb{S}, \mathcal{O}_{\mathbb{S}}\right)=1$. 
- $\mathbb{S}$ is a minimal surface of general type with $K_{\mathbb{S}}^{2}=K^{2}$.

- $\mathrm{H}^{2}\left(\mathbb{S}, \Theta_{\mathbb{S} / \mathbb{k}}\right)=0$. In particular, $\mathbb{S}$ is liftable to characteristic zero.

For the second condition above, note that $K_{X_{t}}$ is ample if $K_{X}$ is. If $X_{t}$ is algebraically simply connected, then this is one of the surfaces what we want to get. In order to construct a simply connected one, we select the deformation $\mathcal{X} \rightarrow T$ by considering a lifting problem to characteristic zero. Namely, we construct $X$ as the closed fiber of a flat family $X_{\Lambda} \rightarrow$ Spec $\Lambda$ for a complete discrete valuation ring $\Lambda$ of mixed characteristic with the residue field $\mathbb{k}$ satisfying the assumptions (11) and (2) of Theorem 5.4. Then, by Corollary [5.5, we have a deformation $\mathcal{X} \rightarrow T$ satisfying the conditions (11) and (2) of Theorem 5.2 in which $\pi_{1}^{\text {alg }}\left(X_{t}\right)$ is dominated by $\pi_{1}^{\text {alg }}$ of a $\mathbb{Q}$-Gorenstein smoothing of a geometric generic fiber of $X_{\Lambda} \rightarrow$ Spec $\Lambda$. Looking at the construction of $X_{\Lambda}$, we shall prove the simply connectedness of the $\mathbb{Q}$-Gorenstein smoothing of a geometric generic fiber from the argument used in the proof of [33, Theorem 3.1], [42, Theorem 3.1], and [43, Proposition 2.1]. The argument shows especially that, when $\mathbb{k}=\mathbb{C}, X \backslash \operatorname{Sing} X$ is topologically simply connected. In this way, the new $X_{t}$ is shown to be algebraically simply connected, and we have a desired surface.

Remark 6.1. In the construction above, the number $K^{2}$ must be between 1 and 4 . In fact, we may assume that $\operatorname{char}(\mathbb{k})=0$, and in this case, we have $\mathrm{H}^{0}\left(X_{t}, \Theta_{X_{t} / \mathbb{k}}\right)=0$, since the automorphism group of the surface $X_{t}$ of general type is finite. Thus, by Riemann-Roch,

$$
\operatorname{dim} \mathrm{H}^{1}\left(X_{t}, \Theta_{X_{t} / \mathbb{k}}\right)=-\chi\left(X_{t}, \Theta_{X_{t} / \mathbb{k}}\right)=10-2 K^{2},
$$

where we use $\chi\left(X_{t}, \mathcal{O}_{X_{t}}\right)=1, K_{X_{t}}^{2}=K^{2}$, and $\mathrm{H}^{2}\left(X_{t}, \Theta_{X_{t} / \mathbb{k}}\right)=0$. Hence, $1 \leq K^{2} \leq 5$. Moreover, our $X_{t}$ is a fiber of a $\mathbb{Q}$-Gorenstein smoothing of a rational surface with only cyclic quotient singularities of class $T$. Hence, $X_{t}$ has a non-trivial deformation by the existence of the coarse moduli of surfaces of general type (cf. [16, Theorem 1.3], [26, Corollary 5.7]). Thus, $\operatorname{dim} \mathrm{H}^{1}\left(X_{t}, \Theta_{X_{t} / \mathbb{k}}\right)>0$ and $K^{2} \leq 4$.

We shall explain the construction of $X$ and $X_{\Lambda} \rightarrow \operatorname{Spec} \Lambda$ from suitable cubic pencils on $\mathbb{P}^{2}$ step by step in Section 6. We also give sufficient conditions for the surface $X$ to be a desired one. Explicit examples of the cubic pencils are given in Section 7 , and the Main Theorem is proved using these examples.

Let us fix a complete discrete valuation ring $\Lambda$ of mixed characteristic with the residue field $\mathbb{k}$. Let $K^{2}$ be a given positive integer.

Step 1 . We first take two cubic homogeneous polynomials $\phi_{0}, \phi_{\infty}$ from $\mathbb{Z}[\mathrm{x}, \mathrm{y}, \mathrm{z}]$, and let $\Phi_{0}$ and $\Phi_{\infty}$ be the divisors of zeros of $\phi_{0}$ and $\phi_{\infty}$, respectively, on $\mathbb{P}_{\mathbb{k}}^{2}=\operatorname{Proj} \mathbb{k}[\mathrm{x}, \mathrm{y}, \mathrm{z}]$. Let $\Phi$ be the cubic pencil defined by $\Phi_{0}$ and $\Phi_{\infty}$. For $c \in \mathbb{k}$, let $\Phi_{c}$ be the divisor of zeros of $\phi_{0}+c \phi_{\infty}$. We here require the following conditions on $\Phi$ : 
$(\mathcal{C} 1)$ The base locus of $\Phi$, i.e. $\Phi_{0} \cap \Phi_{\infty}$, is a finite set.

$(\mathcal{C} 2)$ There exist at least two values of $c \in \mathbb{k} \backslash\{0\}$ such that $\Phi_{c}$ is singular. Moreover, if $\Phi_{c}$ is singular for $c \neq 0$, then it is a nodal rational curve.

Let $Y \rightarrow \mathbb{P}_{\mathbb{k}}^{2}$ be the elimination of the base locus of $\Phi$ which is a succession of blowings up at points. Then, we have a minimal elliptic fibration $\pi: Y \rightarrow \mathbb{P}_{\mathbb{k}}^{1}$ with a section such that $\mathcal{O}_{Y}\left(-K_{Y}\right) \simeq \pi^{*} \mathcal{O}(1)$.

Lemma 6.2. In the situation of Step 1 , there is a flat morphism $\pi_{\Lambda}: Y_{\Lambda} \rightarrow \mathbb{P}_{\Lambda}^{1}$ such that $\pi$ is the base change of $\pi_{\Lambda}$ by the closed immersion $\mathbb{P}_{\mathbb{k}}^{1} \rightarrow \mathbb{P}_{\Lambda}^{1}$. Let $c_{1}, c_{2}, \ldots, c_{n}$ be the values $c \in \mathbb{k}$ such that $\Phi_{c}$ is singular. Then, $c_{i}$ are regarded as elements of $\Lambda$, and hence $\pi_{\Lambda}$ is a smooth elliptic fibration outside the sections of $\mathbb{P}_{\Lambda}^{1} \rightarrow$ Spec $\Lambda$ defined by $(\mathrm{u}: \mathrm{v})=\left(1: c_{i}\right)$ for $1 \leq i \leq n$ and $(\mathrm{u}: \mathrm{v})=(0: 1)$, where $(\mathrm{u}: \mathrm{v})$ is a homogeneous coordinate of $\mathbb{P}^{1}$.

Proof. By construction, $\Phi_{0}$ and $\Phi_{\infty}$ are defined over $\mathbb{Z}$, and the each center of the blowing up between $Y$ and $\mathbb{P}_{\mathbb{k}}^{2}$ is a point whose coordinates are algebraic over $\mathbb{Z}$. Thus, the point is defined over the Henselian local ring $\Lambda$. Hence, $Y \rightarrow \mathbb{P}_{\mathbb{k}}^{2}$ extends to a birational morphism $Y_{\Lambda} \rightarrow \mathbb{P}_{\Lambda}^{2}$ which is a succession of blowings up along centers over Spec $\Lambda$. Moreover, by the pencil over $\Lambda$, we have an elliptic fibration $\pi_{\Lambda}: Y_{\Lambda} \rightarrow \mathbb{P}_{\Lambda}^{1}$ extending $\pi$. The elements $c_{1}, \ldots, c_{n}$ can be regarded as elements of $\Lambda$, since $\Lambda$ is Henselian, and $\pi_{\Lambda}$ is smooth outside the sections above.

Step 2. For the elliptic fibration $\pi$ in Step $\mathbb{1}$, after choosing two values $c_{1}, c_{2} \in \mathbb{k} \backslash\{0\}$ such that $\Phi_{c_{1}}$ and $\Phi_{c_{2}}$ are singular, we define $\bar{F}_{1}$ and $\bar{F}_{2}$ to be the proper transforms of $\Phi_{c_{1}}$ and $\Phi_{c_{2}}$ in $Y$, respectively. Note that, for $i=1,2, \bar{F}_{i}$ is the fiber over the point $\left(1: c_{i}\right) \in \mathbb{P}_{\mathbb{k}}^{1}$, and it is a singular fiber of type $\mathrm{I}_{1}$ in Kodaira's notation (cf. [25, Theorem 6.2]). We define $\bar{F}:=\bar{F}_{1}+\bar{F}_{2}$. Let $\bar{G}^{+}$be the union of all the $(-2)$-curves on $Y$. Note that every $(-2)$-curve on $Y$ is an irreducible component of a reducible fiber by $\mathcal{O}_{Y}\left(-K_{Y}\right) \simeq \pi^{*} \mathcal{O}(1)$, and thus it is an irreducible component of the total transform of $\Phi_{0}+\Phi_{\infty}$ in $Y$ (cf. (단 $)$ ). We choose $(-2)$-curves $\bar{G}_{1}, \bar{G}_{2}, \ldots, \bar{G}_{k}$ on $Y$ and set $\bar{G}:=\sum_{i=1}^{k} \bar{G}_{i}$. Moreover, we choose horizontal curves $\bar{S}_{1}, \bar{S}_{2}, \ldots, \bar{S}_{l}$ with respect to $\pi$ such that each $\bar{S}_{j}$ is either exceptional for $Y \rightarrow \mathbb{P}_{\mathbb{k}}^{2}$ or its image in $\mathbb{P}_{\mathbb{k}}^{2}$ is a line defined over the ring of algebraic integers. We set $\bar{S}:=\sum_{j=1}^{l} \bar{S}_{j}$, and also

$$
\bar{B}:=\bar{F}+\bar{G}+\bar{S} \quad \text { and } \quad \bar{B}^{+}:=\bar{F}+\bar{G}^{+}+\bar{S} .
$$

Note that, by construction and by Lemma 6.2, any irreducible component of $\bar{B}^{+}$is realized as the closed fiber of a prime divisor of $Y_{\Lambda}$ over $\operatorname{Spec} \Lambda$. We require the following conditions on $Y, \bar{B}^{+}$, and $\bar{G}$ : 
(C3) $\bar{B}^{+}$is a simple normal crossing divisor outside the nodes of $\bar{F}$.

$(\mathcal{C} 4) \mathcal{O}_{Y}\left(\bar{G}_{1}\right), \ldots, \mathcal{O}_{Y}\left(\bar{G}_{k}\right)$, and $\pi^{*} \mathcal{O}(1)$ are linearly independent in $\operatorname{Pic}(Y) \otimes_{\mathbb{Z}} \mathbb{k}$.

We do not require the following additional conditions $(\underline{\mathcal{A} 1})$ and $(\mathcal{A} 2)$ which are however useful to check the ampleness of $K_{X}$ (cf. Proposition 6.6, (41) below):

$(\mathcal{A} 1)$ The open set $Y \backslash(\bar{S} \cup \bar{G})$ is affine.

$(\mathcal{A} 2)$ The open set $Y \backslash(\bar{S} \cup \bar{G})$ does not contain any (-2)-curve.

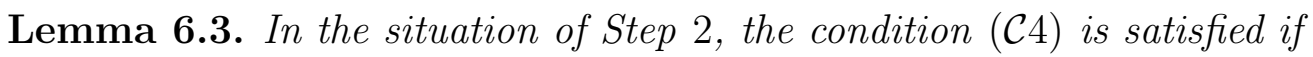

$$
\operatorname{det}\left(\bar{G}_{i} \bar{G}_{j}\right) \not \equiv 0 \quad \bmod \operatorname{char}(\mathbb{k}) .
$$

Proof. For $p:=\operatorname{char}(\mathbb{k})$, suppose that

$$
\sum_{i=1}^{k} c_{i} \bar{G}_{i}+c \bar{F}_{1} \sim p H
$$

for some integers $c_{i}$ and $c$, and for the fiber $\bar{F}_{1}$ of $\pi$ and a Cartier divisor $H$. Then, $c_{i} \equiv 0 \bmod p$ for any $1 \leq i \leq k$ by $\operatorname{det}\left(\bar{G}_{i} \bar{G}_{j}\right) \not \equiv 0 \bmod p$ and by $\bar{F}_{1} \bar{G}_{i}=0$. Moreover, $c \equiv 0 \bmod p$, since $\bar{F}_{1} \Sigma=1$ for a section $\Sigma$ of $\pi$. Thus, (난) is satisfied.

Lemma 6.4. In the situation of Step 2,

$$
\mathrm{H}^{0}\left(Y, \Omega_{Y / \mathbb{k}}^{1}\left(\log \left(\bar{G}+\bar{F}_{2}\right)\right)=0 .\right.
$$

Proof. Since $\bar{G}+\bar{F}_{2}$ is normal crossing (cf. (담) $)$, we can consider the exact sequence

$$
0 \rightarrow \Omega_{Y / \mathbb{k}}^{1} \rightarrow \Omega_{Y / \mathbb{k}}^{1}\left(\log \left(\bar{G}+\bar{F}_{2}\right)\right) \rightarrow \bigoplus_{i=1}^{k} \mathcal{O}_{\bar{G}_{i}} \oplus \mathcal{O}_{F_{2}} \rightarrow 0
$$

where $F_{2}$ is the normalization of $\bar{F}_{2}$. Let $f_{2} \in \mathrm{H}^{1}\left(Y, \Omega_{Y / \mathbb{k}}^{1}\right)$ be the image of $1 \in \mathrm{H}^{0}\left(\mathcal{O}_{F_{2}}\right)$ by the connecting homomorphism of the long exact sequence of cohomology groups associated with ( (VI-1). Similarly, let $g_{i} \in \mathrm{H}^{1}\left(Y, \Omega_{Y / \mathbb{k}}^{1}\right)$ be the image of $1 \in \mathrm{H}^{0}\left(\mathcal{O}_{G_{i}}\right)$ for $1 \leq i \leq k$. Then, $f_{2}$ and $g_{i}$ can be regarded as the first Chern classes (with respect to "dlog") of the invertible sheaves $\mathcal{O}_{Y}\left(\bar{F}_{2}\right)$ and $\mathcal{O}_{Y}\left(\bar{G}_{i}\right)$, respectively. In fact, we have a commutative diagram

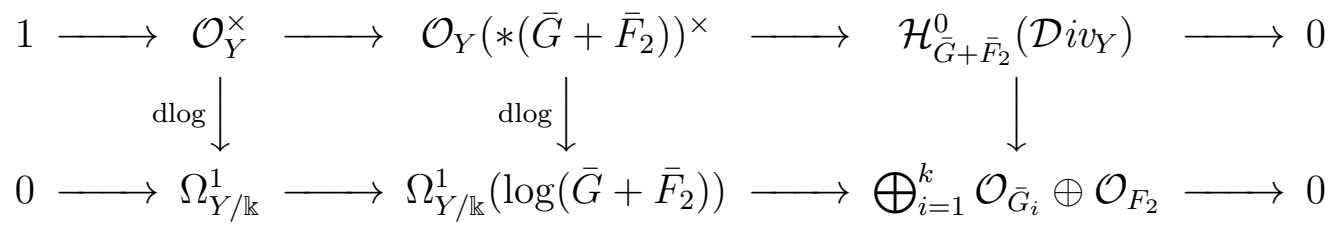

of exact sequence, where $\operatorname{dlog}(u)=u^{-1} \mathrm{~d} u$ for a rational function $u$, and for an effective divisor $R$,

- $\mathcal{O}_{Y}(* R)^{\times}$stands for the sheaf of invertible rational functions on $Y$ regular on the open subset $Y \backslash \operatorname{Supp} R$, and

- $\mathcal{H}_{R}^{0}\left(\mathcal{D} i v_{Y}\right)$ stands for the sheaf of Cartier divisors on $Y$ supported on Supp $R$. 
Note that, as global sections of $\mathcal{H}_{\bar{G}+\bar{F}_{2}}^{0}\left(\mathcal{D} i v_{Y}\right), \bar{F}_{2}$ and $\bar{G}_{i}$ are mapped to $1 \in \mathcal{O}_{F_{2}}$ and $1 \in \mathcal{O}_{\bar{G}_{i}}$, respectively, by the right vertical homomorphism. The first Chern class map $c: \operatorname{Pic}(Y)=\mathrm{H}^{1}\left(Y, \mathcal{O}_{Y}^{\times}\right) \rightarrow \mathrm{H}^{1}\left(Y, \Omega_{Y / \mathbb{k}}^{1}\right)$ is induced by the left vertical homomorphism: dlog. Therefore, $f_{2}=c\left(\mathcal{O}_{Y}\left(\bar{F}_{2}\right)\right)$ and $g_{i}=c\left(\mathcal{O}_{Y}\left(\bar{G}_{i}\right)\right)$. For the natural bilinear form

$$
\langle,\rangle: \mathrm{H}^{1}\left(Y, \Omega_{Y / \mathbb{k}}^{1}\right) \times \mathrm{H}^{1}\left(Y, \Omega_{Y / \mathbb{k}}^{1}\right) \rightarrow \mathrm{H}^{2}\left(Y, \Omega_{Y / \mathbb{k}}^{2}\right) \simeq \mathbb{k},
$$

the value $\left\langle c\left(\mathcal{L}_{1}\right), c\left(\mathcal{L}_{2}\right)\right\rangle$ in $\mathbb{k}$ equals the intersection number $\mathcal{L}_{1} \cdot \mathcal{L}_{2}$ modulo char $(\mathbb{k})$ for any invertible sheaves $\mathcal{L}_{1}, \mathcal{L}_{2}$ on $Y$ (cf. [18, Chapter. V, Exer. 1.8]). Now, $\operatorname{Pic}(Y)$ is a unimodular lattice for the intersection pairing, since $Y$ is rational. As a consequence, $c$ induces an injection $\operatorname{Pic}(Y) \otimes_{\mathbb{Z}} \mathbb{k} \rightarrow \mathrm{H}^{1}\left(Y, \Omega_{Y / \mathbb{k}}^{1}\right)$. Hence, $g_{1}, \ldots, g_{k}$, and $f_{2}$ are linearly independent by (다). Therefore, the connecting homomorphism for (VI-1) is injective, and we have

$$
\mathrm{H}^{0}\left(Y, \Omega_{Y / \mathbb{k}}^{1}\left(\log \left(\bar{G}+\bar{F}_{2}\right)\right)\right)=\mathrm{H}^{0}\left(Y, \Omega_{Y / \mathbb{k}}^{1}\right)=0 .
$$

Step 3. We consider the blowing up $\tau: Z \rightarrow Y$ at the two nodes $P_{1}$ and $P_{2}$ of $\bar{F}_{1}$ and $\bar{F}_{2}$, respectively. Let $F_{i}$ be the proper transform of $\bar{F}_{i}$ in $Z$ and let $J_{i}$ be the exceptional divisor $\tau^{-1}\left(P_{i}\right)$ for $i=1,2$. Then, $F_{i} \rightarrow \bar{F}_{i}$ is the normalization map, and $\tau^{*}\left(\bar{F}_{i}\right)=F_{i}+2 J_{i}$ for $i=1,2$. In particular,

$$
K_{Z} \sim-F_{1}-J_{1}+J_{2}, \quad \text { and } \quad-2 K_{Z} \sim F_{1}+F_{2} .
$$

Let $G_{i}$ and $S_{j}$ denote the proper transforms of $\bar{G}_{i}$ and $\bar{S}_{j}$ in $Z$ for $1 \leq i \leq k$ and $1 \leq j \leq l$. Here, $G_{i}$ is the total transform of $\bar{G}_{i}$, since $\bar{G}_{i} \cap \bar{F}=\emptyset$. We set $F:=F_{1}+F_{2}$, $G:=\sum_{i=1}^{k} G_{i}$, and $S:=\sum_{j=1}^{l} S_{j}$. We require the following conditions:

$(\mathcal{C} 5) S+F+J_{1}+J_{2}$ is a simple normal crossing divisor.

(C6) $S_{j}$ are $(-1)$-curves for any $1 \leq j \leq l$.

Let $B^{+}$be the total transform of $\bar{B}^{+}$in $Z$. Then, $B^{+}$is also a simple normal crossing

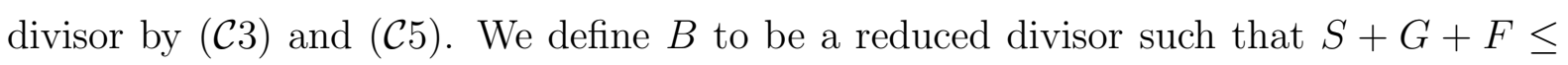
$B \leq S+G+F+J_{1}+J_{2}=B^{+}$.

Lemma 6.5. In the situation of Step 3,

$$
\mathrm{H}^{2}\left(Z, \Theta_{Z / \mathbb{k}}(-\log (G+F))\right)=\mathrm{H}^{2}\left(Z, \Theta_{Z / \mathbb{k}}(-\log B)\right)=0 .
$$

Proof. The second vanishing is derived from the first, since we have an exact sequence

$$
0 \rightarrow \Theta_{Z / \mathbb{k}}(-\log B) \rightarrow \Theta_{Z / \mathbb{k}}(-\log (G+F)) \rightarrow \bigoplus_{j=1}^{l} \mathcal{O}_{S_{j}}\left(S_{j}\right) \oplus \bigoplus_{J_{i} \subset B} \mathcal{O}_{J_{i}}\left(J_{i}\right) \rightarrow 0
$$

for the simple normal crossing divisors $B$ and $G+F$, in which $\mathrm{H}^{1}\left(\mathcal{O}_{S_{j}}\left(S_{j}\right)\right)=\mathrm{H}^{1}\left(\mathcal{O}_{J_{i}}\left(J_{i}\right)\right)$ $=\mathrm{H}^{1}\left(\mathbb{P}^{1}, \mathcal{O}(-1)\right)=0$ by $(\underline{\mathcal{C} 6})$. The first vanishing is equivalent to

$$
\mathrm{H}^{0}\left(Z, \Omega_{Z / \mathbb{k}}^{1}(\log (G+F)) \otimes \mathcal{O}_{Z}\left(K_{Z}\right)\right)=0
$$


by Serre duality. By ( $(\overline{V I}-2)$, we have an inclusion

$$
\begin{aligned}
\tau_{*}\left(\Omega_{Z / \mathbb{k}}^{1}(\log (G+F)) \otimes \mathcal{O}_{Z}\left(K_{Z}\right)\right) & \hookrightarrow \tau_{*}\left(\Omega_{Z / \mathbb{k}}^{1}\left(\log \left(G+F_{2}\right)\right) \otimes \mathcal{O}_{Z}\left(J_{2}\right)\right) \\
& \hookrightarrow \Omega_{Y / \mathbb{k}}^{1}\left(\log \left(\bar{G}+\bar{F}_{2}\right)\right),
\end{aligned}
$$

where the last map is the injection to the double-dual. Thus, we are done by Lemma 6.4.

Step 4. We take a successive blowings up $\varphi: M \rightarrow Z$ whose centers are certain nodes of the total transform of $B^{+}$. On the choice of nodes, we assume that the total transform $B_{M}=\varphi^{-1}(B)$ of $B$ in $M$ contains a disjoint union $D=\bigcup_{i=1}^{m} D^{(i)}$ of linear chains $D^{(1)}$, $\ldots, D^{(m)}$ of smooth rational curves satisfying the following conditions $(\underline{\mathcal{C} 7})-(\underline{\mathcal{C} 11})$ :

(C7) $\varphi(D)=B$.

$(\mathcal{C} 8)$ Each $D^{(i)}$ contains no $(-1)$-curve, and is contractible to a toric singularity of class $\mathrm{T}$.

Let $\mu: M \rightarrow X$ be the contraction morphism of $D$. Then, $X$ has only toric singularities of class $\mathrm{T}$ and $\mu$ is the minimal resolution of singularities. Let $\Delta$ be the $\mathbb{Q}$-divisor supported on $D$ define by $K_{M}+\Delta=\mu^{*}\left(K_{X}\right)$.

(C9) $K_{X}^{2}=K^{2}$

$(\mathcal{C} 10) \Delta \Gamma>1$ for any $\varphi$-exceptional $(-1)$-curve $\Gamma$ on $M$.

$(\mathcal{C} 11)$ For any $D^{(i)}$, there exist smooth rational curves $\Gamma$ and $\Gamma^{\prime}$ not contained in $D^{(i)}$ such that

- $\Gamma+D^{(i)}+\Gamma^{\prime}$ is a linear chain of smooth rational curves with the end components $\Gamma$ and $\Gamma^{\prime}$,

- $\varphi\left(\Gamma \cup \Gamma^{\prime}\right)$ is contained in $B^{+}$.

We do not require the following condition ( $(\underline{A} 3)$ which is however useful for checking the ampleness of $K_{X}$ (cf. Proposition 6.6, (44) below):

$(\mathcal{A} 3)$ There is no $\varphi$-exceptional $(-2)$-curve contained in $M \backslash D$.

Proposition 6.6. In the situation of Step 4, the following hold:

(1) $\mathrm{H}^{1}\left(X, \mathcal{O}_{X}\right)=\mathrm{H}^{2}\left(X, \mathcal{O}_{X}\right)=0$.

(2) $\mathrm{H}^{2}\left(M, \Theta_{M / \mathbb{k}}(-\log D)\right)=\mathrm{H}^{2}\left(X, \Theta_{X}\right)=0$.

(3) $K_{X}$ is nef and big, and it is $\mathbb{Q}$-linearly equivalent to an effective $\mathbb{Q}$-divisor whose support is the union of $D$ and the $\varphi$-exceptional locus.

(4) $K_{X}$ is ample if and only if there is no $(-2)$-curve contained in $M \backslash D$. If (A1) and (A3) are satisfied, then $K_{X}$ is ample. If $K_{X}$ is ample, then (A2) and (A3) are satisfied. In case $B$ contains $J_{1}$ or $J_{2}, K_{X}$ is ample if and only if (A2) and (A3) are satisfied. 
Proof. (1) follows from that $X$ has only rational singularities and $M$ is rational.

(2): By Corollary 2.12, it suffices to prove $\mathrm{H}^{2}\left(M, \Theta_{M / \mathbb{k}}(-\log D)\right)=0$. Now $B_{M}=$ $\varphi^{-1}(B)$ is a simple normal crossing divisor containing $D$. Since the cokernel of the natural injection $\Theta_{M / \mathbb{k}}\left(-\log B_{M}\right) \hookrightarrow \Theta_{M / \mathbb{k}}(-\log D)$ is supported on the one-dimensional subscheme $B_{M}-D$, it is enough to prove: $\mathrm{H}^{2}\left(M, \Theta_{M / \mathbb{k}}\left(-\log B_{M}\right)\right)=0$. We have an isomorphism $\Theta_{M / \mathbb{k}}\left(-\log B_{M}\right) \simeq \varphi^{*} \Theta_{Z / \mathbb{k}}(-\log B)$, since the center of the each step of the successive blowings up $\varphi$ is a node of the total transform of $B$. Hence, by Lemma 6.5, we have

$$
\mathrm{H}^{2}\left(M, \Theta_{M / \mathbb{k}}\left(-\log B_{M}\right)\right) \simeq \mathrm{H}^{2}\left(Z, \Theta_{Z / \mathbb{k}}(-\log B)\right)=0 .
$$

(3): By (VI-2), $K_{Z}+(1 / 2)\left(F_{1}+F_{2}\right) \sim_{\mathbb{Q}} 0$. Since $F_{1}+F_{2}$ is smooth, the pair $\left(Z,(1 / 2)\left(F_{1}+F_{2}\right)\right)$ is terminal (in the sense of [27, Definition 1.16]). Hence, $K_{M}+$ $(1 / 2)\left(F_{1}^{\prime}+F_{2}^{\prime}\right) \sim_{\mathbb{Q}} A$ for an effective $\mathbb{Q}$-divisor $A$ whose support is just the exceptional locus for $\varphi: M \rightarrow Z$. Thus,

$$
K_{M}+\Delta \sim_{\mathbb{Q}} A+\Delta-(1 / 2)\left(F_{1}^{\prime}+F_{2}^{\prime}\right) .
$$

Here, the right hand side is an effective $\mathbb{Q}$-divisor whose support is the union of $D$ and the $\varphi$-exceptional locus, since

$$
\operatorname{mult}_{F_{i}^{\prime}}(\Delta)>1 / 2 \quad \text { for } \quad i=1,2,
$$

by $F_{i}^{\prime 2} \leq F_{i}^{2}=-4$ and by Corollary 3.7.2 and Lemma 3.6. If $K_{M}+\Delta$ is nef, then this is also big by (다) . Thus, it suffices to derive a contradiction assuming that $K_{M}+\Delta$ is not nef. Then, there is a curve $\Gamma$ with $\left(K_{M}+\Delta\right) \Gamma<0$. By (VI-3),$\Gamma$ is $\varphi$-exceptional or $\mu$-exceptional. But $\Gamma$ is not $\mu$-exceptional; for otherwise, $\left(K_{M}+\Delta\right) \Gamma=\mu^{*}\left(K_{X}\right) \Gamma=0$. Thus, $\Gamma$ is $\varphi$-exceptional. Hence, $\Delta \Gamma \geq 0$ and $K_{M} \Gamma<0$; consequently, $\Gamma$ is a $(-1)$-curve

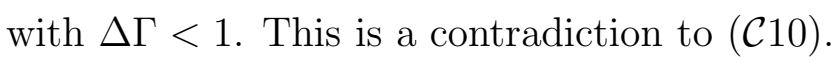

(44): By Nakai-Moishezon's criterion, $K_{X}$ is not ample if and only if there is a curve $\Gamma$ such that it is not $\mu$-exceptional and $\left(K_{M}+\Delta\right) \Gamma=0$. Let $\Gamma$ be such a curve. Then,

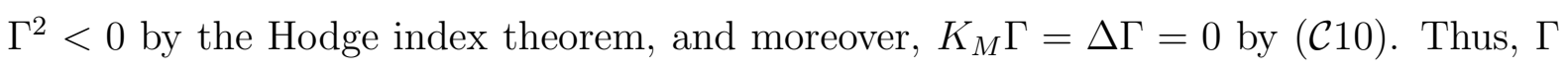
is a (-2)-curve contained in $M \backslash D$. Conversely, if $\Gamma$ is a $(-2)$-curve in $M \backslash D$, then $K_{X}$ is not ample by $K_{X} \varphi_{*}(\Gamma)=\left(K_{M}+\Delta\right) \Gamma=0$.

Assume that the $(-2)$-curve $\Gamma$ above is not $\varphi$-exceptional. Then, $\Gamma$ does not intersect the $\varphi$-exceptional locus, since $A \Gamma=0$ by ( $(\overline{V I-3})$. Hence, $\varphi(\Gamma) \cap \varphi(D)=\emptyset$. In particular,

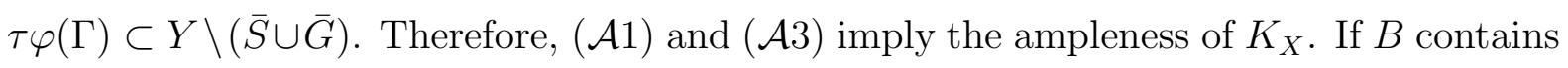
$J_{1}$, then $\varphi(\Gamma)$ does not intersect the fiber $F_{1} \cup J_{1}$; hence, $\tau \varphi(\Gamma)$ is a $(-2)$-curve contained

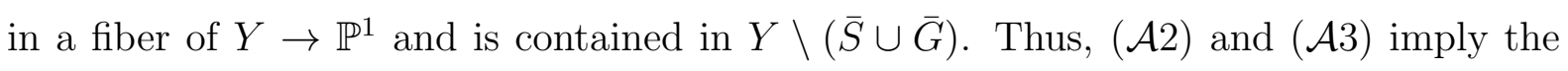
ampleness of $K_{X}$ in this case. If (슬 or $(\underline{\mathcal{A} 3})$ fails, then we can find an irreducible curve $\Gamma$ contained in $M \backslash D$; thus $K_{X}$ is not ample. 
Remark. Our proof of Proposition 6.6.(2) is different from the proof of the corresponding result in [33, Section 4], but has many common ideas.

Lemma 6.7. In Step 4, the condition (든 is derived from the following three conditions for all the $\varphi$-exceptional $(-1)$-curves $\Gamma$ :

(1) $\Gamma$ intersects at least two irreducible components of $D$.

(2) If $\Gamma$ intersects a connected component of $D$ which defines a toric singularity of type $T(l, 2,1)$ for some $l \geq 1$, then $\Gamma$ intersects another component of $D$ defining a toric singularity of type $T(d, n, a)$ with $n>2$.

(3) $\Delta \Gamma>1$ if $\Gamma$ intersects a (-2)-curve belonging to a sequence of $(-2)$-curves at an end of a connected component of $D$.

Proof. Let $\Gamma$ be a $\varphi$-exceptional $(-1)$-curve. Assume that $\Gamma$ intersects two irreducible components $\Xi_{1}, \Xi_{2}$ of $D$ such that

- $\Xi_{i}$ does not belong to any sequence of $(-2)$-curves at an end of a connected component of $D$ for $i=1,2$,

- the linear chain containing $\Xi_{1}$ defines a singularity of type $T(d, n, a)$ with $n>1$. It suffices to prove $\Delta \Gamma>1$. By Lemma 3.7.2, the multiplicity of $\Delta$ along $\Xi_{1}$ is greater than $1 / 2$ and the multiplicity along $\Xi_{2}$ is greater than or equal to $1 / 2$. Thus, we have $\Delta \Gamma>1$.

Applying Theorem 5.2 to $X$ above, we have:

Proposition 6.8. Let $\Phi$ be a cubic pencil on $\mathbb{P}_{\mathbb{k}}^{2}$ satisfying $(\underline{\mathcal{C} 1})$ and $(\underline{\mathcal{C} 2})$, and let $Y \rightarrow \mathbb{P}^{2}$ be the elimination of the base locus of $\Phi$ by a succession of blowings up at points. Let $\bar{F}$,

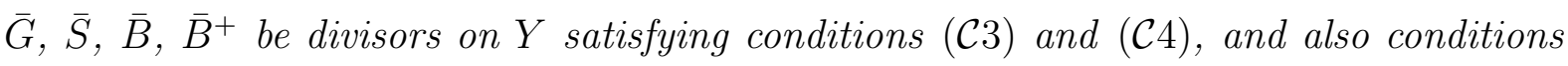
$(\underline{\mathcal{C} 5})$ and (다) on the blown up surface $Z$ at the nodes of $\bar{F}$. Let $\varphi: M \rightarrow Z$ be a succession of blowings up at certain nodes of the total transform of $\bar{B}^{+}$, and let $D=\sum D^{(i)}$ be a disjoint union of linear chains of smooth rational curves contained in the total transform of $\bar{B}$ in which the conditions (드)-(데) are satisfied. Then, there is a minimal projective surface $\mathbb{S}$ of general type defined over $\mathbb{k}$ such that $p_{g}(\mathbb{S})=q(\mathbb{S})=0, K_{\mathbb{S}}^{2}$ equals the given integer $1 \leq K^{2} \leq 4$ in the Main Theorem, and $\mathrm{H}^{2}\left(\mathbb{S}, \Theta_{\mathbb{S} / \mathbb{k}}\right)=0$. If $M \backslash D$ contains no $(-2)$-curves, we can even assume the canonical divisor $K_{\mathbb{S}}$ to be ample.

Step 5. Let $B_{M}^{+}$be the total transform of $B^{+}$in $M$. This is a simple normal crossing divisor consisting of rational curves. Since $\tau \circ \varphi: M \rightarrow Y$ is a succession of blowings up whose centers are nodes of the total transform of $\bar{B}$, by Lemma 6.2 and by the condition on $\bar{S}_{j}$ in Step 2, we can realize $M$ as the closed fiber of a smooth projective family $M_{\Lambda} \rightarrow \operatorname{Spec} \Lambda$ of rational surfaces and $B_{M}^{+}$as the closed fiber of a divisor $B_{M_{\Lambda}}^{+}$on $M_{\Lambda}$ flat 
over $\Lambda$ such that every irreducible component of $B_{M_{\Lambda}}^{+}$is a $\mathbb{P}^{1}$-bundle over $\Lambda$ and that any non-empty intersection of two prime divisors of $B_{M_{\Lambda}}^{+}$is a section over Spec $\Lambda$. Moreover, we have a relative Cartier divisor on $M_{\Lambda}$ whose restriction to $M$ is linearly equivalent to the pullback of an ample divisor on $X$. For, the exceptional divisors for the birational morphism $M_{\Lambda} \rightarrow Y_{\Lambda} \rightarrow \mathbb{P}_{\Lambda}^{2}$ and the pullback of $\mathcal{O}_{\mathbb{P}^{2}}(1)$ generate $\operatorname{Pic}\left(M_{\Lambda}\right)$. Then, by Proposition 6.9 below, we have a projective family $X_{\Lambda} \rightarrow \operatorname{Spec} \Lambda$ of normal projective surfaces and a birational morphism $\mu_{\Lambda}: M_{\Lambda} \rightarrow X_{\Lambda}$ over Spec $\Lambda$ such that the closed fiber of $X_{\Lambda}$ is isomorphic to $X$ and the morphism $\mu_{\Lambda}$ restricted to the closed fibers is just the minimal resolution $\mu: M \rightarrow X$.

Proposition 6.9. Let $\Lambda$ be a discrete valuation ring with the residue field $\mathbb{k}$ which is algebraically closed. Let $V$ be a normal projective surface with only rational singularities defined over $\mathbb{k}$. Let $\widetilde{W} \rightarrow \operatorname{Spec} \Lambda$ be a smooth projective morphism such that $W:=$ $\widetilde{W} \times{ }_{\operatorname{Spec} \Lambda} \operatorname{Spec} \mathbb{k}$ is the minimal resolution of singularities of $V$. Let $\left\{\widetilde{E}_{i}\right\}_{i \in I}$ be a set of prime divisors on $\widetilde{W}$ such that:

- $\widetilde{E}_{i}$ is a $\mathbb{P}^{1}$-bundle over $\operatorname{Spec} \Lambda$ for any $i \in I$.

- $E_{i}=\widetilde{E}_{i} \times_{\operatorname{Spec} \Lambda} \operatorname{Spec} \mathbb{k}$ is an irreducible component of the exceptional locus $E$ of the minimal resolution $\nu: W \rightarrow V$.

- Conversely, any irreducible component of $E$ is equal to $E_{i}$ for a unique $i \in I$.

- $\widetilde{E}_{i} \cap \widetilde{E}_{j}$ is a section of $\widetilde{W} \rightarrow \operatorname{Spec} \Lambda$ if $E_{i} \cap E_{j} \neq \emptyset$.

Assume that there is a divisor $\widetilde{L}$ on $\widetilde{W}$ such that $L:=\left.\widetilde{L}\right|_{W}$ is linearly equivalent to the pullback of an ample divisor on $V$. Then, there exist a normal projective $\Lambda$-scheme $\widetilde{V}$ and a proper birational morphism $\tilde{\nu}: \widetilde{W} \rightarrow \widetilde{V}$ satisfying the following conditions:

(1) $V \simeq \widetilde{V} \times_{\operatorname{Spec} \Lambda} \operatorname{Spec} \mathbb{k}$, and $\nu$ is obtained by the base change of $\widetilde{\nu}$ by Spec $\mathbb{k} \rightarrow$ $\operatorname{Spec} \Lambda$.

(2) The $\tilde{\nu}$-exceptional locus is $\bigcup \widetilde{E}_{i}$, and $\tilde{\nu}\left(\widetilde{E}_{i}\right)$ is a section of $\widetilde{V} \rightarrow \operatorname{Spec} \Lambda$ for all $i \in I$,

(3) If $r K_{V}$ is Cartier for a positive integer $r$, then so is $r K_{\widetilde{V}}$ and $\left.r K_{\widetilde{V}}\right|_{V} \sim r K_{V}$.

Proof. We may assume that $L=\nu^{*}\left(L_{V}\right)$ for a very ample divisor $L_{V}$ on $V$ such that $\mathrm{H}^{i}\left(V, \mathcal{O}_{V}\left(L_{V}\right)\right)=0$ for all $i>0$, by replacing $L$ with $m L$ for $m \gg 0$. Since $V$ has only rational singularities, $\mathrm{H}^{i}\left(W, \mathcal{O}_{W}(L)\right)=0$ for any $i>0$. The morphism defined by the base point free linear system $|L|$ is just $\nu: W \rightarrow V$ followed by a closed immersion $V \hookrightarrow \mathbb{P}^{N}$, where $N=\operatorname{dim}|L|$. We can show:

Claim 6.9.1. $\quad$ (i) $\mathrm{H}^{i}\left(\widetilde{W}, \mathcal{O}_{\widetilde{W}}(\widetilde{L})\right)=0$ for any $i>0$.

(ii) The natural homomorphism $\mathrm{H}^{0}\left(\widetilde{W}, \mathcal{O}_{\widetilde{W}}(\widetilde{L})\right) \otimes_{\Lambda} \mathbb{k} \rightarrow \mathrm{H}^{0}\left(W, \mathcal{O}_{W}(L)\right)$ is an isomorphism. 
(iii) $\mathcal{O}_{\widetilde{W}}(\widetilde{L})$ is generated by global sections, i.e., $\mathrm{H}^{0}\left(\widetilde{W}, \mathcal{O}_{\widetilde{W}}(\widetilde{L})\right) \otimes_{\Lambda} \mathcal{O}_{\widetilde{W}} \rightarrow \mathcal{O}_{\widetilde{W}}(\widetilde{L})$ is surjective.

Proof. In fact, (ii) is a consequence of the upper semi-continuity theorem for $\widetilde{W} \rightarrow \operatorname{Spec} \Lambda$ and the vanishing $\mathrm{H}^{i}\left(W, \mathcal{O}_{W}(L)\right)=0$ for any $i>0$. Then, (iii) is obtained as the base change isomorphism. The homomorphism in (iii) is surjective after tensoring $\mathbb{k}$ over $\Lambda$, by the freeness of $|L|$ and by (iii). Thus, the homomorphism is surjective along the closed fiber of $\widetilde{W} \rightarrow \operatorname{Spec} \Lambda$, and is surjective everywhere on $\widetilde{W}$, since $\Lambda$ is a local ring.

We continue the proof of Proposition 6.9, Now, by Claim 6.9.1.(iii) above, we see that $\mathrm{H}^{0}\left(\widetilde{W}, \mathcal{O}_{\widetilde{W}}(\widetilde{L})\right)$ is a free $\Lambda$-module of rank $N$, and the surjection in Claim 6.9.1.(iii) defines a morphism $\widetilde{W} \rightarrow \mathbb{P}_{\Lambda}^{N}$. Let $\widetilde{W} \rightarrow \widetilde{V} \rightarrow \mathbb{P}_{\Lambda}^{N}$ be the Stein factorization of $\widetilde{W} \rightarrow \mathbb{P}_{\Lambda}^{N}$. Then, $\widetilde{V}$ is normal and the fiber over Spec $\mathbb{k}$ of the proper morphism $\tilde{\nu}: \widetilde{W} \rightarrow \widetilde{V}$ is just $\nu: W \rightarrow V$.

By construction, $\tilde{\nu}$ is an isomorphism on $W \backslash \bigcup E_{i}$; hence $\tilde{\nu}$ is a birational morphism. Every $\widetilde{E}_{i}$ for $i \in I$ is $\tilde{\nu}$-exceptional, since $\left.\mathcal{O}_{\widetilde{W}}(\widetilde{L})\right|_{\widetilde{E}_{i}} \simeq \mathcal{O}_{\widetilde{E}_{i}}$. We shall show that there is no other $\tilde{\nu}$-exceptional divisor. Let $\widetilde{\Gamma}$ be a $\tilde{\nu}$-exceptional prime divisor on $\widetilde{W}$. If $\widetilde{\Gamma}$ is not flat over $\Lambda$, then $\widetilde{\Gamma}$ is contained in the closed fiber $W$ and also contained in some $E_{i}$; this is a contradiction, $\operatorname{since} \operatorname{codim}\left(E_{i}, \widetilde{W}\right)=2$. Thus, $\widetilde{\Gamma}$ is flat over $\Lambda$. Then, the closed fiber $\Gamma$ of $\widetilde{\Gamma} \rightarrow \operatorname{Spec} \Lambda$ is a union of $\nu$-exceptional curves. Here, the intersection number $b:=\Gamma E_{i}$ is negative for some $i \in I$. Hence, $\widetilde{\Gamma}=\widetilde{E}_{i}$, since $\left.\mathcal{O}_{\widetilde{W}}(\widetilde{\Gamma})\right|_{\widetilde{E}_{i}}$ is isomorphic to $\mathcal{O}_{\mathbb{P}_{\Lambda}^{1}}(b)$. Therefore, the $\tilde{\nu}$-exceptional locus is $\bigcup \widetilde{E}_{i}$. Since $\widetilde{E}_{i}$ is a $\mathbb{P}^{1}$-bundle over $\Lambda$, the image $\tilde{\nu}\left(\widetilde{E}_{i}\right)$ is a section of $\widetilde{V} \rightarrow \operatorname{Spec} \Lambda$. Thus, all the assertions except (3) are satisfied.

For (3), it suffices to show that $r K_{\widetilde{V}}$ is Cartier. In fact, if $r K_{\widetilde{V}}$ is Cartier, then $\left.r K_{\widetilde{V}}\right|_{V} \sim$ $r K_{V}$ by Remark 3.9.3. We consider the rational numbers $b_{i} \geq 0$ such that $\nu^{*}\left(K_{X}\right)=$ $K_{W}+\sum b_{i} E_{i}$. Then, $r b_{i} \in \mathbb{Z}$ for any $i$. Let $\widetilde{D}$ be the Cartier divisor $r K_{\widetilde{W}}+\sum r b_{i} \widetilde{E}_{i}$ on $\widetilde{W}$. Then, $\left.\widetilde{D}\right|_{W} \sim \nu^{*}\left(r K_{V}\right)$. We apply the argument above to $\widetilde{L}^{\prime}=a \widetilde{L}+\widetilde{D}$ for $a \gg 0$ instead of $\widetilde{L}$, where $a L_{V}+r K_{V}$ is ample on $V$. Then, $\mathcal{O}_{\widetilde{W}}\left(\widetilde{L^{\prime}}\right)$ is generated by global sections and defines a morphism $\widetilde{W} \rightarrow \mathbb{P}_{\Lambda}^{N^{\prime}}$ for some $N^{\prime}>0$. Let $\widetilde{\nu}^{\prime}: \widetilde{W} \rightarrow \widetilde{V^{\prime}}$ be the birational morphism obtained as the Stein factorization. Then, the exceptional locus of $\widetilde{W} \rightarrow \widetilde{V^{\prime}}$ is just the union of $\bigcup \widetilde{E}_{i}$. By Zariski's main theorem, we have an isomorphism $\widetilde{V} \simeq \widetilde{V^{\prime}}$ compatible with $\tilde{\nu}$ and $\tilde{\nu}^{\prime}$. Consequently, $\widetilde{D}$ is the pullback of a Cartier divisor on $\widetilde{V}$. Hence $r K_{\widetilde{V}}$ is Cartier and $\widetilde{D}=\tilde{\nu}^{*}\left(r K_{\widetilde{V}}\right)$ by $\tilde{\nu}_{*}(\widetilde{D})=\tilde{\nu}_{*}\left(r K_{\widetilde{W}}\right)=r K_{\widetilde{V}}$. Thus, we have finished the proof of Proposition 6.9.

Step 6. We can apply Theorem 5.4 to $X_{\Lambda} \rightarrow \operatorname{Spec} \Lambda$ constructed in Step 5 . In fact, the assumptions (ii) and (iii) of Theorem 5.4 are confirmed by Proposition 6.6 and by an argument in Step 4 and the condition (데), respectively. Let $X_{\mathbb{K}}$ be the geometric 
generic fiber of $X_{\Lambda} \rightarrow \operatorname{Spec} \Lambda$ for an algebraically closed field $\mathbb{K}$ containing $\Lambda$. Thus, by Theorem 5.4 and by Corollary 5.5, we have deformations $\mathcal{X} \rightarrow T$ of $X$ and $\mathcal{X}_{\mathbb{K}} \rightarrow T_{\mathbb{K}}$ of $\bar{X}$ such that:

- $T$ is a non-singular algebraic curve over $\mathbb{k}$, and $\mathcal{X} \rightarrow T$ satisfies the conditions (1) and (2) of Theorem 5.2.

- $T_{\mathbb{K}}$ is a non-singular algebraic curve over $\mathbb{K}$, and $\mathcal{X}_{\mathbb{K}} \rightarrow T_{\mathbb{K}}$ satisfies the conditions (11) and (2) of Theorem 5.2.

- $\pi_{1}^{\text {alg }}\left(X_{\mathbb{K}, t^{\prime}}\right) \rightarrow \pi_{1}^{\text {alg }}\left(X_{t}\right)$ is surjective for any closed smooth fibers $X_{t}=\mathcal{X} \times_{T} t$ and $X_{\mathbb{K}, t^{\prime}}=\mathcal{X}_{\mathbb{K}} \times_{T_{\mathbb{K}}} t^{\prime}$

In order to get the algebraic simply connectedness of $X_{\mathbb{K}, t^{\prime}}$, we shall compare $X_{\mathbb{K}}$ and a normal projective surface $\bar{X}$ defined over $\mathbb{C}$ which is constructed by the same method as in Steps 14 .

Let $Y_{\mathbb{C}} \rightarrow \mathbb{P}_{\mathbb{C}}^{1}$ be the elliptic fibration obtained from the pencil $\Phi$ on $\mathbb{P}_{\mathbb{C}}^{2}$ by replacing $\mathbb{k}$ with $\mathbb{C}$. Then, by the same process as in Steps 3 and 4 , we have birational morphisms $\tau_{\mathbb{C}}: Z_{\mathbb{C}} \rightarrow Y_{\mathbb{C}}, \varphi_{\mathbb{C}}: M_{\mathbb{C}} \rightarrow Z_{\mathbb{C}}$, and a disjoint union $D_{\mathbb{C}}$ of linear chains of smooth rational curves such that the contraction of $D_{\mathbb{C}}$ is the minimal resolution $\mu_{\mathbb{C}}: M_{\mathbb{C}} \rightarrow X_{\mathbb{C}}$ of a normal projective surface $X_{\mathbb{C}}$ with only toric singularities of class $\mathrm{T}$. Let $\mathbb{K}_{0}$ be a subfield of $\mathbb{K}$ finitely generated over $\mathbb{Q}$ such that $X_{\mathbb{K}}$, Sing $X_{\mathbb{K}}$, and $\bar{X} \rightarrow \bar{T}$ are defined over $\mathbb{K}_{0}$. We take an injection $\mathbb{K}_{0} \hookrightarrow \mathbb{C}$ and set $\bar{X}:=X_{\mathbb{K}_{0}} \times_{\text {Spec } \mathbb{K}_{0}}$ Spec $\mathbb{C}$. Then, $\bar{X} \simeq X_{\mathbb{C}}$ by our construction of $X_{\Lambda} \rightarrow \operatorname{Spec} \Lambda$.

Lemma 6.10. If $X_{\mathbb{C}} \backslash \operatorname{Sing} X_{\mathbb{C}} \simeq M_{\mathbb{C}} \backslash D_{\mathbb{C}}$ is simply connected (with respect to the Euclidean topology), then a general smooth closed fiber $X_{t}$ of $\mathcal{X} \rightarrow T$ is algebraically simply connected.

Proof. By the assumption, any $\mathbb{Q}$-Gorenstein smoothing of $X_{\mathbb{C}}$ is simply connected by an argument in the proof of [33, Theorem 3.1] using results on rational blowdown 4manifolds, Milnor fibers, and van-Kampen's theorem. Thus, by Remark 5.5.1, a smooth closed fiber $X_{\mathbb{K}, t^{\prime}}$ of $\mathcal{X}_{\mathbb{K}} \rightarrow T_{\mathbb{K}}$ is algebraically simply connected. Therefore, so is $X_{t}$ by Corollary 5.5 .

The following is useful for proving the simply connectedness of $M_{\mathbb{C}} \backslash D_{\mathbb{C}}$ and used in the proof of [33, Theorem 3.1].

Lemma 6.10.1. In the construction of $M$ and $D$ above, assume that $\mathbb{k}=\mathbb{C}$. Then, $M \backslash D$ is simply connected (with respect to the euclidean topology) provided that, for any connected component $D_{i}$ of $D$, there exists a smooth rational curve $E$ on $M$ and another connected component $D_{j}$ of $D$ such that:

(1) E intersects an end component of $D_{i}$ and an end component of $D_{j}$. 
(2) $E D_{i}=E D_{j}=1$, and $E$ does not intersect the other connected components of $D$.

(3) $\operatorname{gcd}\left(d_{i} n_{i}, d_{j} n_{j}\right)=1$ for the types $T\left(d_{i}, n_{i}, a_{i}\right)$ and $T\left(d_{j}, n_{j}, a_{j}\right)$ of the toric singularities defined by $D_{i}$ and $D_{j}$, respectively.

Proof. Let $Q_{i}$ be the toric singular point $\mu\left(D_{i}\right)$ of class $\mathrm{T}$ and let $T\left(d_{i}, n_{i}, a_{i}\right)$ be the type. We have an open neighborhood $\overline{\mathcal{U}}_{i}$ (with respect to the euclidean topology) of $Q_{i}$ and a finite surjective morphism $\lambda_{i}: \mathcal{U}_{i}^{\sim} \rightarrow \overline{\mathcal{U}}_{i}$ such that

- $\overline{\mathcal{U}}_{i} \cap \operatorname{Sing} X=\left\{Q_{i}\right\}$,

- $\overline{\mathcal{U}}_{i}$ is topologically contractible,

- $\mathcal{U}_{i}^{\sim}$ is a non-singular surface topologically contractible to a point,

- $\lambda_{i}$ is étale over $\overline{\mathcal{U}}_{i} \backslash\left\{Q_{i}\right\}$,

- $\lambda_{i}$ is a cyclic covering of degree $d_{i} n_{i}^{2}$.

In particular, $\pi_{1}\left(\overline{\mathcal{U}}_{i} \backslash\left\{Q_{i}\right\}\right)$ is a cyclic group of order $d_{i} n_{i}^{2}$. We set $\mathcal{U}_{i}:=\mu^{-1}\left(\overline{\mathcal{U}}_{i}\right)$, which is homotopic to $D_{i}$. Hence, $\mathcal{U}_{i}$ is simply connected. Moreover, $\mathcal{U}_{i} \backslash D=\mathcal{U}_{i} \backslash D_{i} \simeq \overline{\mathcal{U}}_{i} \backslash Q_{i}$. We may assume that $\mathcal{U}_{i} \cap \mathcal{U}_{j}=\emptyset$ for any $i, j$. For the open immersion $\mathcal{U}_{i} \backslash D \hookrightarrow M \backslash D$, we have a homomorphism $\pi_{1}\left(\mathcal{U}_{i} \backslash D\right) \rightarrow \pi_{1}(M \backslash D)$ defined up to conjugate by considering a path connecting the reference points of $\mathcal{U}_{i} \backslash D$ and $M \backslash D$. Let $\gamma_{i}$ be the image of a generator of $\pi_{1}\left(\mathcal{U}_{i} \backslash D\right)$ in $\pi_{1}(M \backslash D)$. Then, $\pi_{1}(M \backslash D)$ is generated by the images $\gamma_{i}$ for any $i$ by van-Kampen's theorem applied to the open covering $M=(M \backslash D) \cup \cup \mathcal{U}_{i}$, since $M$ and $\mathcal{U}_{i}$ are simply connected.

For a connected component $D_{i}$, let $D_{j}$ be another connected component and $E$ be a smooth rational curve satisfying (11)-(3). Then, $E \backslash D \simeq \mathbb{C} \backslash\{0\}$. Let $\gamma_{E}$ be the image of a generator of the cyclic group $\pi_{1}(E \backslash D)$ in $\pi_{1}(M \backslash D)$ by a homomorphism $\pi_{1}(E \backslash D) \rightarrow \pi_{1}(M \backslash D)$ defined up to conjugate similarly to the above from the inclusion $E \backslash D \hookrightarrow M \backslash D$. Then, $\gamma_{E}$ is conjugate to $\gamma_{i}$ or $\gamma_{i}^{-1}$, and conjugate to $\gamma_{j}$ or $\gamma_{j}^{-1}$, since $E$ intersects $D_{i}$ (resp. $D_{j}$ ) transversely only at one point which is in an end component. Therefore, $\gamma_{i}$ is conjugate to $\gamma_{j}$ or $\gamma_{j}^{-1}$. Hence, $\gamma_{i}=\gamma_{j}=1$ by (3). This proves that $M \backslash D$ is simply connected.

By the discussion in Steps [5] and [6, we have:

Proposition 6.11. In the situation of Proposition 6.8, let $M_{\mathbb{C}}$ be the same surface obtained as above by replacing $\mathbb{k}$ with $\mathbb{C}$, and let $D_{\mathbb{C}}$ be the same linear chain as $D$ on $M_{\mathbb{C}}$. If $M_{\mathbb{C}} \backslash D_{\mathbb{C}}$ is simply connected in addition, then one can require the surface $\mathbb{S}$ in Proposition 6.8 to be algebraically simply connected. 


\section{Proof of Main Theorem}

We shall prove the Main Theorem by giving explicit examples using the method in Section 6. In Examples 7.17 .8 below, the necessary tasks are:

- Giving assumptions on $\operatorname{char}(\mathbb{k})$ and $K^{2}$.

- Defining two cubic homogeneous polynomials $\phi_{0}$ and $\phi_{\infty}$ in $\mathbb{Z}[\mathrm{x}, \mathrm{y}, \mathrm{z}]$ and checking

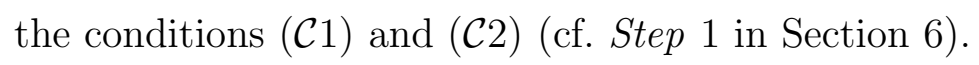

- Defining the divisors $\bar{F}, \bar{S}$, and $\bar{G}$ on $Y$, and checking $(\underline{\mathcal{C} 3})$ and $(\underline{\mathcal{C} 4})$. For the ampleness of $K_{X_{t}}$, we check (A1) or (A2) (cf. Step 2 in Section 6).

- Defining the divisor $B$ on $Z$ after checking $(\underline{\mathcal{C} 5})$ and (다) (cf. Step 3 in Section 6 ).

- Defining the birational morphism $\varphi: M \rightarrow Y$ and the union $D$ of linear chains of

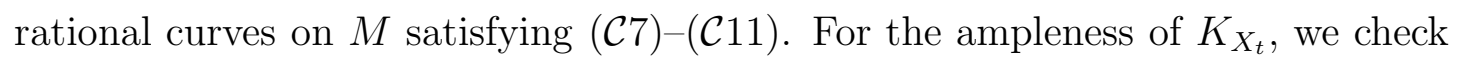
(A3) (cf. Step 4 in Section 6).

- If possible, proving the ampleness of $K_{X}$ (equivalent to the absence of (-2)-curves on $M \backslash D)$ using (A1)-( $(\mathcal{A} 3)$, or another argument (cf. Proposition 6.6. (44)).

- Proving that $M \backslash D$ is simply connected (with respect to the euclidean topology) when $\mathbb{k}=\mathbb{C}$ using Lemma 6.10.1] or referring to papers [33], [42] and [43].

Having done these tasks, we obtain the desired surfaces by Propositions 6.8 and 6.11. The proof of the Main Theorem is written at the end.

Notation. $\quad$ Let $(\mathrm{x}: \mathrm{y}: \mathrm{z})$ be a homogeneous coordinate of $\mathbb{P}^{2}$.

- A $(-k)$-curve means a non-singular rational curve with self-intersection number $-k$.

- The symbol LC $\left(b_{1}, \ldots, b_{l}\right)$ expresses a linear chain $E=\sum_{i=1}^{l} E_{i}$ of smooth rational curves with the dual graph

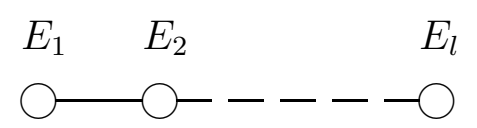

such that $E_{i}^{2}=-b_{i}$ for $1 \leq i \leq l$. Here, $E_{i}$ is called the $i$-th component.

- For a non-zero regular section $\phi$ of an invertible sheaf, $(\phi)_{0}$ denotes the divisor of zeros of $\phi$.

- The configuration type of singular fibers for a minimal elliptic fibration is the list of singular fibers written by Kodaira's symbol (cf. [25, Theorem 6.2]).

Example 7.1. Assume that $\operatorname{char}(\mathbb{k}) \neq 2,3$, and we set $K^{2}=2$. Here, we use essentially the same construction as in [33, Section 3]. We set

$$
\phi_{0}:=\left(3 x y-z^{2}\right) z \text { and } \phi_{\infty}:=(x+y)^{3} .
$$


Then, $\Phi_{0}=\left(\phi_{0}\right)_{0}=Q+L_{1}$ and $\Phi_{\infty}=\left(\phi_{\infty}\right)_{0}=3 L_{2}$ for the conic $Q:=\left(3 \mathrm{xy}-\mathrm{z}^{2}\right)_{0}$ and for the lines $L_{1}:=(\mathrm{z})_{0}$ and $L_{2}:=(\mathrm{x}+\mathrm{y})_{0}$. Thus, (다) holds. Moreover, (드) also holds; In fact, for $c \neq 0$, the divisor $\Phi_{c}=\left(\phi_{0}+c \phi_{\infty}\right)_{0}$ is singular if and only if $c= \pm 1 / 2$, where $\Phi_{1 / 2}$ and $\Phi_{-1 / 2}$ are nodal rational curves with the nodes at $(1:-1: 1)$ and $(1: 1: 1)$, respectively. On the minimal elliptic fibration $\pi: Y \rightarrow \mathbb{P}_{\mathbb{k}}^{1}$ defined by $\Phi$, the configuration type of singular fibers is $\left(\mathrm{IV}^{*}, \mathrm{I}_{2}, \mathrm{I}_{1}, \mathrm{I}_{1}\right)$, and the $(-1)$-curves exceptional for $Y \rightarrow \mathbb{P}_{\mathbb{k}}^{2}$ are mutually disjoint sections $\bar{S}_{1}, \bar{S}_{2}$, and $\bar{S}_{3}$ as in Figure 1 , Let $\bar{F}_{1}$ and $\bar{F}_{2}$ be

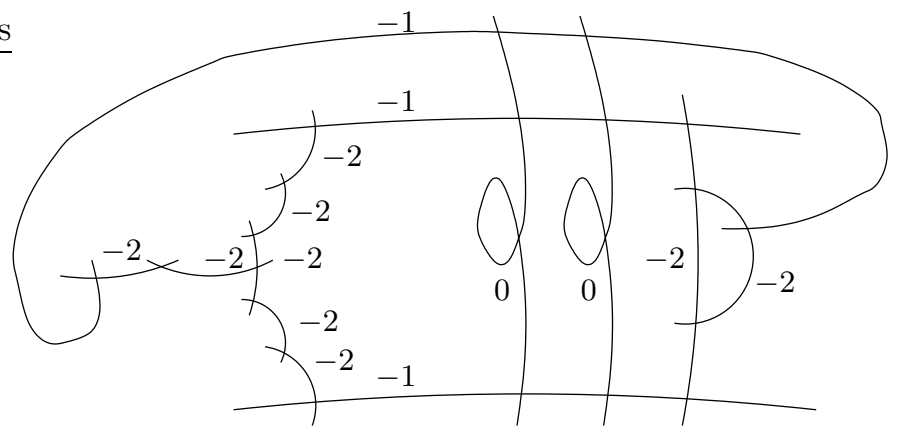

Figure 1. The rational elliptic surface $Y$ in Example 7.1

the proper transforms of $\Phi_{1 / 2}$ and $\Phi_{-1 / 2}$ in $Y$, respectively. These are the singular fibers of type $\mathrm{I}_{1}$, and we define $\bar{F}:=\bar{F}_{1}+\bar{F}_{2}$. The singular fiber of type $\mathrm{I}_{2}$ is the union of the proper transforms $Q^{\sim}$ and $L_{1}^{\sim}$ of $Q$ and $L_{1}$ in $Y$, respectively. The central irreducible component of the singular fiber of type $\mathrm{IV}^{*}$, which meets three other components, is just the proper transform $L_{2}^{\sim}$ of $L_{2}$ in $Y$. We may assume that $\bar{S}_{1}, \bar{S}_{2}$, and $\bar{S}_{3}$ are contracted to the points $(1:-1: \sqrt{-3}),(1:-1:-\sqrt{-3})$, and $(1:-1: 0)$, respectively, where $Q \cap L_{2}=\{(1:-1: \sqrt{-3}),(1:-1:-\sqrt{-3})\}$, and $L_{1} \cap L_{2}=(1:-1: 0)$. Hence, $\bar{S}_{1}$ and $\bar{S}_{2}$ intersect $Q^{\sim}$, and $\bar{S}_{3}$ intersects $L_{1}^{\sim}$. We define $\bar{S}$ to be $\bar{S}_{1}+\bar{S}_{2}+\bar{S}_{3}$. The union $\bar{G}^{+}$of all the $(-2)$-curves on $Y$ is just the union of the singular fibers of type $\mathrm{IV}^{*}$ and $\mathrm{I}_{2}$. Hence, $\bar{B}^{+}=\bar{F}+\bar{G}^{+}+\bar{S}$ satisfies the condition (드). We define $\bar{G}$ to be the union of the $(-2)$-curves except for two $(-2)$-curves: One is $L_{1}^{\sim}$ and the other is the irreducible component of the singular fiber of type $\mathrm{IV}^{*}$ next to the end component meeting $\bar{S}_{1}$. Then,

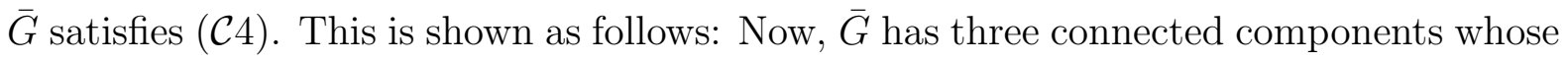
dual graphs are of type $A_{5}, A_{1}$, and $A_{1}$. Hence, $\operatorname{det}\left(\bar{G}_{i} \bar{G}_{j}\right)=-24 \not \equiv 0 \bmod \operatorname{char}(\mathbb{k})$ by $\operatorname{det} \mathrm{A}_{n}=(-1)^{n}(n+1)$. Thus, we have (C4) by Lemma 6.3 .

We shall prove $(\underline{A \mathcal{A} 1})$. Let $V \rightarrow \mathbb{P}_{\mathbb{k}}^{2}$ be the blowing up at the point $(1:-1: \sqrt{-3}) \in$ $Q \cap L_{2}$. Then, $V$ is the Hirzebruch surface of degree one and the proper transform $Q^{\prime}$ of $Q$ in $V$ is ample. For the total transform $L_{1}^{\prime}$ of $L_{1}$ in $V$, we have an isomorphism from $Y \backslash(\bar{S} \cup \bar{G})$ to the affine open subset $V \backslash\left(Q^{\prime} \cup L_{1}^{\prime}\right)$. Thus, ( 
By Figure 1, we see that $(\underline{\mathcal{C} 5})$ is true with the exceptional divisors $J_{1}$ and $J_{2}$ over the nodes, and that $(\underline{\mathcal{C} 6})$ is also true. We define $B:=S+F+G$, i.e., $B$ does not contain $J_{1}$ and $J_{2}$.

We take a birational morphism $\varphi: M \rightarrow Z$ so that the total transform $B_{M}^{+}$of $\bar{B}^{+}$in $M$ as in Figure 2, Note that $\varphi$ is determined uniquely by this figure. A detailed construction

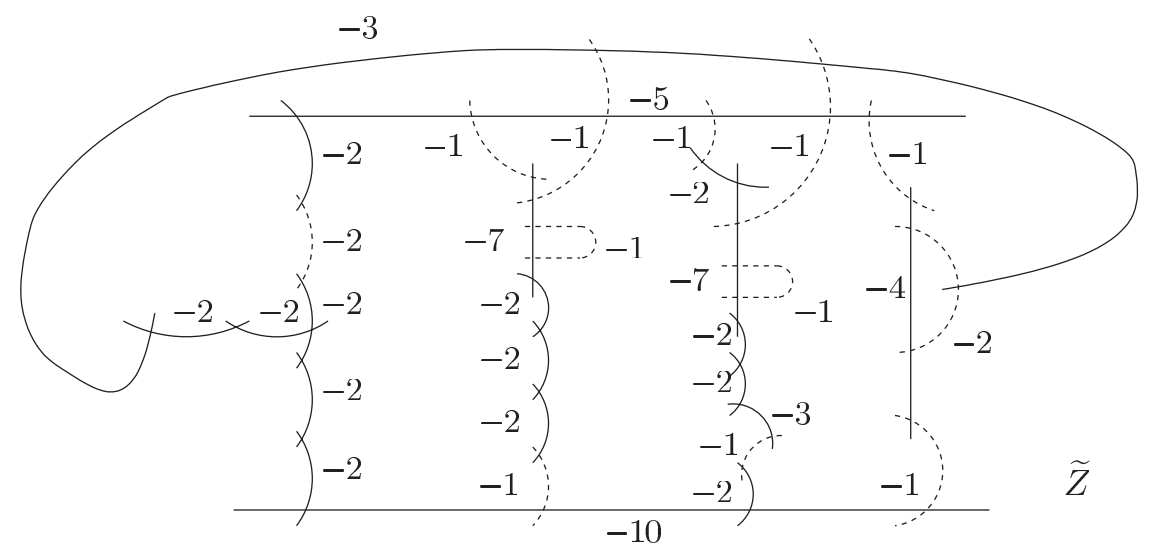

Figure 2. The rational surface $M$ in Example 7.1

of $\varphi$ is written in [33, Section 3]. In particular, $\rho(M)=\rho(Z)+16=28$ and $K_{M}^{2}=-18$. Here, we can find a disjoint union $D$ of the following five linear chains of smooth rational curves in $\varphi^{-1}(B)$ :

$$
\begin{gathered}
D_{1}=\mathrm{LC}(2,10,2,2,2,2,2,3), \quad D_{2}=\mathrm{LC}(2,7,2,2,3), \\
D_{3}=\mathrm{LC}(7,2,2,2), \quad D_{4}=\mathrm{LC}(5,2), \quad D_{5}=\mathrm{LC}(4)
\end{gathered}
$$

Then, (다) is checked by comparing Figures 1 and 2. Moreover, (다) is also true: In fact, $D_{1}, D_{2}, \ldots, D_{5}$ define toric singularities of types $T(1,15,8), T(1,9,5), T(1,5,1)$, $T(1,3,1), T(1,2,1)$, respectively (cf. Table 1). Moreover, using Table 1, we can check (C9) by:

$$
\begin{aligned}
K_{X}^{2} & =K_{M}^{2}+\delta(1,15,8)+\delta(1,9,5)+\delta(1,5,1)+\delta(1,3,1)+\delta(1,2,1) \\
& =-18+8+5+4+2+1=2
\end{aligned}
$$

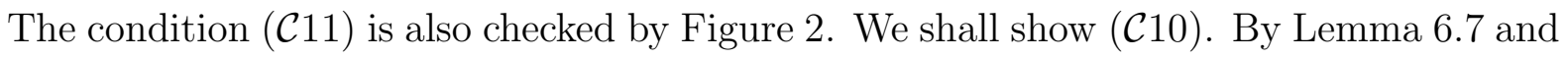
by Figure 2, it suffices to check $\Delta E_{i}>1$ for the (-1)-curves $E_{1}, E_{2}$, and $E_{3}$ characterized by:

- $E_{1}$ joins the (-10)-curve of $D_{1}$ and the end (-2)-component of $D_{3}$.

- $E_{2}$ joins the end (-2)-component of $D_{2}$ and $(-5)$-curve of $D_{4}$.

- $E_{3}$ joins the end (-2)-component of $D_{1}$ and the $(-3)$-curve of $D_{2}$. 
We can calculate $\Delta E_{i}$ for $i=1,2,3$ using Table 1, where the multiplicity $c_{i}$ of the $i$-th irreducible component of the linear chain of type $T(d, n, a)$ equals $1-r_{i} / n$. Thus,

$$
\begin{gathered}
\Delta E_{1}=(1-1 / 15)+(1-4 / 5)>1, \quad \Delta E_{2}=(1-5 / 9)+(1-1 / 3)>1, \text { and } \\
\Delta E_{3}=(1-8 / 15)+(1-4 / 9)>1 .
\end{gathered}
$$

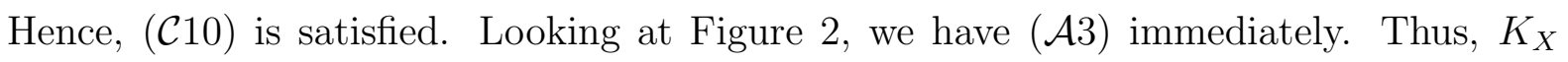

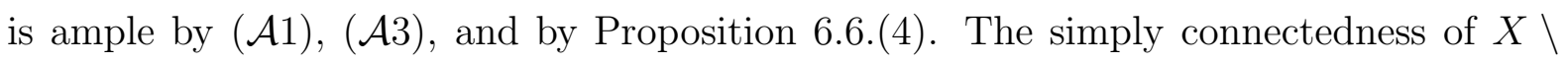
Sing $X \simeq M \backslash D$ has been shown in the proof of [33, Theorem 3.1]. Thus, we have done all the tasks.

Example 7.2. Assume that $\operatorname{char}(\mathbb{k}) \neq 2$ and we set $K^{2}=2$. We follow the construction in [33, Section 6, Construction]. We set

$$
\phi_{0}:=\mathrm{y}^{2} \mathrm{z}-\mathrm{x}^{2}(\mathrm{x}-\mathrm{z}) \quad \text { and } \quad \phi_{\infty}:= \begin{cases}(\mathrm{x}+\mathrm{z}) \mathrm{z}^{2}, & \text { if } \operatorname{char}(\mathbb{k}) \neq 5 \\ (\mathrm{x}+2 \mathrm{z}) \mathrm{z}^{2}, & \text { if } \operatorname{char}(\mathbb{k})=5\end{cases}
$$

Then, $\Phi_{\infty}=\left(\phi_{\infty}\right)_{0}=L_{1}+2 L_{2}$ for the lines $L_{1}=(\mathrm{x}+\mathbf{z})_{0}$ and $L_{2}=(\mathbf{z})_{0}$, and $\Phi_{0}=\left(\phi_{0}\right)_{0}$ is a nodal rational cubic curve with node at $(0: 0: 1)$. Furthermore, $L_{2}$ is the tangent line of $\Phi_{0}$ at an inflection point $(0: 1: 0)=L_{1} \cap L_{2}$. In particular, (드) holds. Moreover, $(\mathcal{C} 2)$ also holds: In fact, we can check the following:

- For $c \neq 0$, the divisor $\Phi_{c}=\left(\phi_{0}+c \phi_{\infty}\right)_{0}$ is singular if and only if $c=(11 \pm 5 \sqrt{5}) / 2$ when $\operatorname{char}(\mathbb{k}) \neq 5$ and $c=1 \pm \sqrt{3}$ when $\operatorname{char}(\mathbb{k})=5$.

- Let $c_{ \pm}$be the constants $(11 \pm 5 \sqrt{5}) / 2$ when $\operatorname{char}(\mathbb{k}) \neq 5$ and $1 \pm \sqrt{3}$ when $\operatorname{char}(\mathbb{k})=$ 5. Then, the node of $\Phi_{c_{ \pm}}$is $(-(1 \pm \sqrt{5}) / 2: 0: 1)$ when $\operatorname{char}(\mathbb{k}) \neq 5$ and $( \pm 2 \sqrt{3}: 0: 1)$ when $\operatorname{char}(\mathbb{k})=5$.

On the minimal elliptic fibration $\pi: Y \rightarrow \mathbb{P}_{\mathbb{k}}^{1}$ defined by $\Phi$, the configuration type of singular fibers is (III* $\left.\mathrm{I}_{1}, \mathrm{I}_{1}, \mathrm{I}_{1}\right)$, and the (-1)-curves exceptional for $Y \rightarrow \mathbb{P}_{\mathbb{k}}^{2}$ are mutually disjoint sections $\bar{S}_{1}, \bar{S}_{2}$, and $\bar{S}_{3}$ as in Figure 3. Let $\bar{F}_{1}$ and $\bar{F}_{2}$ be the proper transforms of $\Phi_{c_{+}}$and $\Phi_{c_{-}}$in $Y$, respectively. These are singular fibers of type $\mathrm{I}_{1}$. We define $\bar{F}:=\bar{F}_{1}+\bar{F}_{2}$. We may assume that $\bar{S}_{1}, \bar{S}_{2}$, and $\bar{S}_{3}$ are contracted to the points $(0: 1: 0)$, $(1: \sqrt{-2}:-1)$, and $(1:-\sqrt{-2}:-1)$, respectively, where $\Phi_{0} \cap L_{2}=L_{1} \cap L_{2}=\{(0: 1: 0)\}$ and $\Phi_{0} \cap L_{1}=\{(0: 1: 0),(1: \pm \sqrt{-2}:-1)\}$. An end component of the singular fiber of type III* is contracted to $(0: 1: 0)$ and meets $\bar{S}_{1}$. Another end component is the proper transform $L_{1}^{\sim}$ of $L_{1}$ in $Y$ and meets $\bar{S}_{2}$ and $\bar{S}_{3}$. The other end component is the proper transform $L_{2}^{\sim}$ of $L_{2}$ in $Y$. We define $\bar{S}:=\bar{S}_{1}+\bar{S}_{2}+\bar{S}_{3}$. The union $\bar{G}^{+}$of $(-2)-$ curves on $Y$ is just the support of the singular fiber of type III*. Thus, (단 holds for $\bar{B}^{+}=\bar{F}+\bar{G}^{+}+\bar{S}$. We define $\bar{G}:=\bar{G}^{+}-L_{2}^{\sim}$. Then, $\bar{G}$ is connected with the dual graph 


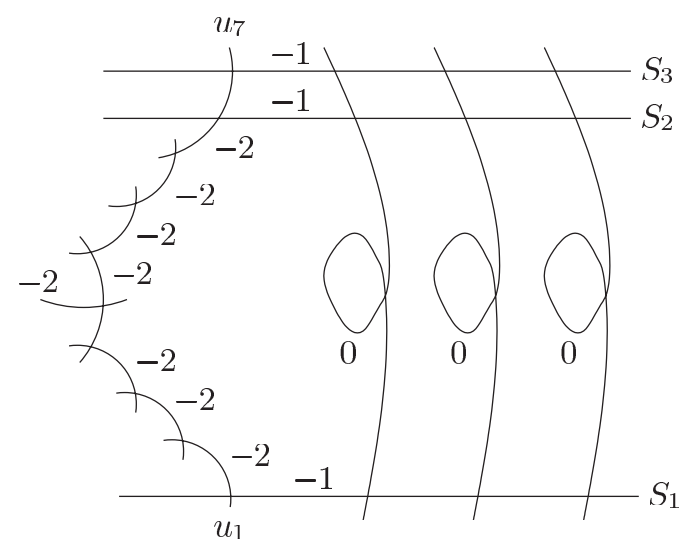

Figure 3. The rational elliptic surface $Y$ in Examples 7.2

A $_{7}$. Thus, $\operatorname{det}\left(\bar{G}_{i} \bar{G}_{j}\right)=-8 \not \equiv 0 \bmod \operatorname{char}(\mathbb{k})$, and hence, we have $(\underline{\mathcal{C} 4})$ by Lemma 6.3 . Furthermore, (스) is true, since $Y \backslash(\bar{S} \cup \bar{G})$ is isomorphic to $\mathbb{P}^{2} \backslash L_{1}$.

We have $(\underline{\mathcal{C} 5})$ and $(\underline{\mathcal{C} 6})$ on $Z$ by looking at Figure 3 . We define $B:=S+F+G$, i.e., $J_{1}$ and $J_{2}$ are not contained in $B$.

We take the birational morphism $\varphi: M \rightarrow Z$ so that the total transform $B_{M}^{+}$of $\bar{B}^{+}$in $M$ as in Figure 4 (cf. [33, Section 6, Fig. 4]). In particular, $\rho(M)=\rho(Z)+20=32$ and

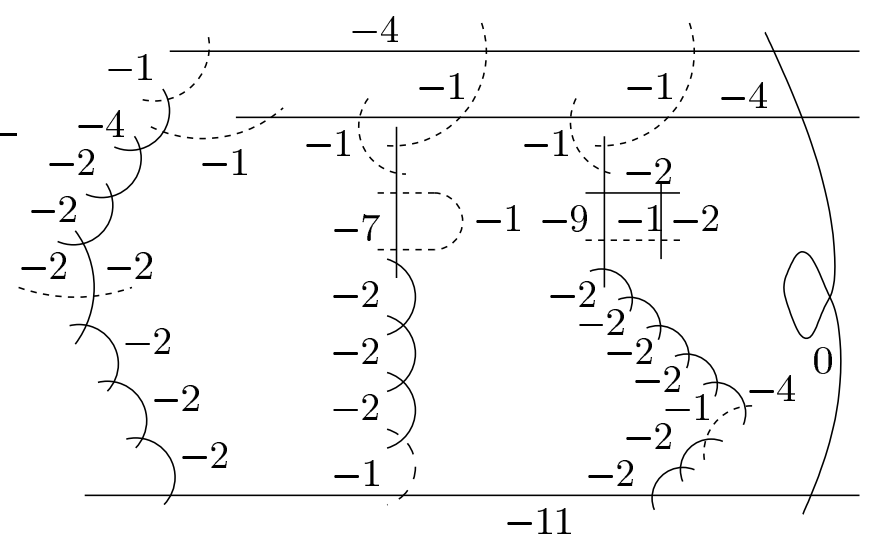

Figure 4. The rational surface $M$ in Example 7.2

$K_{M}^{2}=-22$. Here, we can find a disjoint union $D$ of the following five linear chains of smooth rational curves satisfying (드):

$$
\begin{gathered}
D_{1}=\mathrm{LC}(2,2,11,2,2,2,2,2,2,4), \quad D_{2}=\mathrm{LC}(2,2,9,2,2,2,2,4), \\
D_{3}=\mathrm{LC}(7,2,2,2), \quad D_{4}=\mathrm{LC}(4), \quad D_{5}=\mathrm{LC}(4) .
\end{gathered}
$$

By Tables 1 and 2, we see that the linear chains $D_{1}, \ldots, D_{5}$ define toric singularities of types $T(1,25,17), T(1,19,13), T(1,5,1), T(1,2,1), T(1,2,1)$, respectively. Thus, (C) 
holds. We have (드) from the calculation

$$
\begin{aligned}
K_{X}^{2} & =K_{M}^{2}+\delta(1,25,17)+\delta(1,19,13)+\delta(1,5,1)+2 \delta(1,4,1) \\
& =-22+10+8+4+2=2
\end{aligned}
$$

using Tables 1 and 2, The condition (대) is checked by looking at Figure 4 . We shall

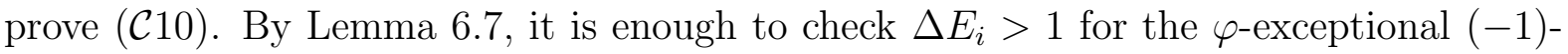
curves $E_{1}, E_{2}, E_{3}$ characterized by:

- $E_{1}$ joins the $(-11)$-curve in $D_{1}$ and the end $(-2)$-curve in $D_{3}$.

- $E_{2}$ joins the (-9)-curve in $D_{2}$ and the end (-2)-curve of $D_{2}$.

- $E_{3}$ joins $(-4)$-curve in $D_{2}$ and the end $(-2)$-curve in $D_{1}$.

Then, by Tables 1 and 2 , we have:

$$
\begin{gathered}
\Delta E_{1}=(1-1 / 25)+(1-4 / 5)>1, \quad \Delta E_{2}=(1-1 / 19)+(1-13 / 19)>1, \\
\Delta E_{3}=(1-6 / 19)+(1-17 / 25)>1 .
\end{gathered}
$$

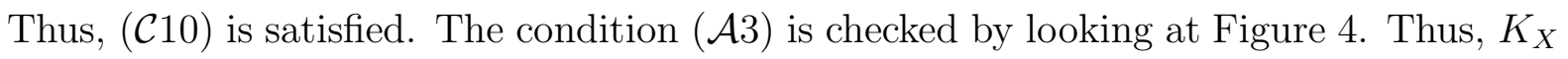

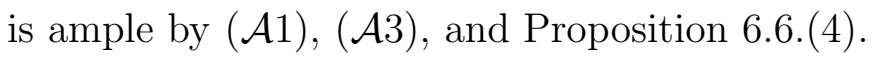

We shall prove that $M \backslash D$ is simply connected when $\mathbb{k}=\mathbb{C}$ (the proof is omitted in [33, Section 6]). We apply Lemma 6.10.1. Let $T\left(d_{i}, n_{i}, a_{i}\right)$ be the type of the singular point defined by $D_{i}$. Then, $d_{1}=\cdots=d_{5}=1$ and $\left(n_{1}, \ldots, n_{5}\right)=(25,19,5,2,2)$. Looking at Figure 4, we have:

- the (-1)-curve $E_{3}$ which meets end components of $D_{1}$ and $D_{2}$.

- a $(-1)$-curve meeting the end $(-4)$-component of $D_{1}$ and $D_{4}\left(\operatorname{resp} . D_{5}\right)$.

- a $(-1)$-curve meeting the end $(-7)$-component of $D_{3}$ and $D_{4}$.

Since $\operatorname{gcd}\left(n_{1}, n_{2}\right)=\operatorname{gcd}(25,19)=1, \operatorname{gcd}\left(n_{1}, n_{4}\right)=\operatorname{gcd}\left(n_{1}, n_{5}\right)=\operatorname{gcd}(25,2)=1$, and $\operatorname{gcd}\left(n_{3}, n_{4}\right)=\operatorname{gcd}(5,2)=1$, the conditions of Lemma 6.10.1 are all satisfied, and hence $M \backslash D$ is simply connected. Therefore, we have done all the tasks.

Remark 7.2.1. It is known by [22] that there exist rational elliptic surfaces whose singular fibers are of configuration type (III*, $\left.\mathrm{I}_{1}, \mathrm{I}_{1}, \mathrm{I}_{1}\right)$ in characteristic 3 .

Example 7.3. Assume that $\operatorname{char}(\mathbb{k}) \neq 2$ and set $K^{2}=1$. We consider the same cubic

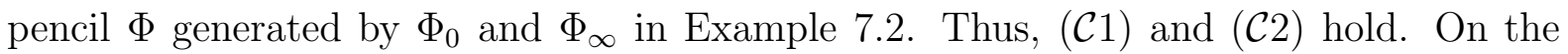
elliptic fibration $Y \rightarrow \mathbb{P}_{\mathbb{k}}^{1}$, let $\bar{F}=\bar{F}_{1}+\bar{F}_{2}$ and $\bar{S}=\bar{S}_{1}+\bar{S}_{2}+\bar{S}_{3}$ be the same as in Example 17.2, but here we define $\bar{G}$ to be $\bar{G}^{+}$minus two irreducible components of the singular fiber of type III*; one is $L_{2}^{\sim}$ and the other is the component next to $L_{1}^{\sim}$. Then,

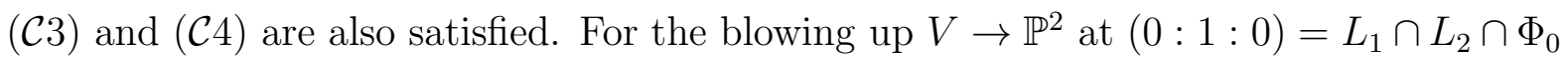
and for the proper transform $L_{1}^{\prime}$ of $L_{1}$ in $V$, we see that $Y \backslash(\bar{S} \cup \bar{G})$ is isomorphic to $V \backslash L_{1}^{\prime}$ 
minus a point, which is the image of $\bar{S}_{1}$. In particular, the condition (슬 is not satisfied. However, any projective curve contained in $Y \backslash(\bar{S} \cup \bar{G})$ is a non-singular rational curve having the self-intersection number zero and is mapped to a line of $\mathbb{P}_{\mathbb{k}}^{2}$ passing through $(0: 1: 0)$.

We have $(\underline{\mathcal{C} 5})$ and $(\underline{\mathcal{C} 6})$ by Figure 3 , We define $B:=S+G+F$.

We take the birational morphism $\varphi: M \rightarrow Z$ by the following steps: (ii)-(iii):

(i) First, we blow up at the two intersection points $F_{1} \cap S_{2}$ and $F_{1} \cap S_{3}$.

(ii) We blow up 4 times successively at the intersection point of $F_{1}$ and the proper transform of $S_{1}$. Then, we have a linear chain $\operatorname{LC}(7,2,2,2)$ of rational curves.

(iii) Applying the same process in (ii) and (iii) to $F_{2}$, we obtain a rational surface $M$ with $\rho(M)=\rho(Z)+12=24$ and $K_{M}^{2}=-14$.

Then, the total transform $B_{M}^{+}$of $B^{+}$is as in Figure 5 ,

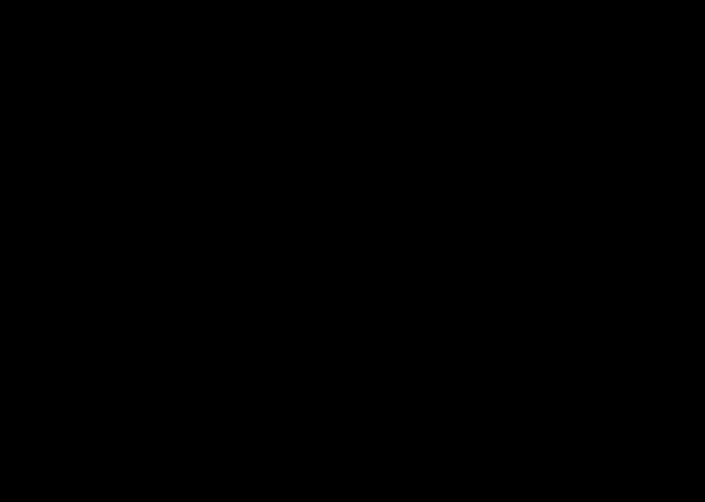

FiguRE 5. The rational surface $M$ in Example 7.3

Here, we see that $M$ has a disjoint union $D$ of the following four linear chains of smooth rational curves:

$$
\begin{gathered}
D_{1}=\mathrm{LC}(7,2,2,2) \text { over } F_{1}, \quad D_{2}=\mathrm{LC}(7,2,2,2) \text { over } F_{2}, \\
D_{3}=\mathrm{LC}(3,2,3), \quad D_{4}=\mathrm{LC}(9,2,2,2,2,2) .
\end{gathered}
$$

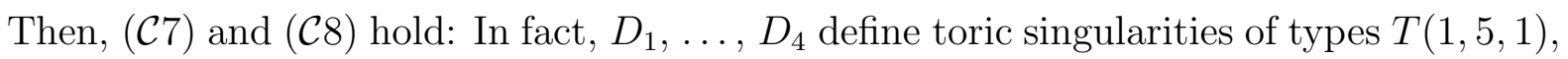
$T(1,5,1), T(3,2,1), T(1,7,1)$, respectively (cf. Table 1). We have (드) by the calculation

$$
K_{X}^{2}=K_{M}^{2}+2 \delta(1,5,1)+\delta(3,2,1)+\delta(1,7,1)=-14+8+1+6=1
$$

using Table 1. We can check ( $(\underline{\mathcal{C} 11})$ and $(\underline{\mathcal{A} 3})$ by Figure 5 . We shall prove $(\underline{\mathbb{C} 10})$. By Lemma 6.7, it is enough to show $\Delta E_{i}>1$ for $i=1,2$ for the (-1)-curves $E_{1}, E_{2}$ such that $E_{i}$ joins the (-9)-curve of $D_{4}$ and the end (-2)-curve of $D_{i}$ for $i=1,2$. By using Table 1, we have

$$
\Delta E_{1}=\Delta E_{2}=(1-1 / 7)+(1-4 / 5)>1 .
$$


Thus, (10) holds. The ampleness of $K_{X}$ is proved as follows. Assuming the contrary, we have a (-2)-curve $\Gamma$ contained in $M \backslash D$ by Proposition 6.6. (44). Then, $\Gamma$ is not $\varphi$ exceptional by ( $(\underline{\mathcal{A} 3})$, and the projective curve $\bar{\Gamma}:=\tau(\varphi(\Gamma))$ is contained in $Y \backslash(\bar{G} \cup \bar{S})$. By the property of this open subset of $Y$ discussed above, $\bar{\Gamma}$ is a non-singular rational curve with self-intersection number zero and the image of $\bar{\Gamma}$ in $\mathbb{P}_{\mathbb{k}}^{2}$ is a line $L$ passing through $(0: 1: 0)$. Since $\varphi(\Gamma)$ is a $(-2)$-curve, $\bar{\Gamma}$ is a bisection of $Y \rightarrow \mathbb{P}^{1}$ passing through the nodes of $\bar{F}_{1}$ and $\bar{F}_{2}$. Hence, $L$ is a line passing through the nodes of $\Phi_{c_{ \pm}}$; thus, $L=(\mathrm{y})_{0}$. This contradicts $L \ni(0: 1: 0)$. Therefore, $K_{X}$ is ample.

We shall show that $M \backslash D$ is simply connected when $\mathbb{k}=\mathbb{C}$. We apply Lemma 6.10.1. Let $T\left(d_{i}, n_{i}, a_{i}\right)$ be the type of $D_{i}$ for $1 \leq i \leq 4$. Then, $\left(d_{1}, \ldots, d_{4}\right)=(1,1,3,1)$ and $\left(n_{1}, \ldots, n_{4}\right)=(5,5,2,7)$. Looking at Figure 5, we have:

- the (-1)-curve $E_{4}$ which meets end components of $D_{1}$ and $D_{4}$.

- the (-1)-curve $E_{2}$ which meets end components of $D_{2}$ and $D_{4}$.

- a $(-1)$-curve meeting the end (-3)-component of $D_{3}$ and the end $(-7)$-component of $D_{1}$.

Since $\operatorname{gcd}\left(d_{1} n_{1}, d_{4} n_{4}\right)=\operatorname{gcd}\left(d_{2} n_{2}, d_{4} n_{4}\right)=\operatorname{gcd}(5,7)=1$ and $\operatorname{gcd}\left(d_{1} n_{1}, d_{3} n_{3}\right)=\operatorname{gcd}(5,6)$ $=1$, the conditions of Lemma 6.10.1 are all satisfied, and hence $M \backslash D$ is simply connected. Therefore, we have done all the tasks.

Example 7.4. Assume that $\operatorname{char}(\mathbb{k}) \neq 2$, and we set $K^{2}=3$. We follow the construction in [42, Section 3]. We set

$$
\phi_{0}:=\left(\mathrm{xy}+\mathrm{z}^{2}\right)(\mathrm{x}+\mathrm{y}) \text { and } \phi_{\infty}:=\mathrm{xyz} .
$$

Then, $\Phi_{0}=Q+L_{4}$ and $\Phi_{\infty}=L_{1}+L_{2}+L_{3}$ for the conic $Q=\left(\mathrm{xy}+\mathrm{z}^{2}\right)_{0}$, and the lines $L_{1}=(\mathrm{x})_{0}, L_{2}=(\mathrm{y})_{0}, L_{3}=(\mathrm{z})_{0}$, and $L_{4}=(\mathrm{x}+\mathrm{y})_{0}$. In particular, (투) holds, and

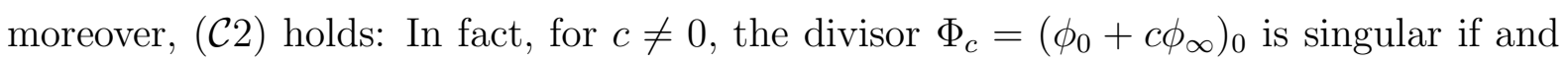
only if $c= \pm 4$, where $\Phi_{4}$ and $\Phi_{-4}$ are nodal rational curves with the nodes at $(1: 1:-1)$ and $(1: 1: 1)$, respectively. On the minimal elliptic fibration $\pi: Y \rightarrow \mathbb{P}_{\mathbb{k}}^{1}$ defined by $\Phi$, the configuration type of singular fibers is $\left(\mathrm{I}_{8}, \mathrm{I}_{2}, \mathrm{I}_{1}, \mathrm{I}_{1}\right)$, and the $(-1)$-curves exceptional for $Y \rightarrow \mathbb{P}_{\mathbb{k}}^{2}$ are mutually disjoint four sections $\bar{S}_{1}, \ldots, \bar{S}_{4}$ as in Figure 6 (cf. [42, Section 3, Figure 2] for $E(1)$ (where the symbols are different from ours)). Let $\bar{F}_{1}$ and $\bar{F}_{2}$ be the proper transforms of $\Phi_{4}$ and $\Phi_{-4}$, respectively. These are the two singular fibers of type $\mathrm{I}_{1}$. We define $\bar{F}:=\bar{F}_{1}+\bar{F}_{2}$. The singular fiber of type $\mathrm{I}_{2}$ is the union of the proper transforms $Q^{\sim}$ and $L_{4}^{\sim}$ of $Q$ and $L_{4}$ in $Y$. The singular fiber of type $\mathrm{I}_{8}$ contains the proper transforms $L_{1}^{\sim}, L_{2}^{\sim}$, and $L_{3}^{\sim}$ of the lines $L_{1}, L_{2}$, and $L_{3}$ in $Y$. The irreducible components of the singular fiber of type $I_{8}$ is labelled as $\Gamma_{0}+\Gamma_{1}+\cdots+\Gamma_{7}$ as a cyclic chain, where we set $L_{1}^{\sim}=\Gamma_{1}, L_{2}^{\sim}=\Gamma_{7}$, and $L_{3}^{\sim}=\Gamma_{4}$. We may assume that $\bar{S}_{1}, \bar{S}_{2}, \bar{S}_{3}$, 


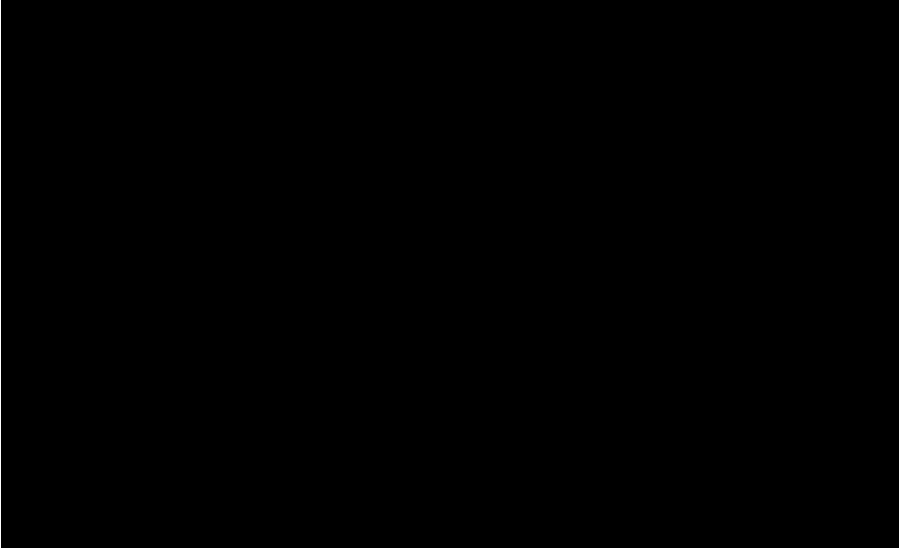

Figure 6. The rational elliptic surface $Y$ in Example 7.4

and $\bar{S}_{4}$ are contracted to the points $(0: 0: 1),(0: 1: 0),(1: 0: 0)$, and $(1:-1: 0)$, respectively, where $L_{1} \cap L_{2} \cap L_{4}=\{(0: 0: 1)\}, L_{1} \cap Q=L_{1} \cap L_{3}=\{(0: 1: 0)\}$, $L_{2} \cap Q=L_{2} \cap L_{3}=\{(1: 0: 0)\}$, and $L_{3} \cap L_{4}=\{(1:-1: 0)\}$. Hence, $\bar{S}_{1}$ intersects $L_{4}^{\sim}$ and $\Gamma_{0} ; \bar{S}_{2}$ intersects $Q^{\sim}$ and $\Gamma_{2} ; \bar{S}_{3}$ intersects $Q^{\sim}$ and $\Gamma_{6} ; \bar{S}_{4}$ intersects $L_{4}^{\sim}$ and $L_{3}^{\sim}=\Gamma_{4}$.

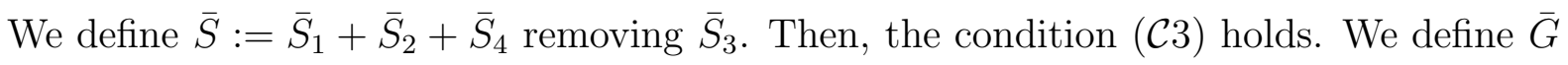
to be

$$
\bar{G}^{+}-\Gamma_{3}-Q^{\sim}=\sum_{0 \leq i \leq 7, i \neq 3} \Gamma_{i}+L_{4}^{\sim}
$$

Then, $\bar{G}$ has two connected components whose dual graphs are $\mathrm{A}_{7}$ and $\mathrm{A}_{1}$. Thus, $\operatorname{det}\left(\bar{G}_{i} \bar{G}_{i}\right)=16 \not \equiv 0 \bmod \operatorname{char}(\mathbb{k})$, and hence $\left(\mathcal{C}_{4}\right)$ is satisfied by Lemma 6.3. For $(\underline{A 1})$, since $\rho(Y)=10$, it is enough to prove that $\bar{S}_{1}, \bar{S}_{2}$, and the irreducible components of $\bar{G}$ are linearly independent in $\operatorname{Pic}(Y)$. Assume that

$$
a_{1} \bar{S}_{1}+a_{2} \bar{S}_{2}+a_{3} L_{4}^{\sim}+\sum_{0 \leq i \leq 7, i \neq 3} m_{i} \Gamma_{i} \sim 0
$$

for integers $a_{j}$ and $m_{i}$. Then, calculating the intersection numbers with $Q^{\sim}, L_{4}^{\sim}, \bar{S}_{2}, \bar{S}_{4}$, and $\Gamma_{3}$, we have:

$$
a_{2}+2 a_{3}=a_{1}-2 a_{3}=-a_{2}+m_{2}=a_{3}+m_{4}=m_{2}+m_{4}=0 .
$$

In particular, $a_{1}=a_{2}=a_{3}=m_{2}=m_{4}=0$. The other $m_{j}$ are all zero, since $\operatorname{det}\left(\bar{G}_{i} \bar{G}_{j}\right) \neq$ 0 . Thus, we have the linear independence, and hence (A1).

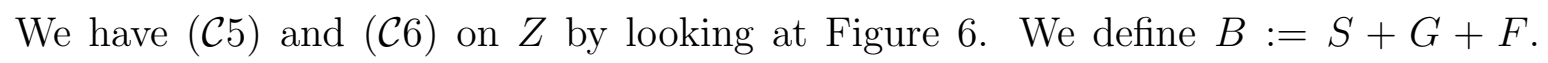
We take the birational morphism $\varphi: M \rightarrow Y$ so that the total transform $B_{M}^{+}$of $\bar{B}^{+}$in $M$ as in Figure 7 (cf. [42, Section 3, Figure 5]). In particular, $\rho(M)=\rho(Z)+19$ and $K_{M}^{2}=-21$. We have a disjoint union $D$ of the following linear chains of smooth rational 


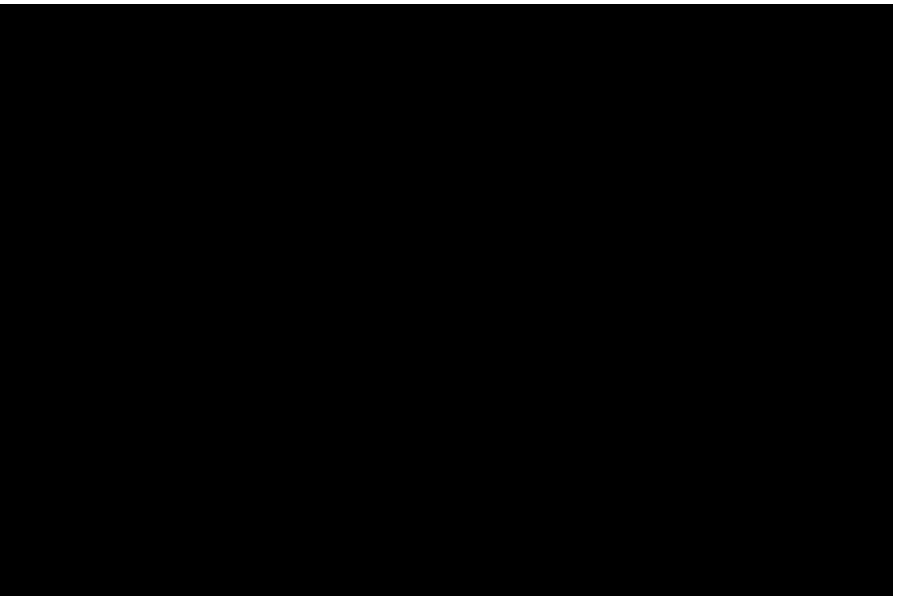

FiguRE 7. The rational surface $M$ in Example 7.4

curves in $\varphi^{-1}(B)$ :

$$
\begin{gathered}
D_{1}=\mathrm{LC}(6,8,2,2,2,3,2,2,2,2), \quad D_{2}=\mathrm{LC}(4), \\
D_{3}=\mathrm{LC}(4,7,2,2,3,2,2), \quad D_{4}=\mathrm{LC}(9,2,2,2,2,2) .
\end{gathered}
$$

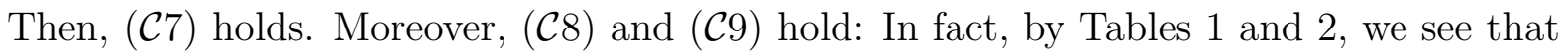
$D_{1}, \ldots D_{4}$ define toric singularities of type $T(1,35,6), T(1,2,1), T(1,15,9), T(1,7,1)$, respectively, and we calculate

$$
K_{X}^{2}=K_{M}^{2}+\delta(1,35,6)+\delta(1,2,1)+\delta(1,15,9)+\delta(1,7,1)=-21+10+1+7+6=3
$$

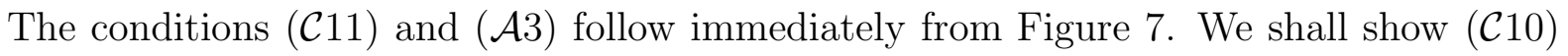
using Lemma 6.7. Then, it suffices to prove $\Delta E_{i}>1$ for the (-1)-curves $E_{1}, E_{2}$, and $E_{3}$ determined by:

- $E_{1}$ joins the $(-7)$-curve in $D_{3}$ and the end $(-2)$-component of $D_{1}$.

- $E_{2}$ joins the $(-9)$-curve in $D_{4}$ and the end $(-2)$-component of $D_{3}$.

- $E_{3}$ joins the (-8)-curve in $D_{1}$ and the end (-2)-component of $D_{4}$.

Then, by Tables 1 and 2, we have:

$$
\begin{gathered}
\Delta E_{1}=(1-1 / 19)+(1-29 / 35)>1, \quad \Delta E_{2}=(1-1 / 7)+(1-14 / 19)>1, \\
\Delta E_{3}=(1-1 / 35)+(1-6 / 7)>1 .
\end{gathered}
$$

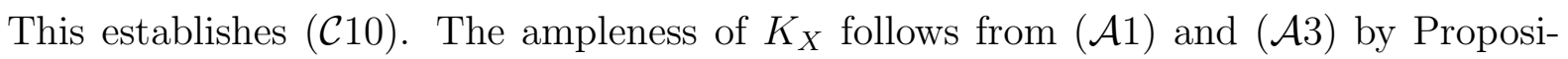
tion 6.6. (44). The simply connectedness of $M \backslash D$ has been shown in the proof of [42, Theorem 3.1]. Thus, we have done all the tasks. 
Remark 7.4.1. It is known by [22] that there exist rational elliptic surfaces whose singular fibers are of configuration type $\left(\mathrm{I}_{8}, \mathrm{I}_{1}, \mathrm{I}_{1}, \mathrm{I}_{2}\right)$ in characteristic 3 . In characteristic 2 , this configuration does not exist by [29].

Example 7.5. Assume that $\operatorname{char}(\mathbb{k}) \neq 2$, and we set $K^{2}=4$. We follow the construction in [43, Section 2]. Here, we define $\phi_{0}$ and $\phi_{\infty}$ to be the same as in Example 17.4. Thus,

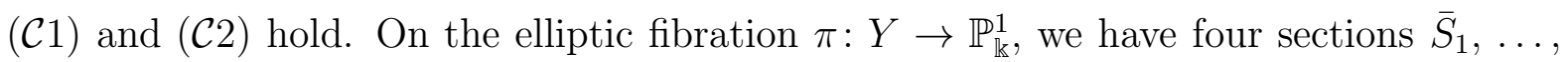
$\bar{S}_{4}$ in Example 17.4, but here, consider another horizontal curve $N^{\sim}$ which is the proper transform of the line $N:=(\mathrm{x}+\mathrm{y}+2 \mathrm{z})_{0}$ in $Y$. Note that $N$ contains $(1:-1: 0)=L_{3} \cap L_{4}$ and the node $(1: 1:-1)$ of $\Phi_{4}$. We define $\bar{S}$ to be $\bar{S}_{3}+\bar{S}_{4}+N^{\sim}$. We also set $\bar{F}=\bar{F}_{1}+\bar{F}_{2}$ as in Example 7.4. Then, we can check (․․․

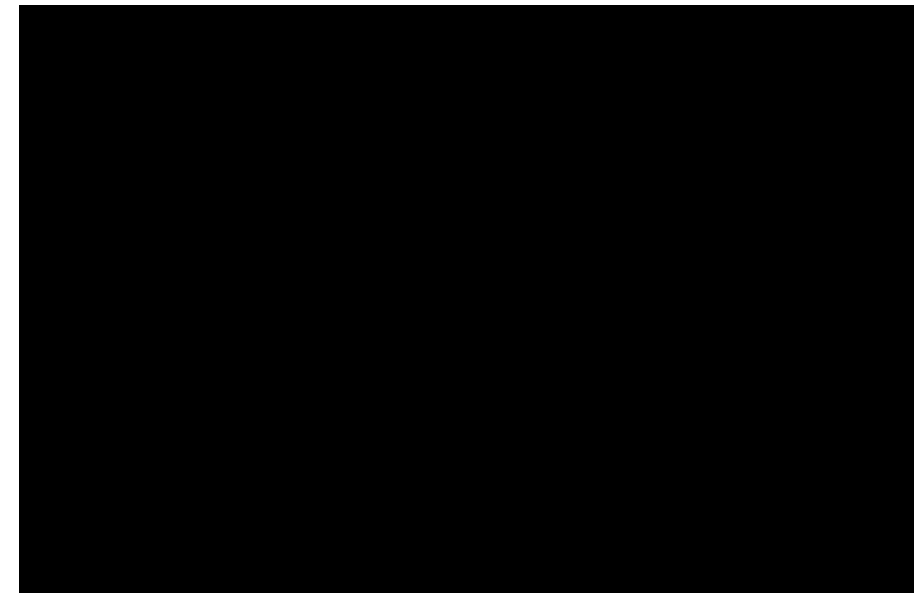

Figure 8. The rational elliptic surface $Y$ in Example 7.5

the divisor $\bar{G}$ to be

$$
\bar{G}^{+}-\Gamma_{0}-L_{2}^{\sim}-Q^{\sim}=\sum_{i=1}^{6} \Gamma_{i}+L_{4}^{\sim}
$$

Note that $\Gamma_{1}=L_{1}^{\sim}, \Gamma_{4}=L_{3}^{\sim}$, and $\Gamma_{7}=L_{2}^{\sim}$. We shall prove (C4) not using Lemma 6.3. Assume that we have a linear equivalence relation

$$
\sum_{i=1}^{6} m_{i} \bar{G}_{i}+m_{7} L_{4}^{\sim}+m_{8} \bar{F}_{1} \sim p H
$$

for integers $m_{1}, \ldots, m_{8}, p=\operatorname{char}(\mathbb{k})$, and a Cartier divisor $H$ on $Y$. Considering the intersection numbers with $Q^{\sim}$ and $\bar{S}_{j}$, we have

$$
p H Q^{\sim}=m_{7}, \quad p H \bar{S}_{1}=m_{7}+m_{8}, \quad p H \bar{S}_{2}=m_{2}+m_{8}, \quad \text { and } \quad p H \bar{S}_{3}=m_{6}+m_{8},
$$

which imply $m_{2} \equiv m_{6} \equiv m_{7} \equiv m_{8} \equiv 0 \bmod p$. Moreover, we have $m_{i-1}+m_{i+1} \equiv 2 m_{i}$ $\bmod p$ for $2 \leq i \leq 6$ by calculating $p H \Gamma_{i}$. Thus, $m_{i} \equiv 0 \bmod p$ for any $i$, since $p \neq 2$.

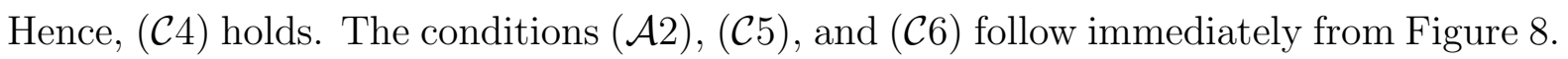


We define $B:=S+G+F+J_{1}$, where we set $\bar{F}_{1}$ to be the proper transform of $\Phi_{4}$ in $Y$. Note that $N^{\sim}$ passes through the node of $\bar{F}_{1}$.

We take the birational morphism $\varphi: M \rightarrow Y$ by the same process as in [43, Section 2] for constructing $Z=Y \sharp 9 \overline{\mathbb{P}}^{2}$ from $Y$, where the symbol $Y \sharp 9 \overline{\mathbb{P}}^{2}$ in 43 . stands for a blown up surface of $Y$ at nine (infinitely near) points. Thus, $\rho(M)=\rho(Z)+7=19, K_{M}^{2}=-9$, and the total transform $B_{M}^{+}$of $\bar{B}^{+}$in $M$ has a configuration in Figure 9 (cf. [43, Figure 3]). Here, we can find a linear chain

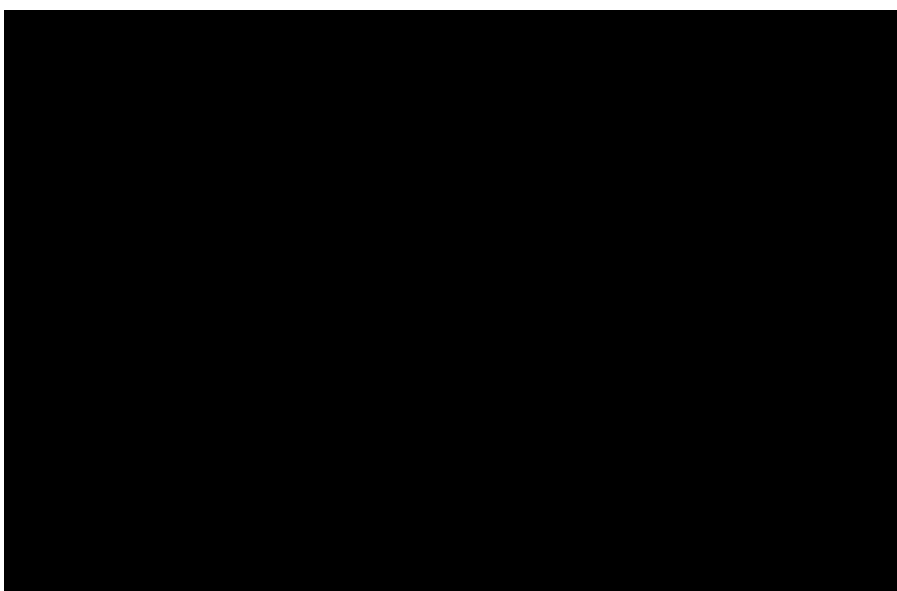

FiguRE 9. The rational surface $M$ in Example 7.5

$$
D=\mathrm{LC}(2,4,6,2,6,2,4,2,2,2,3,2,3)
$$

of smooth rational curves satisfying (들 , which corresponds to the solid lines of Figure 9 , By Table 2, we see that $D$ defines a toric singularity of type $T(1,252,145)$, and

$$
K_{M}^{2}=K_{X}^{2}+\delta(1,252,145)=-9+13=4 .
$$

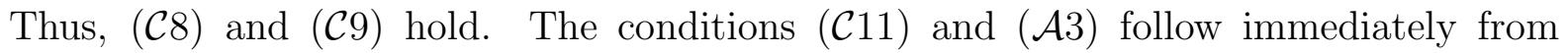
Figure 9, We shall prove ( $(\underline{\mathcal{C} 10})$ by using Lemma 6.7. Then, it suffices to show $\Delta E>1$ for the (-1)-curve $E$ which joins the end (-2)-component and the end (-3)-component of $D$. We have

$$
\Delta E=(1-145 / 252)+(1-107 / 252)>1
$$

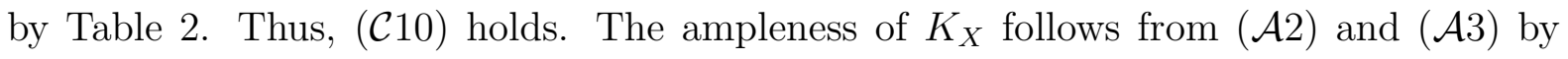
Proposition 6.6. (4), since $B \supset J_{1}$. The simply connectedness of $M \backslash D$ has been shown in the proof of [43, Proposition 2.1]. Thus, we have done all the tasks.

Example 7.6. Assume that $\operatorname{char}(\mathbb{k}) \neq 3$ and we set $K^{2}=1$. We define

$$
\phi_{0}=\mathrm{x}^{2} \mathrm{y}+\mathrm{y}^{2} \mathrm{z}+\mathrm{z}^{2} \mathrm{x} \text { and } \phi_{\infty}=3 \mathrm{xyz}
$$


Then, $\Phi_{\infty}=\left(\phi_{\infty}\right)_{0}=L_{1}+L_{2}+L_{3}$ for the lines $L_{1}=(\mathrm{x})_{0}, L_{2}=(\mathrm{y})_{0}, L_{3}=(\mathrm{z})_{0}$, and $\Phi_{0}=\left(\phi_{0}\right)_{0}$ is a smooth cubic curve such that

$$
\left.\Phi_{0}\right|_{L_{1}}=P_{2}+2 P_{3},\left.\quad \Phi_{0}\right|_{L_{2}}=2 P_{1}+P_{3},\left.\quad \Phi_{0}\right|_{L_{3}}=P_{1}+2 P_{2}
$$

where $P_{1}=(1: 0: 0), P_{2}=(0: 1: 0)$, and $P_{3}=(0: 0: 1)$. In particular, (다) holds, and moreover, $(\underline{\mathcal{C} 2})$ holds: In fact, since $\operatorname{char}(\mathbb{k}) \neq 3$, for $c \neq 0, \Phi_{c}=\left(\phi_{0}+c \phi_{\infty}\right)_{0}$ is singular if and only if $c=-\omega^{i}$ for $i=0,1,2$, where $\omega$ is a primitive cubic root of 1 , and $\Phi_{-\omega^{i}}$ is a nodal rational curve with the node at $\left(1: \omega^{i}: \omega^{-i}\right)$. We also define $N$ to be the line passing through $(0: 1: 0)=L_{1} \cap L_{3}$ and the node $(1: 1: 1)$ of $\Phi_{-1}$. Hence, $N=(\mathbf{x}-\mathbf{z})_{0}$. Thus, we have Figure 10, On the minimal elliptic fibration $\pi: Y \rightarrow \mathbb{P}_{\mathbb{k}}^{1}$ defined by $\Phi$, the

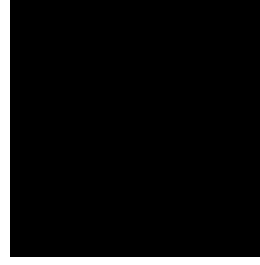

Figure 10. A pencil of cubics in Examples 7.6 and 7.7

configuration type of singular fibers is $\left(\mathrm{I}_{9}, \mathrm{I}_{1}, \mathrm{I}_{1}, \mathrm{I}_{1}\right)$, and the $(-1)$-curves exceptional for $Y \rightarrow \mathbb{P}_{\mathbb{k}}^{2}$ are mutually disjoint three sections $\bar{S}_{1}, \bar{S}_{2}$, and $\bar{S}_{3}$ as in Figure 11. Here, the

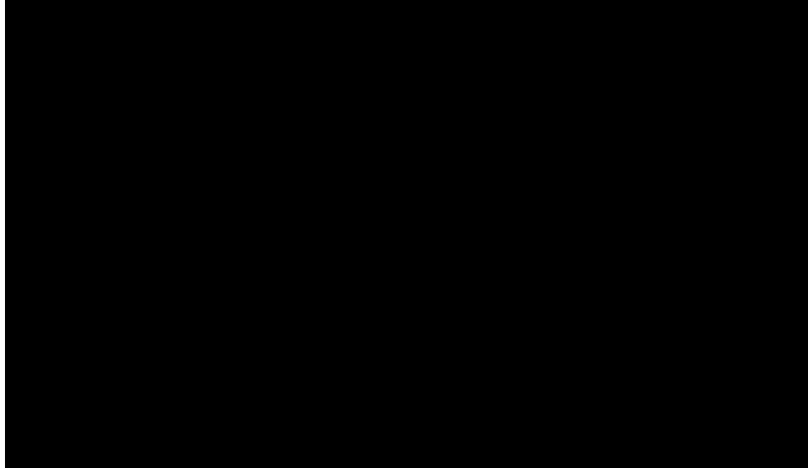

Figure 11. The rational elliptic surface $Y$ in Examples 7.6 and 7.7

singular fibers of type $\mathrm{I}_{1}$ are the proper transforms of $\Phi_{-\omega^{i}}$ for $i=0,1,2$. We set $\bar{F}_{1}$ and $\bar{F}_{2}$ to be the proper transforms of $\Phi_{-1}$ and $\Phi_{-\omega}$, respectively, and define $\bar{F}:=\bar{F}_{1}+\bar{F}_{2}$. The irreducible components of the singular fiber of type $I_{9}$ are labeled as $\Gamma_{1}+\cdots+\Gamma_{9}$ as a cyclic chain of smooth rational curves, where $\Gamma_{3 i}$ is the proper transform of $L_{i}^{\sim}$ for $i=1,2,3$. We may assume that $\bar{S}_{j}$ is contracted to $P_{j}$ by $Y \rightarrow \mathbb{P}_{\mathbb{k}}^{2}$ for $j=1,2,3$. Then,

$$
\bar{S}_{j} \Gamma_{i}= \begin{cases}1, & \text { if } i \equiv 3 j+4 \quad \bmod 9 \\ 0, & \text { otherwise }\end{cases}
$$


The proper transform $N^{\sim}$ of $N$ in $Y$ is a bisection of $\pi: Y \rightarrow \mathbb{P}_{\mathbb{k}}^{1}$ with self-intersection number zero and passing through the node of $\bar{F}_{1}$ but no nodes of other singular fibers of type $\mathrm{I}_{1}$. We have

$$
N^{\sim} \bar{S}_{j}=N^{\sim} \Gamma_{2}-1=N^{\sim} \Gamma_{6}-1=N^{\sim} \Gamma_{i}=0
$$

for any $1 \leq j \leq 3$ and $1 \leq i \neq 2,6 \leq 9$. We define $\bar{S}:=\bar{S}_{2}+N^{\sim}$. Since the union $\bar{G}^{+}$of

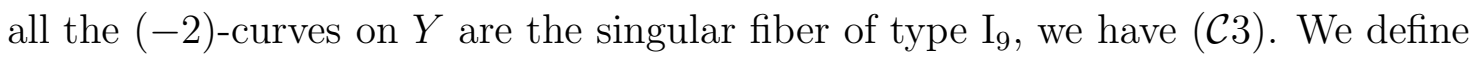

$$
\bar{G}:=\bar{G}^{+}-\Gamma_{3}-\Gamma_{4}-\Gamma_{5}-\Gamma_{8}-\Gamma_{9}=\bar{\Gamma}_{1}+\bar{\Gamma}_{2}+\bar{\Gamma}_{6}+\bar{\Gamma}_{7} .
$$

Then, $\bar{G}$ has two connected components with the dual graph $\mathrm{A}_{2}$. Hence, $\operatorname{det}\left(\bar{G}_{i} \bar{G}_{j}\right) \not \equiv 0$

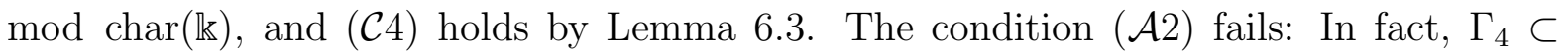
$Y \backslash(\bar{S} \cup \bar{G})$. Thus, it is impossible to require $K_{X}$ to be ample by Proposition 6.6. (4). The conditions $(\underline{\mathcal{C} 5})$ and $(\underline{\mathcal{C} 6})$ on $Z$ follow immediately from Figure 11, We define $B:=$ $S+G+F+J_{1}$. Note that $N^{\sim}$ passes through the node of $\bar{F}_{1}$.

We take the birational morphism $\varphi: M \rightarrow Z$ so that the total transforms of $B+J_{2}$ and $\bar{G}^{+}$in $M$ form a configuration of curves as in Figure 12, Then, $\rho(M)=\rho(Z)+5=17$

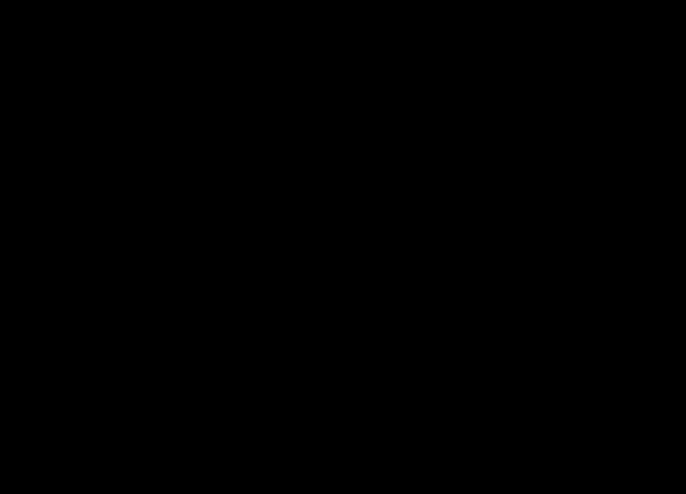

Figure 12. The rational surface $M$ in Example 7.6

and $K_{M}^{2}=-7$. Here, we have a disjoint union $D$ of the following three linear chains of rational curves satisfying (드):

$$
D_{1}=\mathrm{LC}(4,5,3,2,2), \quad D_{2}=\mathrm{LC}(5,2), \quad D_{3}=\mathrm{LC}(3,3) .
$$

By Tables 1 and $2, D_{1}, D_{2}$, and $D_{3}$ define toric singularities of type $T(1,11,3), T(1,3,1)$, and $T(2,2,1)$, respectively, and

$$
K_{X}^{2}=K_{M}^{2}+\delta(1,11,3)+\delta(1,3,1)+\delta(2,2,1)=-7+5+2+1=1 .
$$

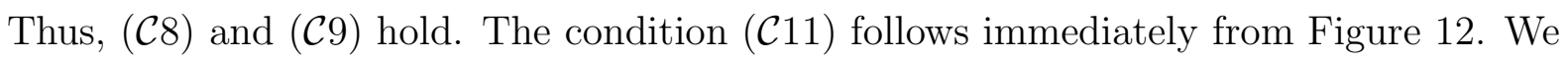

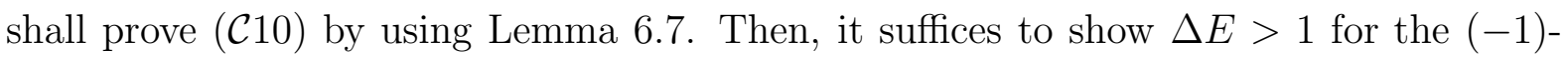
curve $E$ which joins the end (-4)-component of $D_{1}$ and the end (-2)-component of $D_{2}$. 
By Tables 1 and 2, we have

$$
\Delta E=(1-3 / 11)+(1-2 / 3)>1
$$

Hence, $(\underline{C} 10)$ follows. We shall prove the simply connectedness of $M \backslash D$ in case $\mathbb{k}=\mathbb{C}$ by applying Lemma 6.10.1. We have $\left(d_{1}, d_{2}, d_{3}\right)=(1,1,2)$ and $\left(n_{1}, n_{2}, n_{3}\right)=(11,3,2)$ for the type $T\left(d_{i}, n_{i}, a_{i}\right)$ of the singularity defined by $D_{i}$ for $1 \leq i \leq 3$. Looking at Figure 12 , we have the following $(-1)$-curves:

- the (-1)-curve $E$ which meets end components of $D_{1}$ and $D_{2}$.

- a $(-1)$-curve meeting the end (-4)-component of $D_{1}$ and an end (-3)-component of $D_{3}$.

Since $\operatorname{gcd}\left(d_{1} n_{1}, d_{2} n_{2}\right)=\operatorname{gcd}(11,3)=1$ and $\operatorname{gcd}\left(d_{1} n_{1}, d_{3} n_{3}\right)=\operatorname{gcd}(11,4)=1$, the conditions of Lemma 6.10.1 are all satisfied, and hence $M \backslash D$ is simply connected. Therefore, we have done all the tasks.

Example 7.7. Assume that $\operatorname{char}(\mathbb{k}) \neq 3$ and we set $K^{2}=3$. We consider the same cubic pencil $\Phi$ as in Example 7.6. We also consider the same $\bar{F}=\bar{F}_{1}+\bar{F}_{2}$, but define

$$
\bar{S}:=\bar{S}_{1}+N^{\sim} \quad \text { and } \quad \bar{G}:=\bar{G}^{+}-L_{1}^{\sim}=\sum_{1 \leq i \neq 3 \leq 9} \Gamma_{i} .
$$

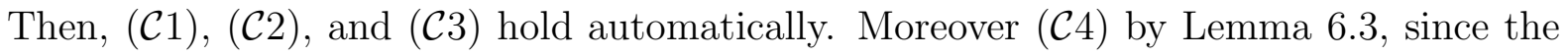
dual graph of $\bar{G}$ is $\mathrm{A}_{8}$ and $\operatorname{det}\left(\bar{G}_{i} \bar{G}_{j}\right) \not \equiv 0 \bmod \operatorname{char}(\mathbb{k})$. We have (A22) immediately from

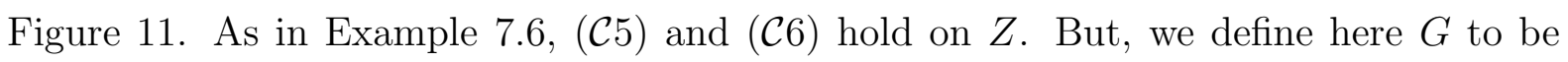
$S+F+J_{1}+J_{2}$. Let $\varphi: M \rightarrow Z$ be the birational morphism such that the total transform $B_{M}^{+}$of $\bar{B}^{+}$is as in Figure 13. Then, $\rho(M)=\rho(Z)+15=27$ and $K_{M}^{2}=-17$. Here,

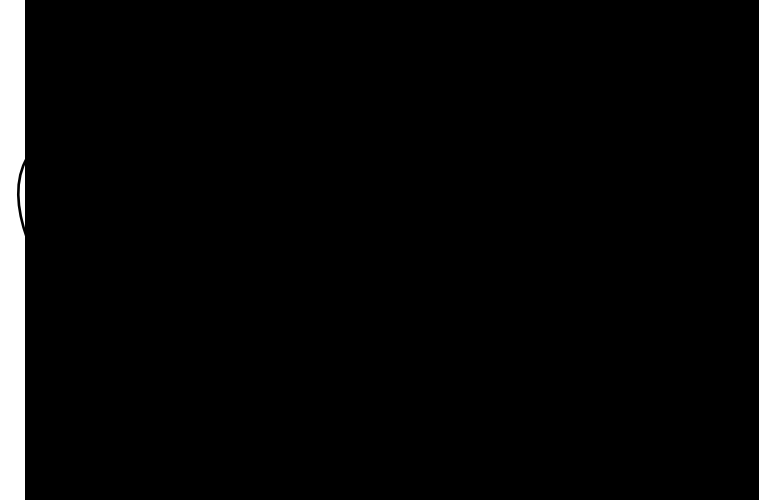

Figure 13. The rational surface $M$ in Example 7.7

we have a disjoint union $D$ of the following three linear chains of smooth rational curves satisfying (그):

$$
D_{1}=(2,7,7,2,2,3,2,2,2,2,3) \quad D_{2}=(5,3,2,2), \quad D_{3}=(9,2,2,2,2,2) .
$$


By Tables 1 and $2, D_{1}, D_{2}$, and $D_{3}$ define the toric singularities of type $T(1,63,34)$, $T(2,4,1)$, and $T(1,7,1)$, respectively, and

$$
K_{X}^{2}=K_{M}^{2}+\delta(1,63,34)+\delta(2,4,1)+\delta(1,7,1)=-17+11+3+6=3 .
$$

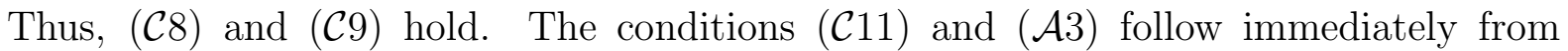
Figure 13. We shall prove (담 $)$ by using Lemma 6.7. Then, it suffices to check $\Delta E_{i}>0$ for $i=1,2,3$ for the (-1)-curves $E_{1}, E_{2}$, and $E_{3}$ on $M$ characterized by:

- $E_{1}$ joins the end $(-2)$-component of $D_{2}$ and the $(-9)$-curve in $D_{3}$.

- $E_{2}$ joins the end (-2)-component of $D_{3}$ and the third component of $D_{1}$ which is a $(-7)$-curve.

- $E_{3}$ joins the end $(-2)$-component of $D_{1}$ and the second component of $D_{1}$ which is a $(-7)$-curve.

By Tables 1 and 2, we can calculate

$$
\begin{gathered}
\Delta E_{1}=(1-3 / 4)+(1-1 / 7)>1, \quad \Delta E_{2}=(1-6 / 7)+(1-1 / 63)>1, \\
\Delta E_{3}=(1-34 / 63)+(1-5 / 63)>1 .
\end{gathered}
$$

Hence, (C10) holds. The ampleness of $K_{X}$ follows from (A22) and (A33) by Proposition 6.6.(4). We shall show that $M \backslash D$ is simply connected when $\mathbb{k}=\mathbb{C}$ by applying Lemma 6.10.1. We have $\left(d_{1}, d_{2}, d_{3}\right)=(1,2,1)$ and $\left(n_{1}, n_{2}, n_{3}\right)=(63,4,7)$ for the type $T\left(d_{i}, n_{i}, a_{i}\right)$ of the singularity defined by $D_{i}$ for $1 \leq i \leq 3$. Looking at Figure 13 , we have the following two $(-1)$-curves:

- the (-1)-curve $E_{1}$ which meets end components of $D_{2}$ and $D_{3}$.

- a $(-1)$-curve meeting the end (-3)-component of $D_{1}$ and the end (-5)-component of $D_{2}$.

Since $\operatorname{gcd}\left(d_{2} n_{2}, d_{3} n_{3}\right)=\operatorname{gcd}(8,7)=1$ and $\operatorname{gcd}\left(d_{1} n_{1}, d_{2} n_{2}\right)=\operatorname{gcd}(63,8)=1$, the conditions of Lemma 6.10.1 are all satisfied, and hence $M \backslash D$ is simply connected. Therefore, we have done all the tasks.

Example 7.8. Assume that $\operatorname{char}(\mathbb{k}) \neq 3$ and we set $K^{2}=2$. We define

$$
\phi_{0}=\mathrm{x}^{3}+\mathrm{yz}(\mathrm{y}+\mathrm{z}) \text { and } \quad \phi_{\infty}=3 \mathrm{xyz}
$$

Then, $\Phi_{0}$ is a smooth cubic curve, and $\Phi_{\infty}=L_{1}+L_{2}+L_{3}$ for the lines $L_{1}=(\mathrm{x})_{0}, L_{2}=(\mathrm{y})_{0}$, and $L_{3}=(\mathbf{z})_{0}$. In particular, (드) holds. Here, note that $P_{3}=(0: 0: 1)=L_{1} \cap L_{2}$ and $P_{2}=(0: 1: 0)=L_{1} \cap L_{3}$ are inflection points of $\Phi_{0}$, and that $L_{2}$ and $L_{3}$ are the tangent lines of $\Phi_{0}$ at $P_{3}$ and $P_{2}$, respectively (cf. Figure 14). The condition (C2) also holds. In fact, for $c \neq 0$, the divisor $\Phi_{c}=\left(\phi_{0}+c \phi_{\infty}\right)_{0}$ is singular if and only if $c=-\omega^{i}$ for $i=0$, 1,2 , where $\omega$ is a primitive cubic root of 1 , and $\Phi_{-\omega^{i}}$ is a nodal rational curve with the 


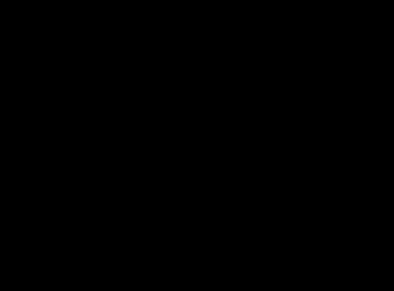

Figure 14. A pencil of cubics

node at $\left(1: \omega^{i}: \omega^{i}\right)$. We define $N$ to be the line passing through $(0: 1:-1) \in \Phi_{0} \cap L_{1}$ and the node $(1: 1: 1)$ of $\Phi_{-1}$. Thus, $N=(2 \mathrm{x}-\mathrm{y}-\mathrm{z})_{0}$ (cf. Figure 14). On the minimal elliptic fibration $\pi: Y \rightarrow \mathbb{P}_{\mathbb{k}}^{1}$ defined by $\Phi$, the configuration type of singular fibers is $\left(\mathrm{I}_{9}, \mathrm{I}_{1}, \mathrm{I}_{1}, \mathrm{I}_{1}\right)$, and the $(-1)$-curves exceptional for $Y \rightarrow \mathbb{P}_{\mathbb{k}}^{2}$ are mutually disjoint sections $\bar{S}_{1}, \bar{S}_{2}$, and $\bar{S}_{3}$ as in Figure [15, We may assume that $\bar{S}_{1}, \bar{S}_{2}, \bar{S}_{3}$ are contracted to the

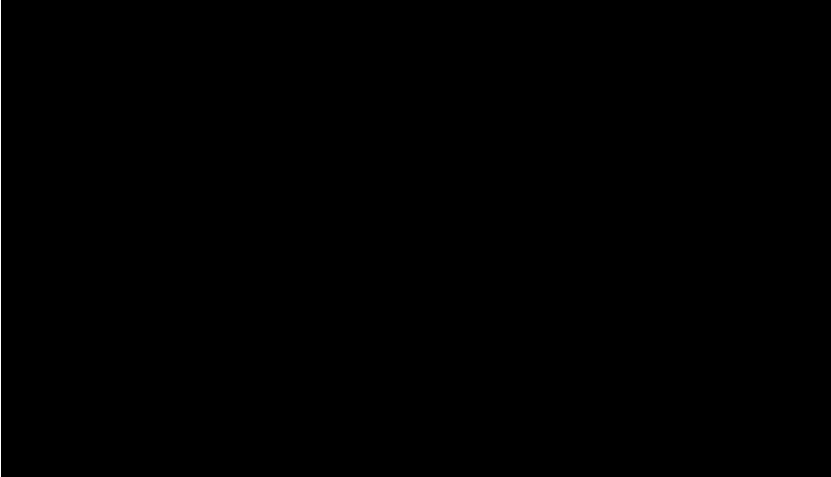

Figure 15. The rational elliptic surface $Y$ in Example 7.8

points $(0: 1:-1) \in \Phi_{0} \cap L_{1}, P_{3}=(0: 0: 1)$, and $P_{2}=(0: 1: 0)$, respectively. The three singular fibers of type $\mathrm{I}_{1}$ of $\pi$ are the proper transforms of $\Phi_{-\omega^{i}}$ for $i=0,1,2$. We set $\bar{F}_{1}$ and $\bar{F}_{2}$ to be the proper transforms of $\Phi_{-1}$ and $\Phi_{-\omega}$, respectively, and define $\bar{F}=\bar{F}_{1}+\bar{F}_{2}$. The irreducible components of the singular fiber of type $\mathrm{I}_{9}$ are labeled as $\Gamma_{0}+\Gamma_{1}+\cdots+\Gamma_{8}$ as a cyclic chain of rational curves in such a way that $\Gamma_{0}=L_{1}^{\sim}, \Gamma_{4}=L_{2}^{\sim}$, and $\Gamma_{5}=L_{3}^{\sim}$, where $L_{i}^{\sim}$ is the proper transform of $L_{i}$ in $Y$ for $i=1,2,3$. Then,

$$
\bar{S}_{1} \Gamma_{0}=\bar{S}_{2} \Gamma_{3}=\bar{S}_{2} \Gamma_{6}=1, \quad \text { and } \quad \bar{S}_{j} \Gamma_{i}=0
$$

for other $(i, j)$ with $0 \leq i \leq 8$ and $1 \leq j \leq 3$. The proper transform $N^{\sim}$ of $N$ in $Y$ is a bisection of $\pi$ with self-intersection number zero passing through the node of $\bar{F}_{1}$ but no nodes of the other singular fibers of type $\mathrm{I}_{1}$. We have

$$
N^{\sim} \bar{S}_{1}=N^{\sim} \Gamma_{4}=N^{\sim} \Gamma_{5}=1, \quad \text { and } \quad N^{\sim} \bar{S}_{j}=N^{\sim} \Gamma_{i}=0
$$

for $0 \leq i \neq 4,5 \leq 8$ and $j=2,3$ (cf. Figure 15). We define $\bar{S}:=\bar{S}_{3}+N^{\sim}$. Since the

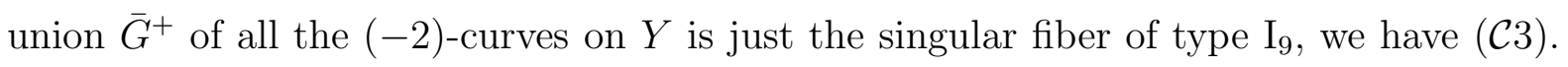


We define

$$
\bar{G}:=\bar{G}^{+}-\Gamma_{2}-\Gamma_{3}-\Gamma_{4}=\sum_{0 \leq i \neq 2,3,4 \leq 8} \Gamma_{i} .
$$

We shall show (ㄴ) without using Lemma 6.3. Assume that

$$
a_{1} \bar{S}_{3}+a_{2} N^{\sim}+\sum_{i=0}^{8} m_{i} \Gamma_{i} \sim p H
$$

for a Cartier divisor $H$, integers $a_{1}, a_{2}, m_{0}, \ldots, m_{8}$ with $m_{2}=m_{3}=m_{4}=0$, where $p=\operatorname{char}(\mathbb{k})$. Considering the intersection numbers with $\bar{S}_{1}, \bar{S}_{3}, N^{\sim}, \Gamma_{1}, \Gamma_{2}, \Gamma_{5}$, we have

$$
a_{2}+m_{0} \equiv-a_{1}+m_{6} \equiv m_{5} \equiv m_{0}-2 m_{1} \equiv m_{1} \equiv a_{2}-2 m_{5}+m_{6} \equiv 0 \quad \bmod p .
$$

Thus, $a_{1} \equiv a_{2} \equiv m_{i} \equiv 0 \bmod p$ for $0 \leq i \leq 6$. Moreover, we have $m_{7} \equiv m_{8} \equiv 0$ $\bmod p$ by considering the intersection numbers with $\Gamma_{0}$ and $\Gamma_{8}$. Hence, (담 holds. The condition (A22) fails. In fact, $\Gamma_{4} \subset Y \backslash(\bar{S} \cup \bar{G})$. Thus, it is impossible to require $K_{X}$ to be

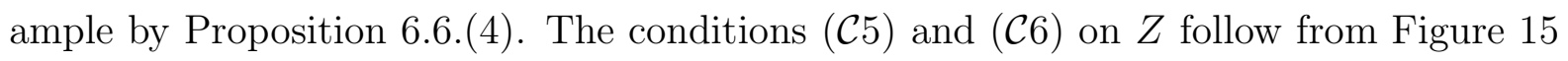
immediately. We define $B:=S+G+F+J_{1}$.

We take the birational morphism $\varphi: M \rightarrow Z$ so that the total transforms of $B+J_{2}$ and $\bar{G}^{+}$in $M$ form a configuration of curves as in Figure 16. Then, $\rho(M)=\rho(Z)+8=20$

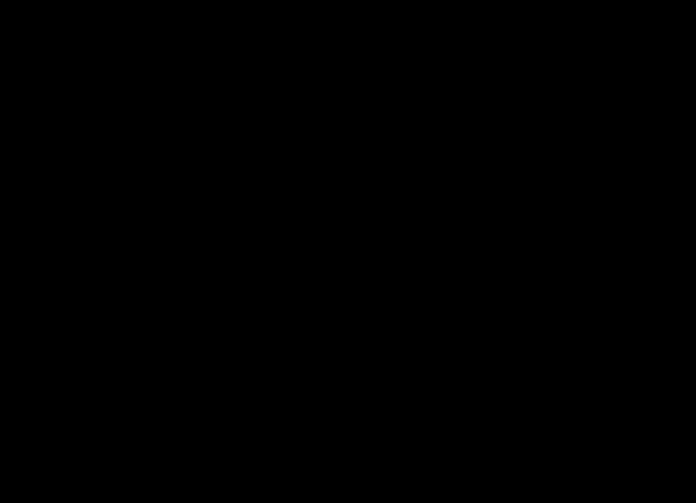

Figure 16. The rational surface $M$ in Example 7.8

and $K_{M}^{2}=-10$. Here, we have a disjoint union $D$ of the following two linear chains of smooth rational curves satisfying (드):

$$
D_{1}=\mathrm{LC}(6,5,2,3,2,3,2,2,2,2), \quad D_{2}=\mathrm{LC}(7,2,2,2) .
$$

By Tables 1 and 2, $D_{1}$ and $D_{2}$ define toric singularities of type $T(3,23,4)$ and $T(1,5,1)$, respectively, and

$$
K_{X}^{2}=K_{M}^{2}+\delta(3,23,4)+\delta(1,5,1)=-10+8+4=2 .
$$

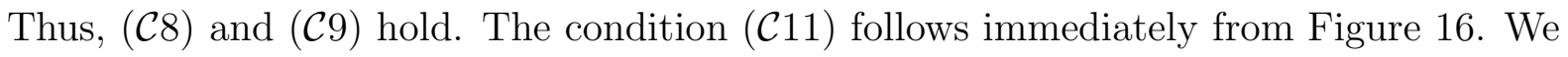

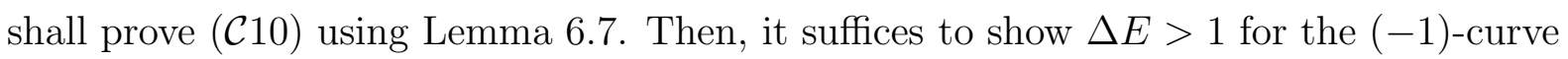


$E$ which joins the (-6)-curve of $D_{1}$ and the end (-2)-component of $D_{2}$. By Tables 1 and 2. we have

$$
\Delta E=(1-4 / 23)+(1-4 / 5)>1 .
$$

Hence, (다에 holds. We shall prove the simply connectedness of $M \backslash D$ in case $\mathbb{k}=\mathbb{C}$ by applying Lemma 6.10.1. We have $\left(d_{1}, d_{2}\right)=(3,1)$ and $\left(n_{1}, n_{2}\right)=(23,5)$ for the type $T\left(d_{i}, n_{i}, a_{i}\right)$ of the singularity defined by $D_{i}$ for $i=1,2$. Since the $(-1)$-curve $E$ meets end components of $D_{1}$ and $D_{2}$ and since $\operatorname{gcd}\left(d_{1} n_{1}, d_{2} n_{2}\right)=\operatorname{gcd}(3 \times 23,5)=1$, the conditions of Lemma 6.10.1 are satisfied, and hence $M \backslash D$ is simply connected. Therefore, we have done all the tasks.

Finally, we shall prove our main result. We restate the result.

Main Theorem. For any algebraically closed field $\mathbb{k}$ and for any integer $1 \leq K^{2} \leq 4$, there exists an algebraically simply connected minimal surface $\mathbb{S}$ of general type over $\mathbb{k}$ with $p_{g}(\mathbb{S})=q(\mathbb{S})=\operatorname{dim} \mathrm{H}^{2}\left(\mathbb{S}, \Theta_{\mathbb{S} / \mathbb{k}}\right)=0$ and $K_{\mathbb{S}}^{2}=K^{2}$ except $\left(\operatorname{char}(\mathbb{k}), K^{2}\right)=(2,4)$, where $\Theta_{\mathbb{S} / \mathbb{k}}$ denotes the tangent sheaf. Moreover, one can find such a surface with ample canonical divisor when $1 \leq K^{2} \leq 4$, except $\left(\operatorname{char}(\mathbb{k}), K^{2}\right)=(2,1),(2,2)$, and $(2,4)$.

Proof. Assume that $\left(p, K^{2}\right) \neq(2,4)$. Then, we have an algebraically simply connected minimal surface $\mathbb{S}$ of general type defined over $\mathbb{k}$ such that $p_{g}(\mathbb{S})=q(\mathbb{S})=\operatorname{dim} H^{2}\left(\mathbb{S}, \Theta_{\mathbb{S} / \mathbb{k}}\right)$ $=0$ and $K_{\mathbb{S}}^{2}=K^{2}$ by Propositions 6.8 and 6.11 applied to Examples 7.17 .8 above. In fact, the case $K^{2}=1$ is treated in Examples 7.3 (when $p \neq 2$ ) and 7.6 (when $p \neq 3$ ); the case $K^{2}=2$ in Examples 7.1 (when $p \neq 2,3$ ), 7.2 (when $p \neq 2$ ), and 7.8 (when $p \neq 3$ ); the case $K^{2}=3$ in Examples 7.4 (when $p \neq 2$ ) and 7.7 (when $p \neq 3$ ); and the case $K^{2}=4$ in Example 7.5 (when $p \neq 2$ ). Here, $K_{X}$ is not ample only in Examples 7.6 and 7.8. Thus, we can require $K_{\mathbb{S}}$ to be ample if $\left(p, K^{2}\right) \neq(2,1),(2,2)$. Hence, the proof has been completed.

\section{REFERENCES}

[EGA] A. Grothendieck, Éléments de géométrie algébrique (rédigés avec la collaboration de J. Dieudonné), Publ. Math. I.H.É.S., 4 (1960), 8 (1961), 11 (1961), 17 (1963), 20 (1964), 24 (1965), 28 (1966), 32 (1967).

[SGA1] A. Grothendieck et al., Revêtements Étales et Groupe Fondamental, Séminaire de Géométrie Algébrique du Bois Marie 1960-61 (SGA1), Lecture Notes in Math. 224, Springer-Verlag, 1971; A new updated edition: Documents Math. 3, Soc. Math. France, 2003.

[SGA2] A. Grothendieck et al., Cohomologie locale des faisceaux cohérents et théorèmes de Lefschetz locaux et globaux, Séminaire de Géométrie Algébrique du Bois Marie 1962 (SGA2), Adv. Stud. Pure Math. Vol. 2, North-Holland and Masson \& Cie, 1968; A new updated edition: Documents in Math. 4, Soc. Math. France, 2005.

[1] M. Artin, On isolated rational singularities on surfaces, Amer. J. of Math. 88 (1966), 129-136. 
[2] M. Artin, Algebraic approximation of structures over complete local rings, Publ. Math. I.H.É.S., 36 (1969), 23-58.

[3] M. Artin, Algebraization of formal moduli: I, Global Analysis, papers in honor of K. Kodaira (eds.

D. C. Spencer and S. Iyanaga), pp. 21-71, Univ. of Tokyo Press and Princeton Univ. Press, 1969.

[4] M. Artin, Versal deformations and algebraic stacks, Invent. Math. 27 (1974), 165-189.

[5] M. Artin, Algebraic construction of Brieskorn's resolutions, J. Algebra 29 (1974), 330-348.

[6] M. Artin, Coverings of the rational double points in characteristic $p$, Complex Analysis and Algebraic Geometry (W. L. Baily and T. Shioda eds.), pp. 11-22, Iwanami Shoten Publishers and Cambridge Univ. Press, 1977.

[7] R. Barlow, A simply connected surface of general type with $p_{g}=0$, Invent. Math. 79 (1984), 293-301.

[8] W. Barth, C. Peters and A. Van de Ven, Compact complex surfaces, Ergeb. Math. Grenzgeb. (3) 4, Springer-Verlag, Berlin, 1984.

[9] E. Brieskorn, Rationale Singularitäten komplexer Flächen, Invent. Math. 4 (1968), 336-358.

[10] D. Burns and J. Wahl, Local contributions to global deformations of surfaces, Invent. Math. 26 (1974), 67-88.

[11] V. I. Danilov, The geometry of toric varieties, Russ. Math. Surveys, 33:2 (1978), 97-154.

[12] M. Demazure, Sous-groupes algébriques de rang maximum du group de Cremona, Ann. Sci. Éc. Norm. Sup. $4^{e}$ série 3 (1970), 507-588.

[13] T. Ekedahl, Canonical models of surfaces of general type in positive characteristic, Publ. Math. I.H.É.S., 67 (1988), 97-144.

[14] R. Elkik, Solutions d'équations à coefficients dans un anneau hensélien, Ann. Sci. École Norm. Sup. (4) 6 (1973), 553-603.

[15] W. Fulton, Introduction to toric varieties, Ann. Math. Studies, 131, Princeton Univ. Press, 1993.

[16] D. Gieseker, Global moduli for surfaces of general type, Invent. Math. 43 (1977), 233-282.

[17] N. Hara, F-blowups of F-regular surface singularities, to appear in Proc. Amer. Math. Soc.

[18] R. Hartshorne, Algebraic Geometry, Grad. Texts in Math. 52, Springer-Verlag, 1977.

[19] F. Hirzebruch, Über vierdimensionale Riemannsche Flächen mehrdeutiger analytischer Funktionen von zwei komplexen Veränderlichen, Math. Ann. 126 (1953), 1-22.

[20] L. Illusie, Complexe Cotangent et Déformations I, Lecture Notes in Math. 239, Springer-Verlag, 1971.

[21] M.-N. Ishida and T. Oda, Torus embeddings and tangent complexes, Tohoku Math. J. 33 (1981), 337-381.

[22] T. Jarvis, W. Lang, G. Rimmasch, J. Rogers, E. Summers, and N. Petrosyan, Classification of singular fibers on rational elliptic surfaces in characteristic three, Comm. Algebra 33 (2005), 45334566.

[23] H. W. E. Jung, Darstellung der Funktionen eines algebraischen Körpers zweier unabhängigen Veränderlichen $x, y$ in der Umgebung einer Stelle $x=a, y=b$, J. reine angew. Math. 133 (1908), 289-314.

[24] G. Kempf, F. Knudsen, D. Mumford, and B. Saint-Donat, Toroidal embeddings. I, Lect. Notes in Math. 339, Springer-Verlag, 1973.

[25] K. Kodaira, On compact analytic surfaces. II, Ann. Math. 77 (1963), 563-626. 
[26] J. Kollár and N. I. Shepherd-Barron, Threefolds and deformations of surface singularities, Invent. Math. 91 (1988), 299-338.

[27] J. Kollár et. al., Flips and Abundance for algebraic threefolds, Astérisque 211, Soc. Math. France, 1992.

[28] W. Lang, Classical Godeaux surface in characteristic P, Math. Ann. 256 (1981), 419-427.

[29] W. Lang, Configurations of singular fibres on rational elliptic surfaces in characteristic two, Special issue in honor of Robin Hartshorne, Comm. Algebra 28 (2000), 5813-5836.

[30] H. B. Laufer, Deformations of resolutions of two-dimensional singularities, Complex analysis, 1972, Vol. I, Geometry of Singularities, Proc. Conf. Rice Univ. 1972, (H. L. Resnikoff and R. O. Wells, Jr. eds.), pp. 53-96, Rice Univ. Studies 59, no. 1, Rice Univ., Houston, 1973.

[31] H. B. Laufer, Taut two-dimensional singularities, Math. Ann. 205 (1973), 131-164.

[32] Y. Lee, Numerical bounds for degenerations of surfaces of general type, Internat. J. of Math. 10 (1999), 79-92.

[33] Y. Lee and J. Park, A simply connected surface of general type with $p_{g}=0$ and $K^{2}=2$, Invent. Math. 170 (2007), 483-505.

[34] S. Lichtenbaum and M. Schlessinger, The cotangent complex of a morphism, Trans. Amer. Math. Soc. 128 (1967), 41-70.

[35] C. Liedtke, Algebraic surfaces in positive characteristic, math.AG.arXiv:0912.4291.

[36] J. Lipman, Rational singularities with applications to algebraic surfaces and unique factorization, Publ. Math. I.H.É.S., 36 (1969), 195-279.

[37] E. Looijenga and J. Wahl, Quadratic functions and smoothing surface singularities, Topology, Vol. 25 (1986), 261-291.

[38] M. Manetti, Normal degenerations of the complex projective plane, J. reine angew. Math. 419 (1991), 89-118.

[39] N. Nakayama, Zariski-decomposition and Abundance, MSJ Memoirs 14, Math. Soc. Japan, 2004.

[40] N. Nakayama, Classification of log del Pezzo surfaces of index two, J. Math. Sci. Univ. Tokyo, 14 (2007), 293-498.

[41] T. Oda, Convex Bodies and Algebraic Geometry - An Introduction to the Theory of Toric Varieties, Ergebnisse der Math., (3) 15, Springer-Verlag, 1988.

[42] H. Park, J. Park, and D. Shin, A simply connected surface of general type with $p_{g}=0$ and $K^{2}=3$, Geom. Topol. 13 (2009), 743-767.

[43] H. Park, J. Park, and D. Shin, A simply connected surface of general type with $p_{g}=0$ and $K^{2}=4$, Geom. Topol. 13 (2009), 1483-1494.

[44] R. Rasdeaconu and I. Suvaina, Smooth structures and Einstein metrics on $\mathbb{C P}^{2} \# 5,6,7 \overline{\mathbb{C P}}^{2}$, Math. Proc. Cambridge Philos. Soc. 147 (2009), 409-417.

[45] M. Reid, Canonical 3-folds, Journées de Géometrie Algébrique d’Angers, Julliet 1979/Algebraic Geometry, Angers 1979 (ed. A. Beauville), pp. 273-310, Sijthoff and Noordhoff, 1980.

[46] M. Reid, Minimal models for canonical 3-folds, Algebraic Varieties and Analytic Varieties (ed. S. Iitaka), pp. 131-180, Adv. Stud. in Pure Math., 1 (1983), Kinokuniya and North-Holland.

[47] M. Reid, Young person's guide to canonical singularities, Algebraic Geometry, Bowdoin, 1985 (ed. S. Bloch), pp. 345-414, Proc. Sympo. in Pure Math. 46, Part 1, Amer. Math. Soc., 1987.

[48] D. S. Rim, Formal deformation theory, Groupes de Monodromie en Géométrie Algébrique, Séminaire de Géométrie Algébrique du Bois-Marie 1967-1969 (SGA7 I), dirigé par A. Grothendieck avec la 
collaboration de M. Raynaud et D. S. Rim, pp. 32-132 (Exposé VI), Lecture Notes in Math. 228, Springer-Verlag, 1972.

[49] M. Schlessinger, Functors of Artin rings, Trans. Amer. Math. Soc. 130 (1968), 208-222.

[50] N. I. Shepherd-Barron, Geography for surfaces of general type in positive characteristic, Invent. Math. 106 (1991), 263-274.

[51] N. I. Shepherd-Barron, Unstable vector bundles and linear systems on surfaces in characteristic $p$, Invent. Math. 106 (1991), 243-262.

[52] J. Stevens, On the versal deformation of cyclic quotient singularities, Singularity Theory and its Applications, Warwick 1989: Geometric Aspects of Singularities (D. Mond and J. Montaldi eds.), pp. 302-319, Lect. Notes in Math., 1462, Springer-Verlag, 1991.

[53] J. M. Wahl, Vanishing theorems for resolutions of surface singularities, Invent. Math. 31 (1975), $17-41$.

[54] J. M. Wahl, Elliptic deformations of minimally elliptic singularities, Math. Ann. 253 (1980), 241262.

[55] J. M. Wahl, Smoothings of normal surface singularities, Topology, Vol. 20 (1981), 219-246.

(Yongnam Lee) Department of Mathematics

Sogang University, Sinsu-dong, Mapo-gu, Seoul 121-742 Korea

E-mail address: ynlee@sogang.ac.kr

(Noboru Nakayama) Research Institute for Mathematical Sciences

Kyoto University, Kyoto 606-8502 JAPAN

E-mail address: nakayama@kurims.kyoto-u.ac.jp 


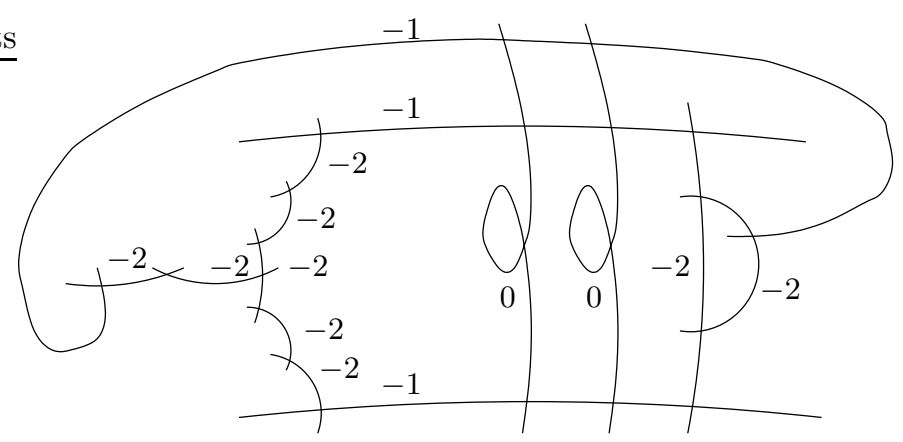

